University of Redlands

\title{
Testing Multivariate Alteration Detection for Tropical Deforestation using Synthetic Aperture Radar (SAR) and Drone Imagery in Mamoní Valley, Panama
}

A Major Individual Project submitted in partial satisfaction of the requirements for the degree of Master of Science in Geographic Information Systems

by

Abigail Bohman

Ruijin Ma, Ph.D., Committee Chair

Mark Kumler, Ph.D.

August 2019 
Testing Multivariate Alteration Detection for Tropical Deforestation using Synthetic Aperture Radar (SAR) and UAV Imagery in Mamoní Valley, Panama

Copyright (C) 2019

by

Abigail Bohman 
The report of Abigail Bohman is approved.

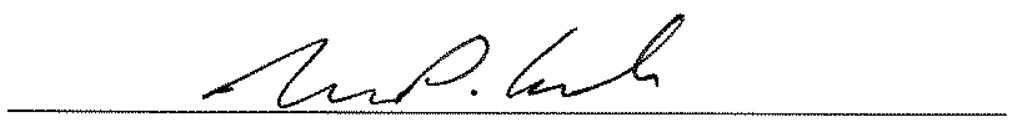

Mark Kumler, Ph.D.

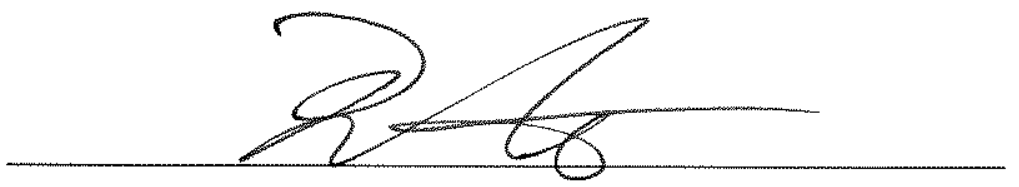

Ruijin Ma, Ph.D., Committee Chair

August 2019 



\section{Acknowledgements}

This was a challenging year in which I learned a lot about myself in addition to the topics that I explored for this project. Support from many people helped to push and carry me throughout the journey. First, I must express deep gratitude for my advisor, Ruijin $\mathrm{Ma}$, who was understanding and incredibly helpful throughout the process of my learning.

I must also thank my fellow members of Cohort 34 - I could not have done this year without you all! I am so grateful that we have gone through this all together, starting as complete strangers and ending as priceless friends. Another source of support and friendship came from Andrea Alvarado. Thank you, Andrea, for the conversation, advice, and endless amounts of work you do for this program. We appreciate you!

I also want to thank the Center for Spatial Studies faculty: Nate Strout, Dave Smith, and Steve Moore, as well as the Environmental Studies professor and project client: Dan Klooster. All of you have been my teachers and now I consider you friends. Thank you for your support ever since sophomore year of undergrad and continuing to be there during my graduate degree. I couldn't have done this without you, and I will miss you very much.

To my parents, Barb Maynes and Jeff Bohman, and my grandparents Betty and Alan Maynes: thank you for always being my foundation of support and inspiring me and encouraging me to make the world a better place, even when challenges arise.

Additionally, I must thank my older sister Meg Bohman Houbrick and my new brotherin-law, Brady Houbrick. Thank you for believing in me and being my biggest cheerleaders. You have supported me like only older siblings could.

Finally, I want to thank Hamish Henderson. Thank you for standing with me through all highs and lows, offering support, comfort, motivation, and irreplaceable advice. 



\begin{abstract}
Testing Multivariate Alteration Detection for Tropical Deforestation using Synthetic Aperture Radar (SAR) and Drone Imagery in Mamoní Valley, Panama

by

Abigail Bohman

Monitoring tropical deforestation poses a juxtaposition of urgency and difficulty. Because of nearly constant cloud cover in the tropics, heavily studied and widely implemented methods using multispectral satellite imagery to do large-scale land monitoring are not possible. Being an active sensing system that can penetrate through clouds, Synthetic Aperture Radar (SAR) solves this issue, making it one of the best opportunities for tropical forest change in the tropics.

In Mamoní Valley, Panama, Multivariate Alteration Detection (MAD) analysis was used with SAR Ground Range Detected imagery from the European Space Agency's (ESA) Sentinel 1 satellite to test its viability for detecting forest changes. Multispectral Unmanned Aerial Vehicle (UAV) imagery was used and tested for its viability to provide validation of the change detection analysis. Results of the methodology showed correlation between the SAR MAD results and various land cover types observed in the UAV imagery, though distinct multitemporal changes resulting from the analyses did not appear to correlate with changes shown in the UAV imagery. A comparison with Principal Component Analysis showed similar results, leading to the conclusion that the MAD method implemented was sound, but GRD SAR data may not be most suitable for this method. The results of the analyses can be used to focus future UAV mapping sites which may further develop the accuracy and implementation of SAR for tropical change detection.
\end{abstract}





\section{Table of Contents}

Chapter 1 - Introduction ................................................................................................. 1

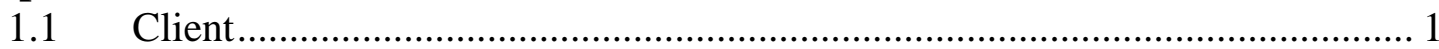

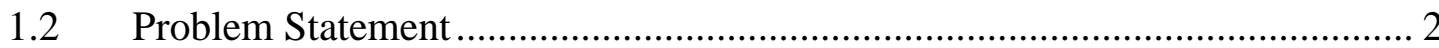

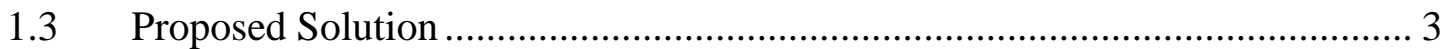

1.3.1 Goals and Objectives ............................................................................ 4

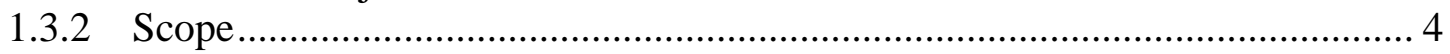

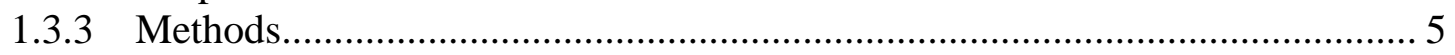

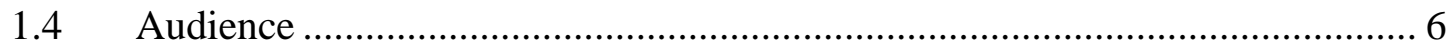

$1.5 \quad$ Overview of the Rest of this Report .............................................................. 6

Chapter 2 - Background and Literature Review ............................................................. 7

$2.1 \quad$ Tropical Deforestation Monitoring in Panama …………………………….... 7

2.2 GIS and Remote Sensing of the Environment ……….................................. 10

2.3 Change Detection Analyses with SAR Data................................................... 12

2.3.1 Multivariate Alteration Detection \& Maximum Autocorrelation Factor

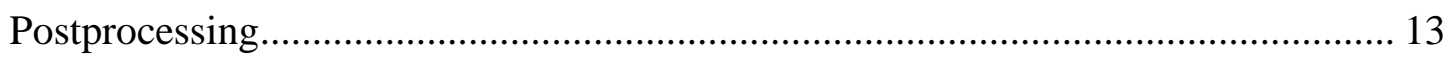

2.3.2 Principal Component Analysis ..................................................................... 14

2.3.3 Knowledge-Based Land Cover Classification \& Thematic Change................. 14

2.3.4 Coherent Change Detection Processing........................................................ 15

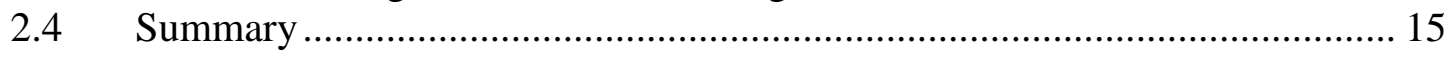

Chapter 3 - Systems Analysis and Design........................................................ 17

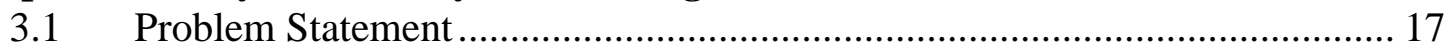

3.2 Requirements Analysis …………………………................................... 17

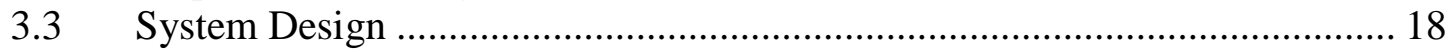

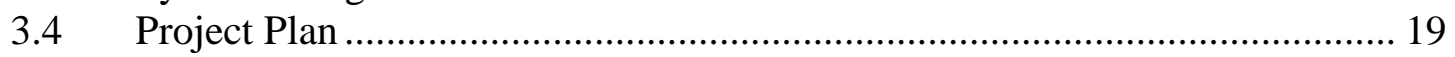

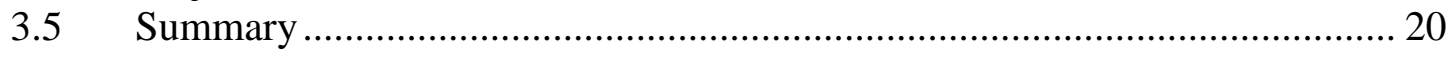

Chapter 4 - Database Design............................................................................... 21

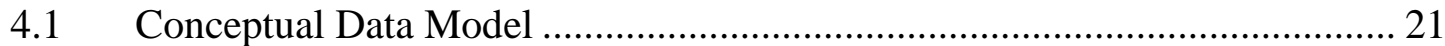

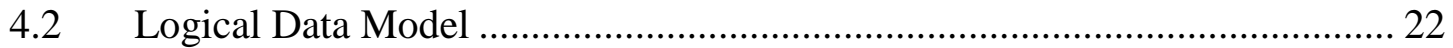

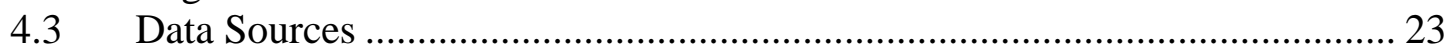

4.4 Data Collection Methods ..................................................................... 23

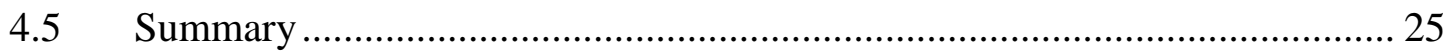

Chapter 5 - Implementation.......................................................................................... 27

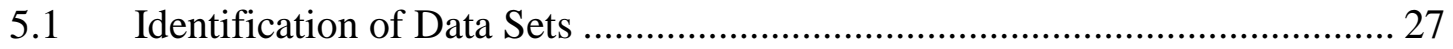

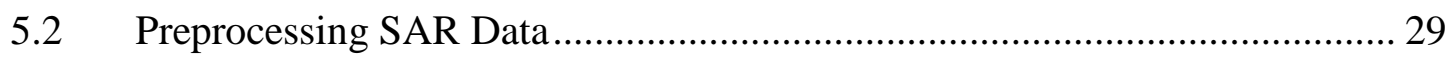

5.3 SAR Surface Change Detection Analysis ....................................................... 33

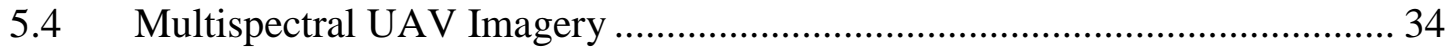

5.5 Automating Preprocessing and MAD Analysis ............................................. 39

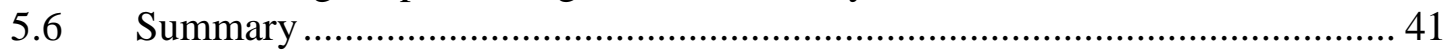

Chapter 6 - Results and Analysis...................................................................................... 43

6.1 MAD Results with UAV Control Areas ........................................................ 43

6.2 Change Detection with Principal Component Analysis ................................... 47 
6.2.1 Principal Component of VV Polarization .............................................. 48

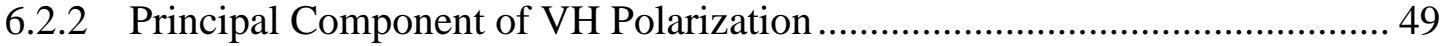

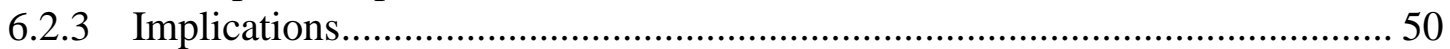

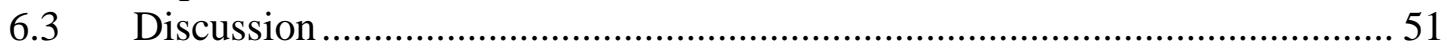

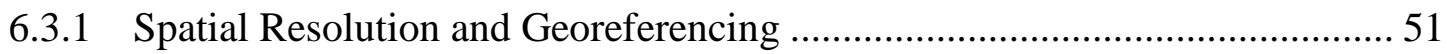

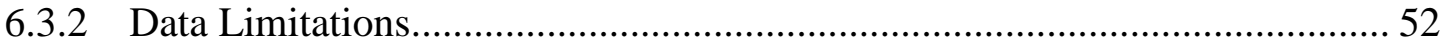

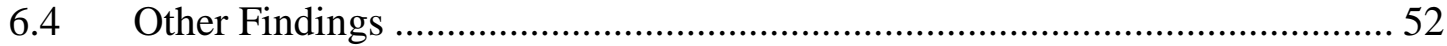

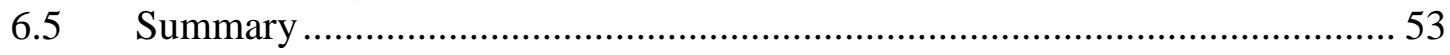

Chapter 7 - Conclusions and Future Work .......................................................... 55

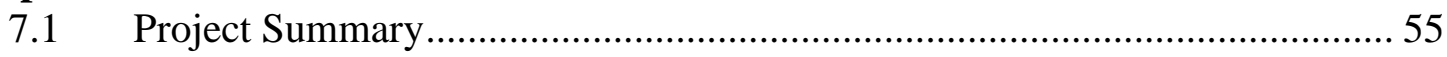

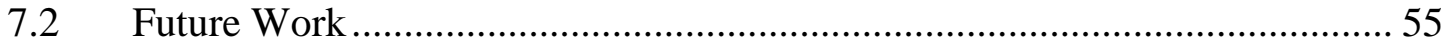

Works Cited ............................................................................................................................. 59

Appendix A. MAD Script in Python ................................................................................... 65

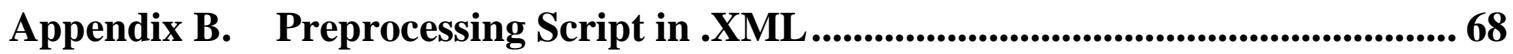




\section{Table of Figures}

Figure 1-1: Location of Mamoní Valley

Figure 1-2: Pasturelands in Mamoní Valley with a backdrop of primary rainforest. .. 3

Figure 2-1: Pasturelands where mature rainforest previously stood......................... 8

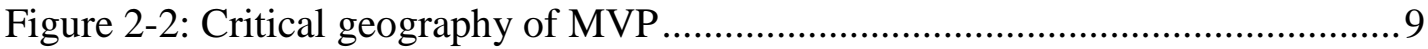

Figure 2-3: Vector data model versus raster data model ........................................ 11

Figure 2-4: Diagram of MAD, which uses Canonical Correlation Analysis............. 13

Figure 2-5: Principal Components visualized in a scatter plot ................................. 14

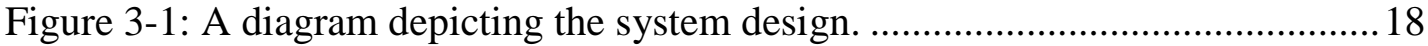

Figure 3-2: Original timeline in a Gantt Chart...................................................20

Figure 4-1: Diagram of Conceptual Model............................................................... 21

Figure 4-2: List of feature classes created and used for the project............................22

Figure 4-3: A Screenshot of the Copernicus Open Access Hub ................................2. 24

Figure 4-4: Snapshots from the Copernicus Open Access Hub...............................25

Figure 5-1: Drone imagery acquired from 2017 - 2019...........................................28

Figure 5-2: The approximate overlap depicted by orthomosaic boundaries..............29

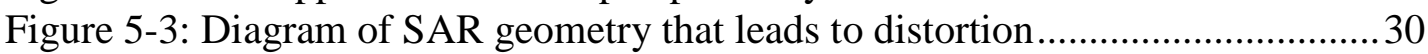

Figure 5-4: Before and after distortion correction in a city scene .............................. 31

Figure 5-5: Before and after terrain correction ................................................. 31

Figure 5-6: Before and after using the subset tool to focus on the study area........... 32

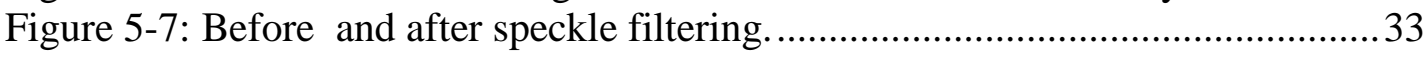

Figure 5-8: The original Ground Range Detected (GRD) images ................................ 34

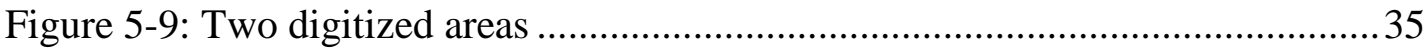

Figure 5-10: An instance of forest loss. 2018 UAV imagery cuts off in this area..... 36

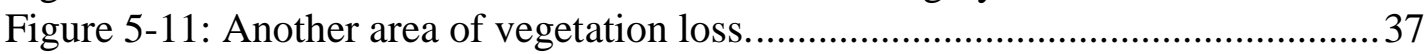

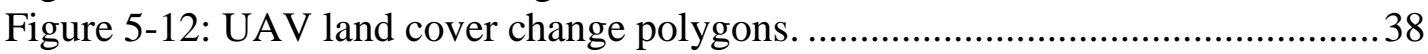

Figure 5-13: A diagram of the workflow used during project implementation......... 39

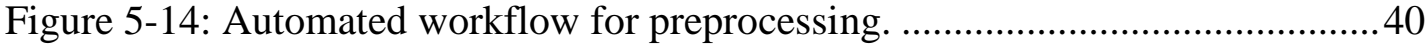

Figure 5-15: MAD script tool interface in ArcGIS Pro........................................... 41

Figure 6-1: Raw result of MAD Analysis................................................................ 43

Figure 6-2: Example of threshold applied to MAD result. ...................................... 44

Figure 6-3: Distribution of grouped pixel values...............................................45

Figure 6-4: A closer look at the thresholded MAD result with 2019 drone imagery.46

Figure 6-5: Scatter plot depicting principal components.................................... 47

Figure 6-6: First layer of PCA result. .................................................................... 48

Figure 6-7: A comparison of the second VV PC and MAD .................................49

Figure 6-8: Second Principal Components of PCA Analysis ....................................50

Figure 6-9: Inconsistency of georeferenced drone imagery. ...................................51

Figure 6-10: Overall pattern of Mamoní Valley .....................................................53

Figure 7-1: MAD result symbolized to show clustering of high and low values. .....56 



\section{List of Tables}

Table 3-2: Requirements and corresponding deliverables for the project. ...................... 18 



\section{List of Acronyms and Definitions}

$\begin{array}{ll}\text { AVHRR } & \text { Advanced Very High-Resolution Radar } \\ \text { COO } & \text { Chief Operating Officer } \\ \text { EC } & \text { European Commission } \\ \text { ESA } & \text { European Space Agency } \\ \text { EW } & \text { Extra-Wide Swath Mode } \\ \text { GIS } & \text { Geographic Information Systems } \\ \text { GPS } & \text { Global Positioning System } \\ \text { GRD } & \text { Ground Range Detected } \\ \text { IDE } & \text { Integrated Development Environment } \\ \text { IW } & \text { Interferometric Wide Swath Mode } \\ \text { NDVI } & \text { Normalized Difference Vegetation Index } \\ \text { NRT } & \text { Near-Real Time } \\ \text { MAD } & \text { Multivariate Alteration Detection } \\ \text { MAF } & \text { Maximum Autocorrelation Factor } \\ \text { MODIS } & \text { Moderate Resolution Imaging Spectroradiometer } \\ \text { MVP } & \text { Mamoní Valley Preserve } \\ \text { PC } & \text { Principal Component } \\ \text { PCA } & \text { Principal Component Analysis } \\ \text { SAR } & \text { Synthetic Aperture Radar } \\ \text { SLC } & \text { Single Look Complex } \\ \text { SM } & \text { Strip Map Mode } \\ \text { SNAP } & \text { SentiNel Applications Platform } \\ \text { UAV } & \text { Unmanned Aerial Vehicle } \\ \text { WV } & \text { Wave Mode }\end{array}$





\section{Chapter 1 - Introduction}

Think globally; act locally. This is a statement often shared to attest the need for autonomic and small-scale effort to deal with the causes and effects of climate change. In many countries, where impacts are disproportionate to the contribution of climate change, resources that would aid in this autonomic handling of climate issues are scarce. In the following sections, research will show how a tool is needed for viable and rapid monitoring of deforestation in tropical rainforests. The impacts of this land use practice go beyond the boundaries of the rainforest, and the stewards of the land are often small, indigenous populations. Geographic Information System (GIS) tools are the cutting-edge resources that would allow impactful autonomous stewardship and conservation. This project's purpose was to create a specialized tool and intuitive methodology that could be used by audiences with varying familiarity with GIS and spatial analyses.

\subsection{Client}

Claus Kjaerby is the Chief Operating Officer (COO) of the environmental conservation and leadership nonprofit organization, Geoversity. His background specializes in international indigenous and human rights, sustainable development and natural resource management. As stated by the organization, "Geoversity is an ecosystem of leaders and organizations collaborating in the pursuit of breakthroughs in human design, enterprise, and creative expression, inspired by Nature" (Geoversity, 2019). This ecosystem of founding entities includes the Center for Spatial Studies at the University of Redlands as well as other partners in forestry, agroeconomics and education around the world. As $\mathrm{COO}, \mathrm{Kjaerby}$ had the goal to understand past and present trends of land cover change in the organization's main conservation area: Mamoní Valley Preserve (MVP) in Central Panama, shown in Figure 1-1.

Daniel Klooster, Ph.D., is a Professor of Environmental Science at the University of Redlands, also with a background in Latin American culture and environmental issues. Klooster began the now ongoing relationship between the University and Geoversity. He has led annual undergraduate environmental GIS expeditions to MVP since 2017, and now has a vision to continue building on the undergraduate research, collaboration and program thereof.

Together, Kjaerby and Klooster have acted as the clients for this project. While Kjaerby resides in Panama, Klooster has been the immediate point of contact. 


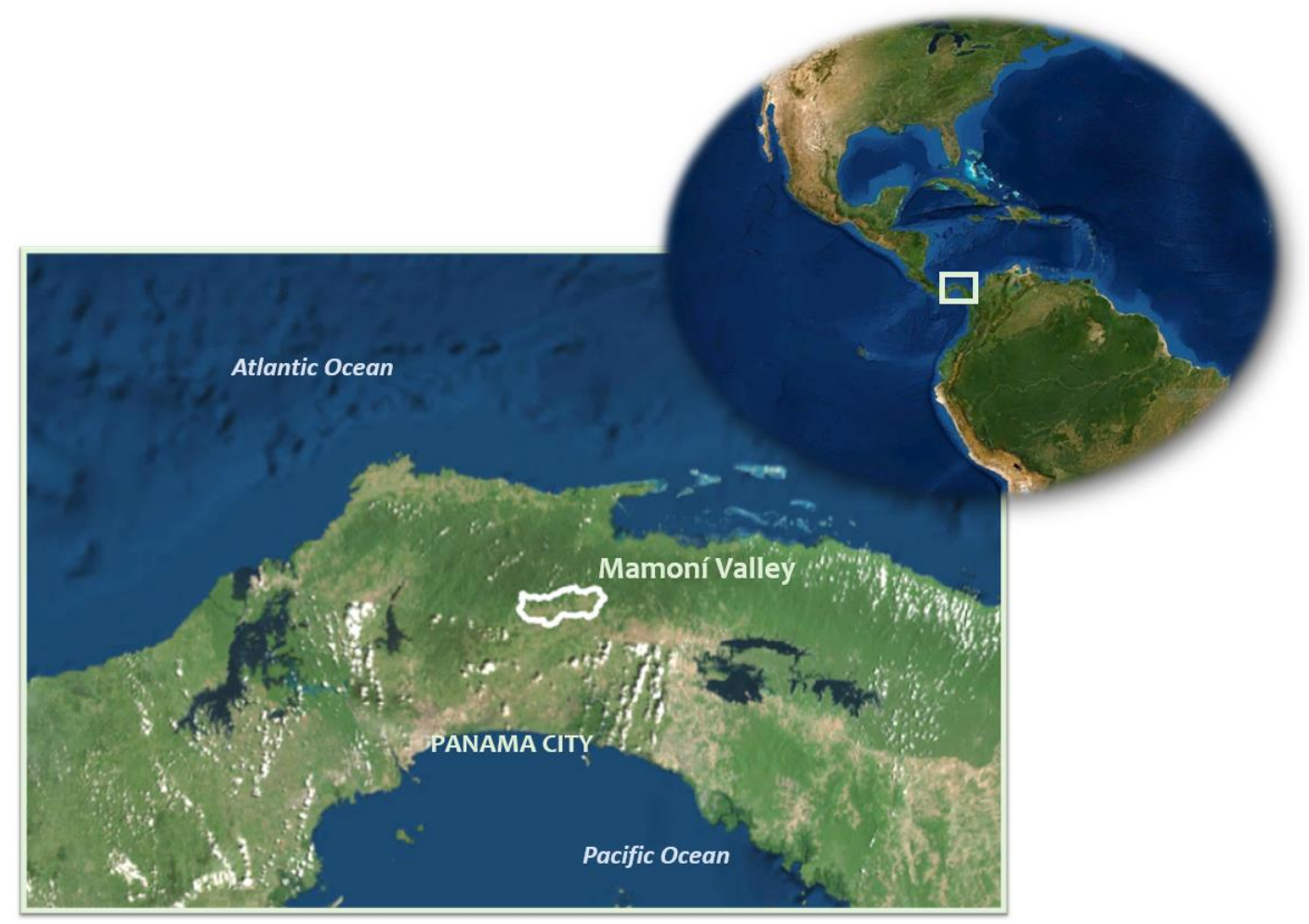

Figure 1-1: Location of Mamoní Valley.

\subsection{Problem Statement}

Tropical rainforests are one of the most biodiverse ecosystems on the planet and are being destroyed at unmonitored rates at many regional levels, as seen in Figure 1-2. MVP and Geoversity needed an easy-to-use tool that would allow non-GIS professionals to conduct forest change detection. 


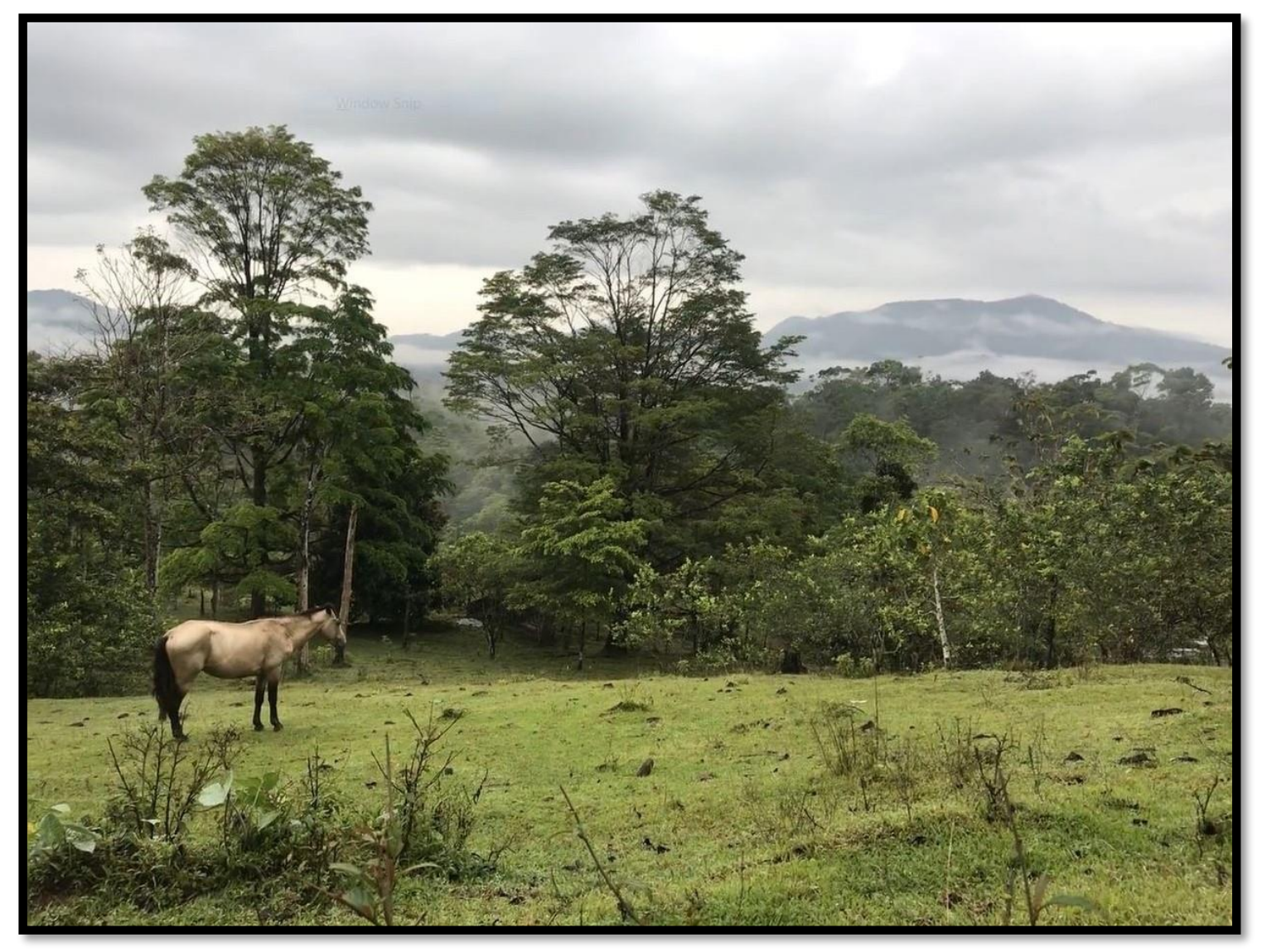

Figure 1-2: Pasturelands in Mamoní Valley with a backdrop of primary rainforest.

MVP was established to work against deforestation. The land was bought in the early 2000's to protect ancient rainforest areas and restore previously burned and developed areas within the Mamoní Valley Watershed. Years later, MVP management and stakeholders wanted to know what, if any, change had occurred to the land under their financial and physical efforts. However, being a small organization without designated personnel, monitoring forest dynamics had been an obstacle. The organization was not equipped with the resources and background to perform extensive statistical geographic analyses of forest change between dates. A simple methodology was needed so that it could be implemented by conservation area staff and neighboring communities facing similar difficulties.

\subsection{Proposed Solution}

Due to the rapid temporal change that is a characteristic of the humid tropics, conservation efforts require methods for rapid, repeatable land cover change analysis. Synthetic Aperture Radar (SAR) imagery was proposed as the primary data source due to its ability to penetrate through clouds and moisture which is often present in the tropics.

The European Space Agency's (ESA) Sentinel SAR data from the Copernicus Earth Observation Satellite Programme was used as input for a change detection image analysis 
in Esri's ArcGIS Pro interface. A workflow was created to guide preprocessing and automate analysis and post-processing of two images from separate dates being inspected to detect landcover change. Multispectral imagery collected using Unmanned Aerial Vehicles (UAV), also at times referred to as drones, at the same time over three years could be used as ground-truth data and to inform threshold values placed on the analysis results.

Finally, a written tutorial could guide non-GIS professionals in using the workflow and understanding the information it yields.

\subsubsection{Goals and Objectives}

The goal of this project was to help MVP monitor deforestation in its conservation area by creating and testing a methodology specific to the study area. The objectives were to modify and test a previously used statistical procedure to analyze change by creating a user-friendly interface and using ground-truth data to calibrate and validate the results of the analysis specifically to the study area.

These objectives were such so that the goal of continuous monitoring of the area could take place by preserve managers, students and researchers through using the methodology that is clear and informative about how and why it works.

Additionally, preserve managers and stakeholders are informed as they go forward in conservation efforts. Creating a methodology will provide a long-lasting way of monitoring the phenomena of deforestation and will continue to inform managers and policy makers in conservation as it provides a robust display of the urgency of finding sustainable land use practices. Together, these outcomes inform an argument and narrative that will be shared with communities and other stakeholders to bring awareness of the issues surrounding deforestation, climate change and sustainable development and agriculture.

\subsubsection{Scope}

The outputs of this project included static digital and paper landcover change maps, a Geodatabase and an automated workflow for interpretation of SAR data with integration of field observation. In order to complete these, a deep understanding of SAR sensors and SAR data needed to be acquired before moving forward to create an appropriate analytical workflow, as well as an accurate guide to use the workflow.

These outputs addressed all objectives of doing analysis: creating a methodology for long-term use and showing results in a clear way to non-scientific audiences. The resources required for these projects included the Esri software ArcGIS Pro and ArcGIS Online included in the enterprise account provided by the University of Redlands. The Python Wing Integrated Development Environment (IDE) Shell was used for writing source code for the main analysis tool, and a DJI Phantom 4 UAV along with a Garmin Global Positioning System (GPS) unit was used in the field to identify locations of various land cover types. In addition, the SentiNel Application Platform (SNAP) provided by the ESA Copernicus Program was used because it contains the specialized tools necessary for SAR data processing. 
Another aspect of the total scope was the educational portion of the project. This included full documentation of the workflow to be repeated in the future and initial trainings with project deployment.

\subsubsection{Methods}

In order to quantify change in the Mamoní Valley and display the results using a repeatable method, two images from different times were compared through a statistical image analysis.

SAR data was obtained from the ESA Copernicus Open Access Hub. Specifications such as orbit cycle and mode were given to ensure the compared images were compatible for analysis.

The two images from different dates were then preprocessed in SNAP with radar processing tools including geometric terrain correction and speckle filtering. This produced a single image with four bands which was then used as the input into an image processing script that was implemented in ArcGIS Pro as a script tool.

The analysis used is a statistical method called Multivariate Alteration Detection (MAD). MAD is a process which is based on canonical correlation analysis. Canonical correlation analysis finds the relationship between two groups of related variables, essentially finding the covariance within and between a set of variables. A linear combination of each group is produced, called a bivariate correlation or first canonical variates (Nielsen et al., 1998). This is the linear relationship of the variables within each group, and the correlation between the two separate linear relationships. Where canonical correlation is advantageous is in image processing events where there may be different sensors used to collect data, which means sets of data are not the same, such as using imagery from Landsat 8 and imagery from Sentinel 2. These sensors do not have uniform bands, however canonical correlation could be used because each set of data is individually represented by a linear combination, which is then correlated and ordered with linear combinations representing the second set of data.

Additionally, canonical correlation is advantageous when attempting to detect change. After the linear combinations from the two sets of data are created, they are placed into correlated pairs and ordered from most correlated to least correlated. During the MAD transformation step, the pairs of correlated linear combinations are subtracted, yielding an image showing maximum difference. Because corresponding pairs of canonical linear combinations are ordered by the similarity of each side of the pair, it is natural to base a change detection methodology on the differences between these pairs (Nielsen et al., 1998). This analysis yielded a raster that displayed pixel values indicating the magnitude of change between the two dates. More explanation about this approach is given in Section 2.3.1.

Spectral imagery was collected using UAVs, which served as ground-truthing data and informed proper thresholding values for the MAD result. By doing this, context was applied to the pixel values and the result could be classified to show land cover change. Finally, the workflows were documented and given to the client for continued autonomic use. 


\subsection{Audience}

The intended audience for this report includes GIS professionals and academics who are interested in learning about various applications of SAR imagery to GIS and remote sensing. The audience may also include individuals who are seeking an understanding of GIS and deforestation and how remote sensing can be used in the process. Finally, conservation area and other non-profit managers may use this project as a reference or material for generating ideas for potential practices that could enhance their programs.

\subsection{Overview of the Rest of this Report}

This report is divided into seven chapters. The second chapter provides contextual information of remote sensing, GIS, SAR data, and change detection. Chapter 3 discusses the project plan and system design including requirements and risk analysis. Chapter 4 goes over the database design of the project. Chapter 5 describes the implementation stage of the project. Chapter 6 includes a discussion of the results from project implementation and analysis. Finally, Chapter 7 reviews the entire project with a conclusion section and suggestions for additional work. 


\section{Chapter 2 - Background and Literature Review}

This chapter will provide background information and a review of pertinent literature in a series of topics that are embedded within this project. To provide a customized deforestation detection tool using Synthetic Aperture Radar (SAR) and to ensure accuracy and value, several topics need to be explored before project concept and design. The nature of SAR sensing is different from that of multispectral sensing, which has been used and documented much more frequently than the use of SAR in forest change detection. Because of this, the concepts and methods for SAR image analysis are explored. Additionally, the characteristics of forest and land cover change in the tropics need to be understood, so that a statistical change analysis can be given meaning through informed classification and thresholding.

\subsection{Tropical Deforestation Monitoring in Panama}

Beginning with its orogeny 4 million years ago, the isthmus of Panama has made a global impact on climate, biodiversity, and humans. Before the current physical geography of Panama, it was a peninsula extending south from North America, not yet connected to South America (Coates et al., 2003). The Pacific and Atlantic Oceans were part of the same oceanic system, exchanging cool and warm water locally within the tropics. Through tectonic shifting and plate subduction, the ocean between the Panamanian peninsula and what is now Colombia was filled with land, connecting the major continents of North and South America for the first time. This caused massive shifts in global oceanic circulation, which in turn influenced the climate around the globe. The "Great Biotic Exchange" took place, as plant and animal species that had evolved differently for millions of years began to coexist and mix in this new, extremely biodiverse, hotspot (Webb \& Stehli, 1985).

This intermixing of species is one of the reasons for Panama's exceptional biodiversity today. Its position in the tropics, where ample moisture and sun provide a never-ending energy source, is another reason. Plants and animals evolve faster here than in other climates, although they are not the only beings that are relevant to this land (Smith et al., 2012). The Panama Canal is tied to the western influence that has propelled much of the unchecked development of the country. Livelihoods surrounding pastoralism and agriculture were introduced and instilled upon native people who had lived in harmony with the ecosystem for years, leaving in its wake a trend to take rainforest and turn it into grassland for pasture. Increasing populations and western styles of livelihood demanded tangible goods, so massive trees made for an efficient wood harvesting market (Oestreicher et al., 2009). Many people still live in these remote parts of Panama, and the majority feel the pressure to turn forest into pasture, as seen in Figure 2-1. These are just a few of many social and environmental pressures that are present in the isthmus today. 


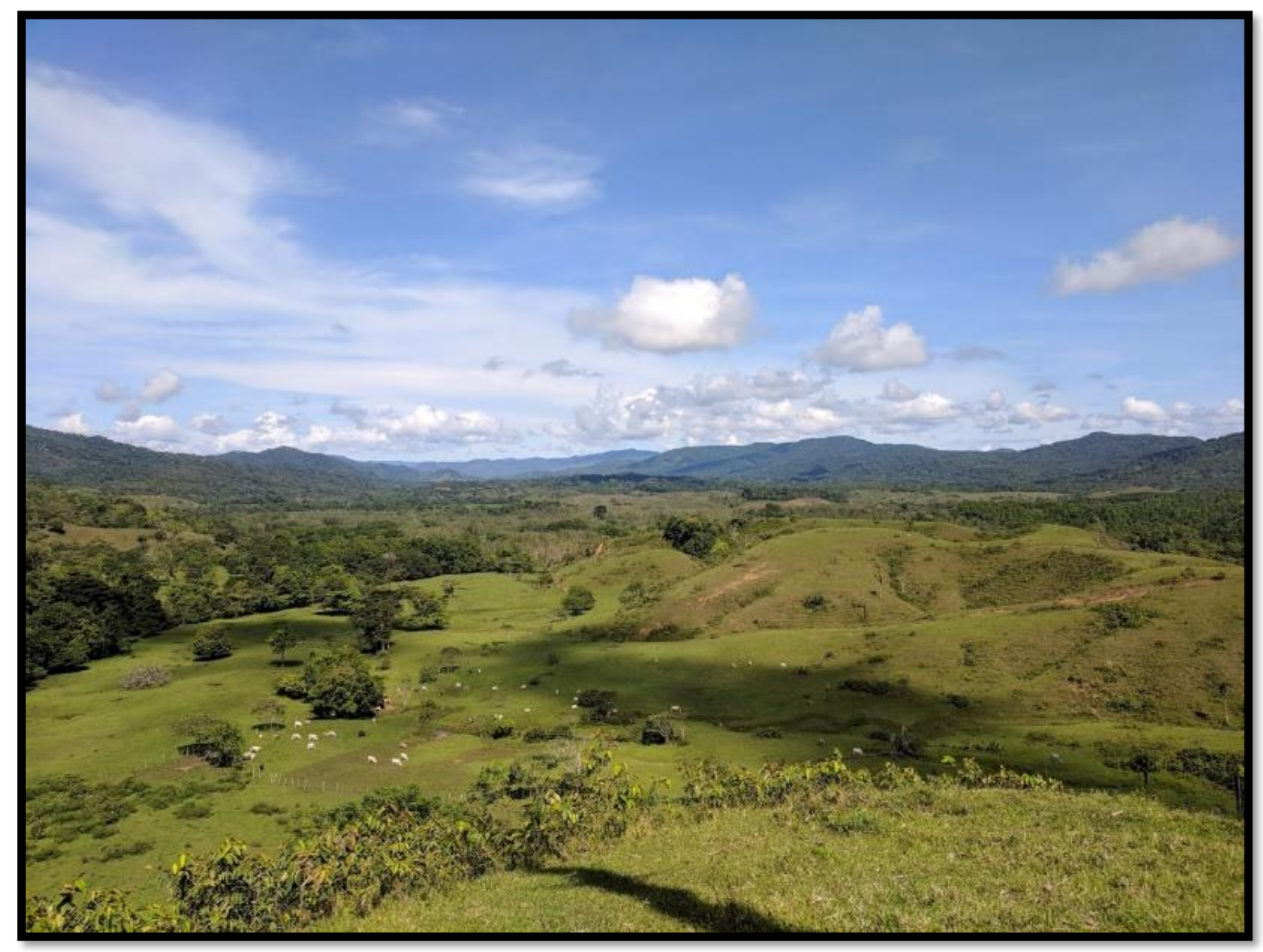

Figure 2-1: Pasturelands where mature rainforest previously stood.

The study area, Mamoní Valley Preserve (MVP) in the Mamoní River Watershed, is in the narrowest point of land in the Americas, connecting North and South America and separating the Pacific and Atlantic Oceans by only 50 kilometers. In its natural state, it is a corridor of primary rainforest and ecologically rich river valleys, where hundreds of species of migrating birds stop between the Americas. The MVP is within the ChocóDarién-Manabí eco-region, two hours east of Panama City along the continental divide as seen in Figure 2-2. The preserve shares the eastern border of Chagres National Park and the southwest border of Guna Yala, the semi-autonomous territory of the indigenous Gunas. As stated on the MVP website, "the preserve is a model of conservation and biocultural renewal within the 11,000-hectare upper watershed of the Rio Mamoní, one of the three largest rivers in the capital province of Panama" (Geoversity, 2019). In this region, there has been a trend of deforestation to expand room for agriculture, grazing and small development. These changes are typically carried out by local community members who live in the area but are slowly expanding their developed land in order to create livelihood from the local resources. This includes selective cutting of individual large trees, burning of smaller trees and shrubs, and slowly encroaching upon the edge of forested areas. In recent years, large-scale clear-cutting events have been increasingly 
less frequent. The smaller-scale changes happen more regularly, and are most of the changes that MVP wanted to monitor.

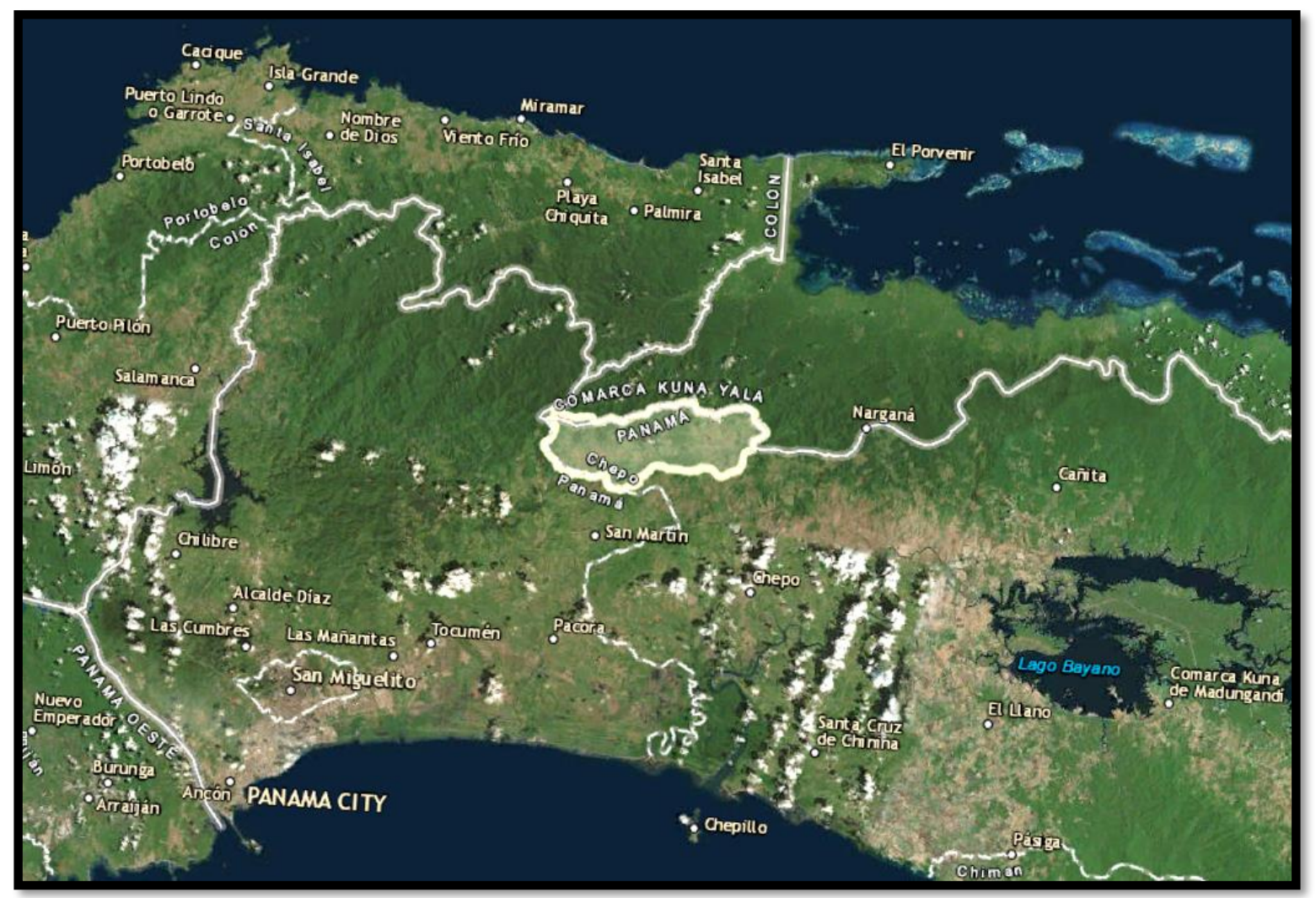

Figure 2-2: Critical geography of MVP.

Tropical rainforests are comprised of trees that can individually absorb at least 48 pounds of carbon dioxide annually (Rainforest Trust, 2017). Mature trees in tropical rainforests are thousands of years old, implying that thousands of years of carbon dioxide has been absorbed into the tree's biomass as the tree produces oxygen. In 2017, the Amazon Rainforest, one of the world's most noted rainforests, was recorded to produce approximately $20 \%$ of the entire world's oxygen supply (Rainforest Trust, 2017). As western ways of life spread to remote communities in tropical regions, the need for socalled developed styles of livelihood increases, demanding that more open space be produced for agriculture, farming, grazing and urban development. Common methods for acquiring this space are logging and slash-and-burn, both of which contribute to resource depletion, ecological disruption and global carbon dioxide levels rising.

The social and geographic boundary pressures present in Panama emphasize the need for GIS, where multiple complex factors may be brought together and analyzed in relation to each other. Spatial analysis can show how the resources present have been affected over time, make predictions for the future, and supplement plans for sustainable development.

Tropical forest monitoring with satellite imagery poses an interesting juxtaposition of necessity and difficulty. Globally, they are the largest forest biome, 
covering approximately $16 \%$ of the Earth's land surface (2.4 billion hectares) (Reiche et al., 2015; FAO 2011). Within this area, global cycles including the carbon, hydrological and biochemical cycles are regulated, and biodiversity, global economy, and local welfare are among the systems that benefit in a major way (Pan et al., 2011; Sasaki et al., 2011). Demand for tangible resources and climate change has increased pressure on these areas in the forms of deforestation and degradation (Achard et al., 2014 - B; Ghazoul \& Sheil 2010; Stern 2007). The problem has become so severe that tropical deforestation and degradation is the second largest source of greenhouse gases just after the combustion of fossil fuels (Reiche et al., 2015; van der Werf et al., 2009; Gullison et al., 2007).

This illustrates the great need for tropical forest monitoring on small and large scales which makes remote sensing a useful tool. Unfortunately, cloud cover (occurring $80 \%$ on yearly average) is ever-present in the tropics, making optical imagery, like that of Landsat, largely unusable (Souza et al., 2013; Herold 2009; Asner 2001). To address this, satellites with SAR capabilities, such as those in the European Space Agency's (ESA) Sentinel 1 system, are used as the long wavelength microwaves can penetrate through clouds (Lu et al., 2014; Souza et al., 2013; De Sy et al., 2012; Asner 2001; Malenovsky et al., 2012).

In addition to persistent cloud cover, other challenges surrounding satellite imagery and forest health are somewhat unique to the tropical rainforest. Unlike deforestation, forest degradation does not entirely remove the forest canopy. What occurs is a structural change of the forest, which is harder to see through remotely sensed imagery and often results in complex pixels that are difficult to analyze (Reiche et al., 2015, Achard et al., 2014 - B; Souza et al., 2005). Another challenge is that tropical ecosystems tend to change quickly with rapid canopy closure and understory regeneration once degradation occurs, though not necessarily with preferable species. As a result, changes that can be sensed by the remote sensing structures can disappear within 1-3 years (Souza et al., 2005; Asner et al., 2004). Advantageously, the twelve-day repeat cycle of Sentinel 1 helps to resolve that challenge.

\subsection{GIS and Remote Sensing of the Environment}

Many studies have shown the efficiency of remote sensing for large- and small-scale characterization and monitoring of land cover analyses. Remote sensing can provide a consistent and continuous spatial representation of large areas, and without the necessity of being directly in contact with the subject being studied, it is possible for rapid and large-scale data collection (Abdikan et al., 2016; Congalton et al., 2014, Friedl et al., 2010). Remotely sensed images can provide estimations of the areas of deforestation and degradation, called "activity data," while field observations can verify the degree of damage as well as carbon emission factors (Reiche et al,. 2015). When the two are used together, accurate land cover change can be estimated, and calculations can be made for specific carbon emissions from deforestation and degradation in a given area (Achard et al., 2014 - A).

In many cases, remotely sensed data is captured through imagery in various wavelengths of the spectral scale, efficiently acquiring high quantities of raw data. The image data, stored in a matrix (or raster) format is then readily available for processing 
and characterization with continuous spatial representation of large areas (Abdikan et al., 2016; Congalton et al., 2014, Friedl et al., 2010).

GIS allows automatic and organized analysis of geographic features and complex relationships between a seemingly limitless number of objects and subjects. Within these systems are two data models, vector and raster as seen in Figure 2-3 (Esri, n.d.). Vectors are used to represent discrete objects or features in the forms of points, lines and polygons, while rasters are used to represent continuous information or distributions such as land type, elevation or temperature (Esri, n.d.). In addition to the analysis-related pros of rasters, this data format is extremely beneficial in GIS because it will encompass very broad base characteristics and can be analyzed with vector data as well.

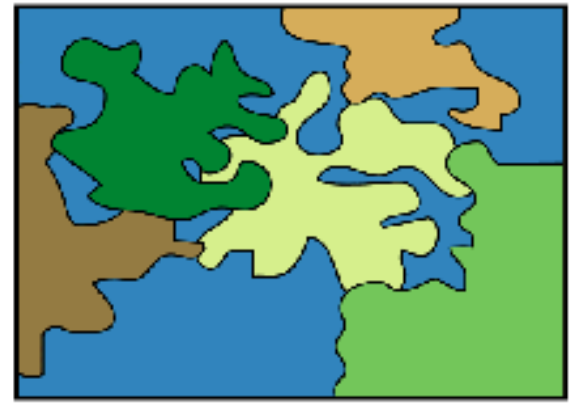

Polygon features

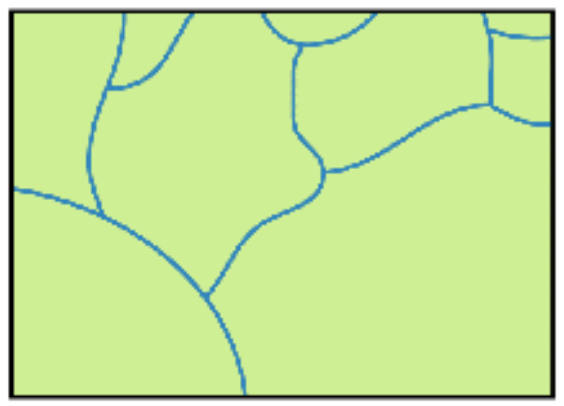

Line features

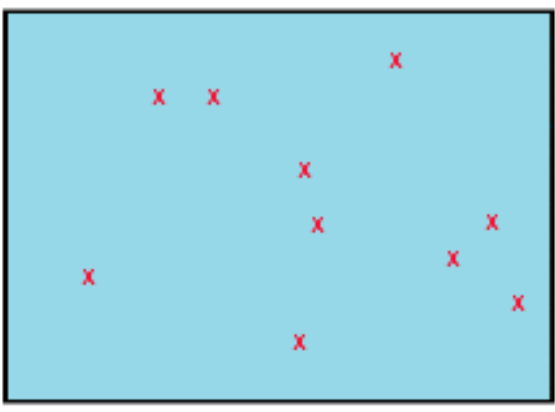

Point features

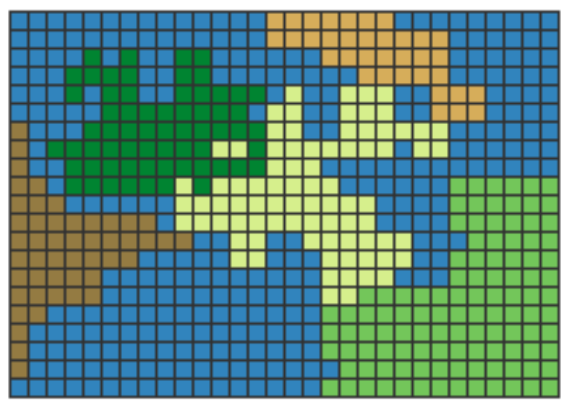

Raster polygon features

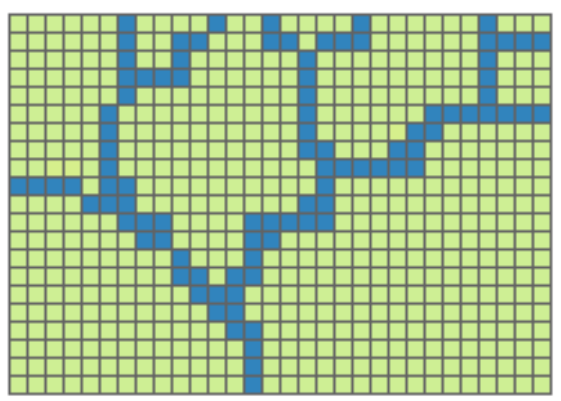

Raster line features

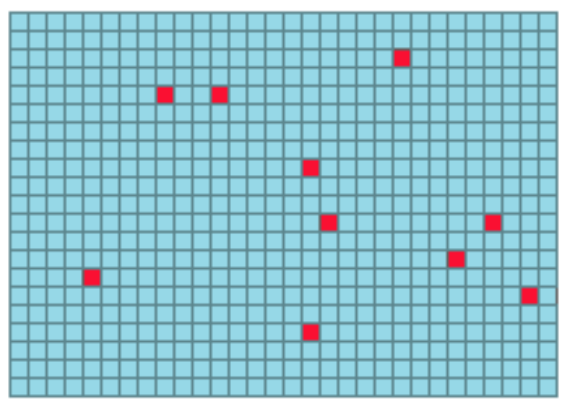

Raster point features

Figure 2-3: Vector data model versus raster data model (Esri, n.d.) 


\subsection{Change Detection Analyses with SAR Data}

The benefits of utilizing SAR image data are extensive, as its active-sensor approach allows it to be unaffected by many environmental characteristics that are necessary for successful optical remote-sensing. The active sensor transmits its own radar pulse and records the return, bypassing the need for an external energy source to record. The nature of the microwave radar beam $(.3-30 \mathrm{~cm})$ that is actively emitted is such that it can penetrate through atmospheric and lithospheric phenomena including clouds and rain (Reiche et al., 2015). Like multispectral images, SAR images are often used to detect changes over time, particularly in the case of satellite spacecraft which have continuous orbit cycles. Since 2015, SAR imagery from the ESA Sentinel 1 satellite sensor within the Copernicus mission has provided imagery that is freely available.

Phase and intensity are the types of data that SAR sensing systems collect, meaning that SAR processing methods must usually be different than typical optical image analyses, as spectral information is recorded and stored differently (European Space Agency Sentinel Online, n.d.). This calls for various statistical and conceptual approaches to detecting land cover change between two dates, implying two images. The following paragraphs will outline several SAR image data methods that have been studied for environmental change detection.

The Sentinel-1 mission is the European Radar Observatory for the Copernicus joint initiative of the European Commission (EC) and the ESA. The initiative of this mission is to implement information services concerning the environment and security. Included in the mission is C-band SAR operating in four modes with resolution as little as five meters and coverage up to $400 \mathrm{~km}$ (Attema et al., 2007). The modes mentioned are the Strip Map (SM), Interferometric Wide Swath (IW), Extra Wide Swath (EW), and Wave (WV) modes (European Space Agency, n.d.-A; European Space Agency, n.d.-B; Attema et al., 2007). Also included is dual polarization capability, a near-polar sunsynchronous orbit and a twelve-day repeat cycle (Attema et al., 2007). More on the missions can be found on the ESA Sentinel website. Many studies have shown how multi-sensor approaches combining SAR and optical sensors, such as those provided in the Sentinel system, have increased forest mapping accuracy (Reiche et al., 2015; Hoan et al., 2013; Lehmann et al., 2013; Vaglio Laurin et al., 2013; Lehmann et al., 2011; Walker et al., 2010; Erasmi \& Twele 2009; Almeida-Filho et al., 2007; Kuplich 2006; Rignot et al., 1997).

Previous remote sensing near-real-time (NRT) monitoring systems have relied on coarse resolution imagery such as a Moderate Resolution Imaging Spectroradiometer (MODIS) time series imagery to provide up-to-date information on deforested areas (Reiche et al., 2015; Wheeler et al., 2014; Hammer et al., 2009; Shimabukuro et al., 2006). However, these systems have spatial resolutions as low as 500 meters, causing small scale changes (such as those in Mamoní Valley) to be neither detected nor dealt with, showing a lack of medium or high-resolution satellite-based monitoring systems (Reiche et al., 2015; Hammer et al., 2014; Tyukavina et al., 2013). The use of SAR and optical time series methods for detecting forest changes has been minimal and leaves "lots of potential unexplored" (Lu et al., 2014; Reiche et al., 2014; Zhang 2010). 


\subsubsection{Multivariate Alteration Detection \& Maximum Autocorrelation Factor Postprocessing}

When using a Ground Range Detected (GRD) SAR product, information is given primarily in intensity. These kinds of analyses identify changes in the mean backscatter of a scene, which can be calculated from pixel intensity (Preiss \& Stacy, 2006). This works for change detection because the mean backscatter power would be constant over a region if it were an unvaried region. Therefore, if a discrepancy was present, an alteration to the mean backscatter would also be present.

Multivariate Alteration Detection (MAD) is a process which is based on canonical correlation analysis. Canonical correlation analysis creates linear combinations of two different sets of variables. The linear combination of each group is called a bivariate correlation and the two highest correlated are first canonical variates (Nielsen et al., 1998). This is the linear transformation of the variables within each group, and the correlation between the two separate linear transformations. Canonical correlation is advantageous when attempting to detect changes, as corresponding linear combinations will naturally show the difference between them when they are subtracted. This helps to identify features within a dataset that are important for explaining the covariation between sets of variables.

The final step of MAD finds the difference between each set of canonical variates, as depicted in Figure 2-4.

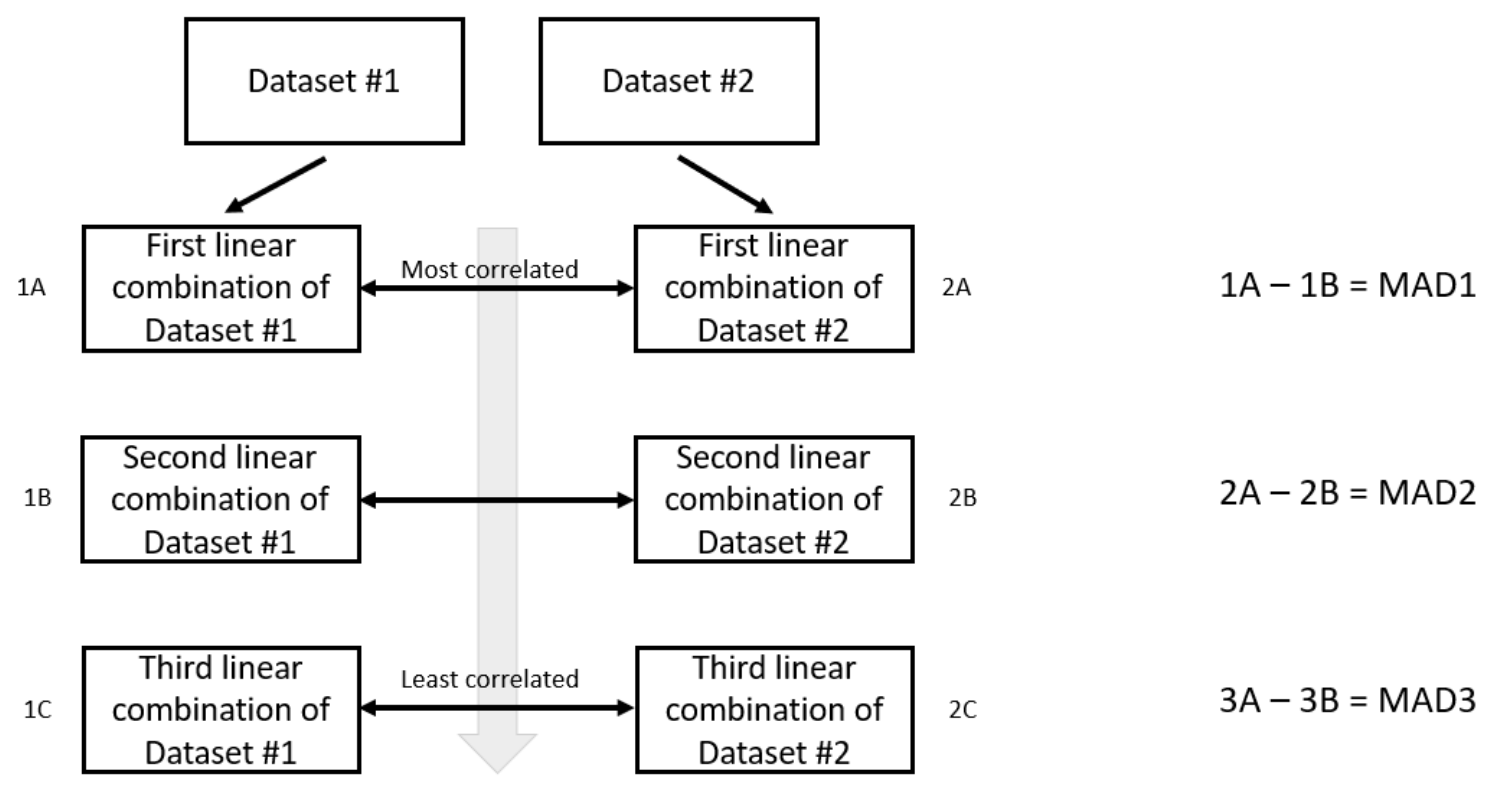

Figure 2-4: Diagram of MAD, which uses Canonical Correlation Analysis.

Maximum Autocorrelation Factor (MAF) transformation, is a potential last step which transforms the image in a way that is built upon the idea of principal component, where new variates are calculated to maximize the autocorrelation of each pixel. 
Essentially, this finds the maximum change between the linear combinations and groups them such that the autocorrelation of pixels is maximized (Nielsen et al., 1998).

\subsubsection{Principal Component Analysis}

Principal Component Analysis (PCA) aims to remove the correlation between different measurements such as image bands. It transforms the data to a new set of measurements that are independent and perpendicular to each other. The new measurements are called Principal components. PCA identifies new variables which are represented in linear combinations of the original variables (Ringnér, 2008). If the samples are plotted on a graph, the first principal component is a line which follows the trend and direction of the data, as shown in Figure 2-5. The second principal component goes perpendicular to the first one, forcing the original variables to fit into a set of values that does not have as much variation as the first principal component, also shown in Figure 2-5. This second principal component is commonly analyzed for change detection between two images as it shows where the original variables do not correlate.

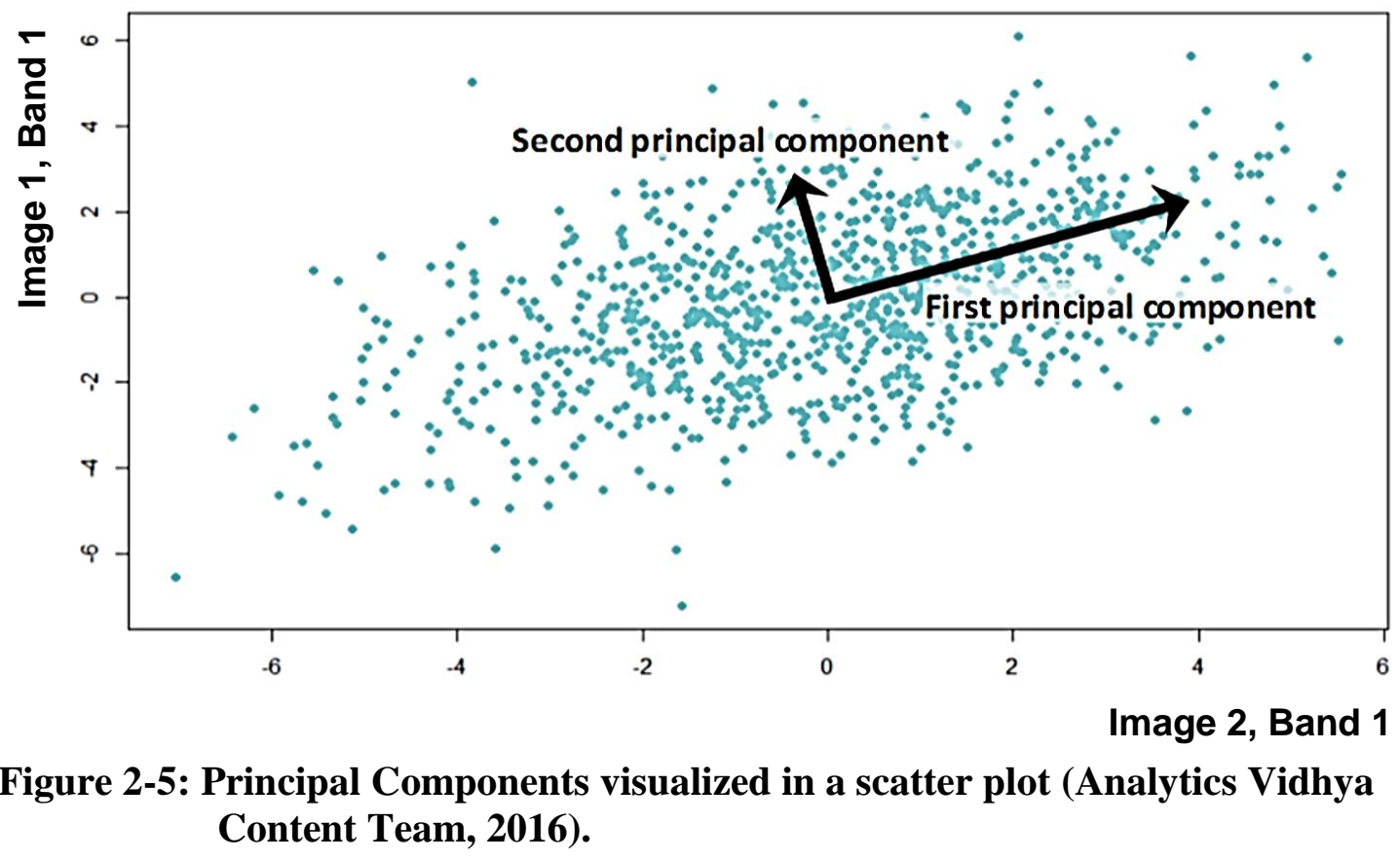

\subsubsection{Knowledge-Based Land Cover Classification \& Thematic Change}

This method aims to provide a clear correlation between terrain surfaces and sensor data. It is based on the idea of having a set of known variables, such as land cover classes, by which to compare with a hierarchically-derived classified SAR image (Dobson et al., 1996). In a sense, this is an expanded method of supervised classification.

The classifier can be designed to use the known nature of radar backscattering to construct thresholds in a sequential format. The classifier produces two levels of classification: the first differentiating terrain based on structure, into urban areas, short 
vegetation and tall vegetation. The second is a sub-classification that can differentiate between plant architectural properties and texture; woody stems and foliage (Dobson et al., 1996). In a study using this methodology, a test analysis showed that this SAR classifier yielded a higher rate of accuracy compared to an unsupervised classification method using multispectral Advanced Very High-Resolution Radar (AVHRR) data (Dobson et al., 1996).

To detect change using this method, the final step would be to perform raster calculations in which the change from one land cover class to another would be noted. A supplemental method to detect change over time is to employ a swipe or comparison tool of two classified images in which land cover can be visually interpreted.

\subsubsection{Coherent Change Detection Processing}

While a GRD SAR product provides only intensity information, a Single-Look Complex (SLC) SAR product contains two types of data: phase and intensity. The two numbers associated with those data types means that this SAR product uses a complex number. In change analyses with complex products, the correlation between images is given by a coefficient. The coefficient is what is commonly referred to as the "coherence." The coherence coefficient quantifies the extent to which the two data types are common between the two images. In other words, it allows for multitemporal images to be crosscorrelated and measured in terms of scene disturbance, given that all the appropriate preprocessing has been completed (Preiss \& Stacy, 2006). Using SLC data requires a more extensive processing procedure than that of GRD.

\subsection{Summary}

With a medium spatial resolution $(10-30 \mathrm{~m})$ and support from field observations for ground-truthing, satellite remote sensing is the most appropriate tool for assessing forest area change in most tropical countries (Reiche et al., 2015; De Sy et al., 2012; Achard et al., 2010;). Remotely sensed images can provide estimations of the areas of deforestation and degradation, called "activity data," while field observations can verify the degree of damage as well as carbon emission factors (Reiche et al., 2015). When the two are used together, accurate land cover change can be estimated, and calculations can be made for specific carbon emissions from deforestation and degradation in a given area (Achard et al., 2014). Going forward, these data components were integrated to provide an accurate, customized tool for tropical deforestation monitoring. 



\section{Chapter 3 - Systems Analysis and Design}

This chapter will discuss the concepts and design behind the project. This includes an exploration of functional and non-functional requirements and assessing how they will be used to inform the overall design of the project system and plan.

\subsection{Problem Statement}

Scientific analysis of tropical deforestation is needed in order to understand the issue and stimulate significant conservation efforts. While the problem affects many people, there are limits on the accessibility and understanding to advanced analytical procedures. This project aimed to simplify change detection imagery analyses for a specific study area and provide documentation for non-technical audiences to use and repeat the scientific methods independently.

\subsection{Requirements Analysis}

Some requirements were collected during consultations with the client. At these meetings, updates of work were given and new information that had come up was discussed. Upon the completion of the exchange of information, the requirements were updated.

Other requirements were collected as data processing took place, as a deeper understanding was built regarding the methods that were necessary in the project. This also led to a deeper sense of the scope and time constraints that were involved with fulfilling each functional requirement. With a better sense of the scope of the project, the requirements were added to and updated, which also tracked progress of defining and understanding requirements.

In order to reach the objectives of the client, the requirements for the project were broken down into the key components of analysis, ensuring accuracy, and user

functionalities, see Table 3.1. The analysis component needed to have the functionality of being an automatic analysis, including selecting the study area and preprocessing. This requirement came from the direct objectives of the client as it informs the user of the changes in land cover that are present in the study area.

Evaluating accuracy was a requirement built into the system which cross-checked the analysis results with several ground truthing checks.

The user functionalities of the system were further broken down into land change maps and succinct, understandable user guides. The maps included a further step of analysis that depicted the types of changes indicated but not identified from the image analysis. The functionality of the user guides was to be clear enough that non-scientific or non-GIS professionals could easily use the system. 
Table 3-1: Requirements and corresponding deliverables for the project.

\begin{tabular}{|c|c|}
\hline Deliverable & Requirement \\
\hline Change Detection Tool & $\begin{array}{l}\text { - Change detection based on multivariate } \\
\text { alternation detection } \\
\text { - Test results on drone imagery }\end{array}$ \\
\hline User Products & $\begin{array}{l}\text { - Land cover change maps that show } \\
\text { deforestation versus growth } \\
\text { - Automated change detection tool in ArcGIS } \\
\text { Pro } \\
\text { - Automated preprocessing tool }\end{array}$ \\
\hline
\end{tabular}

\subsection{System Design}

The major components of this project were a methodology for land cover change detection, user guides and field involvement, and a demonstration of the workflow which was a finalized land change map cross-checked with the methodology previously used. These components fit together as shown in Figure 3-1.

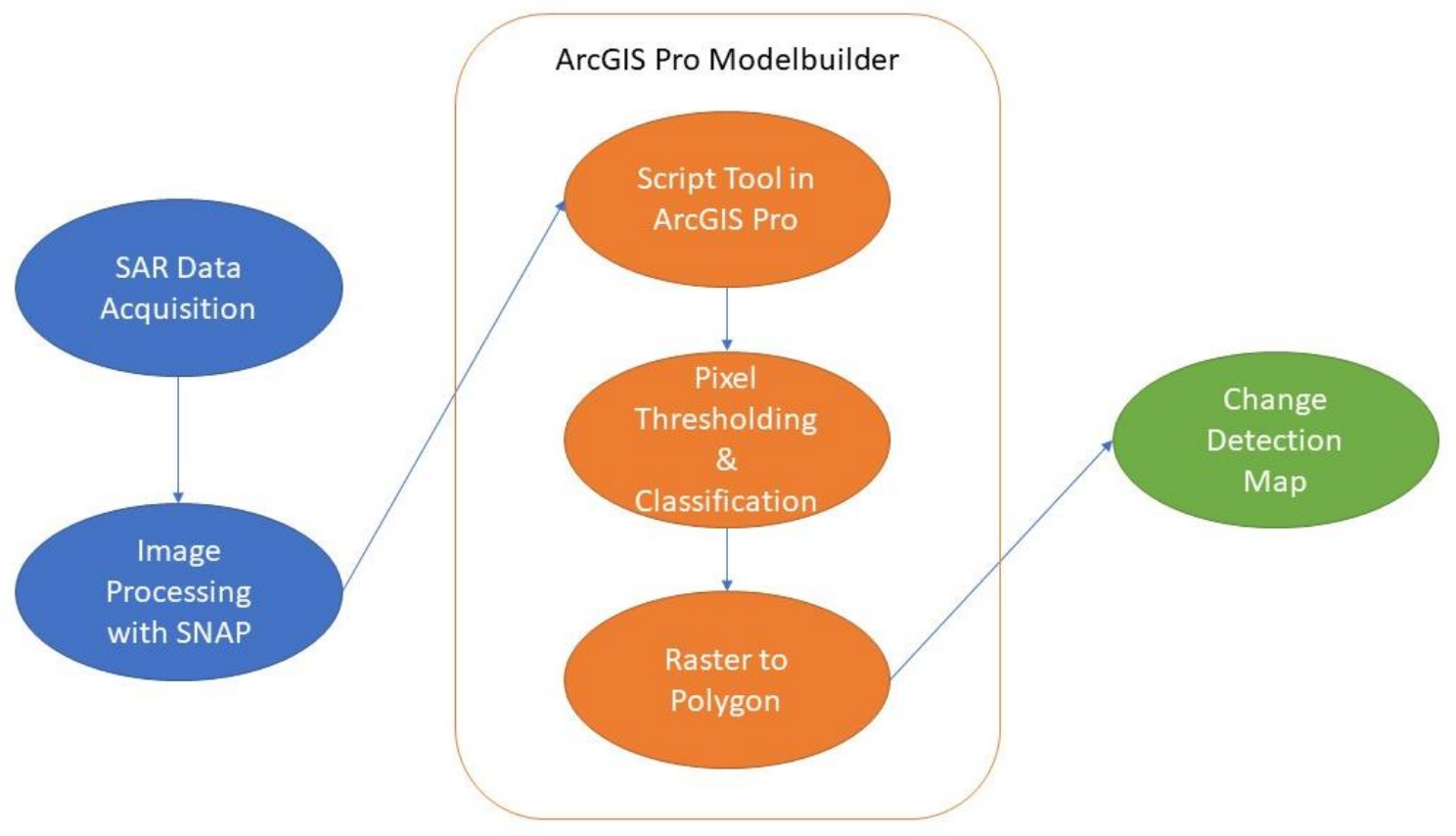

Figure 3-1: A diagram depicting the system design. 


\subsection{Project Plan}

The first conceptual ideas behind this project were focused more around the scientific analysis of land cover change in the study area over several years, rather than the development of a sound tool and methodology that would make that process doable for a range of users.

The original project plan aimed to automate classification analysis of Synthetic Aperture Radar (SAR) imagery every month from 2014 to the present, and then pass that information into a statistical raster analysis tool that could quantify the rates of deforestation and forest succession since the beginning of the Sentinel Mission in 2014. The plan was also to use Landsat imagery to calculate Normalized Difference Vegetation Index (NDVI) and gather optical imagery which was to be used to make separate classification values for spectral data, so that it could be passed to a raster analysis tool which would have the same functions as the one previously mentioned. In addition, the Time Slider function in ArcGIS Online was anticipated to animate the process of deforestation and create land cover maps on a three to five-year increment. The results from this were to be passed to ArcGIS StoryMaps to provide a complete story-telling application for the client.

The original project plan would have included significant amounts of scripting to automate processes of downloading data and running all the necessary preprocessing within ArcGIS Pro. It would have also required an analysis of the statistics and mathematics that would be incorporated into a script in order to automatically discern a classification schema based on optical data as an informant. Not only would these pieces of scope be beyond the level and requirements of the project, they would also take much longer to complete than the timeline for the project.

The project was accomplished by focusing more on the creation of a simplified analysis workflow and interface that would accurately assess changes to forest cover, so that the client could use it for whichever dates and increments of time that they chose. Additionally, optical imagery was collected using a drone. This field data was used as geographical informants to decide the thresholding values for change. With groundtruthing data available from 2017 to 2019, these were the dates and times that were used to create preliminary land cover change maps that were a prototype of the methodology.

These original components and timeline of the project is depicted in a Gantt Chart in Figure 3-2. 


\begin{tabular}{|c|c|c|c|c|c|}
\hline \multirow[b]{2}{*}{ Preparation } & \multirow{2}{*}{$\begin{array}{c}\text { ASSIGNED } \\
\text { TO }\end{array}$} & \multicolumn{2}{|c|}{ PROGRESS } & \multirow[t]{2}{*}{ START } & \multirow[t]{2}{*}{ END } \\
\hline & & & & & \\
\hline Gathering Sentinel Data & Abby & & $0 \%$ & $12 / 6 / 18$ & $1 / 5 / 19$ \\
\hline Gathering Mamoni Data & Claus \& Orlando & 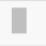 & $10 \%$ & $12 / 6 / 18$ & $1 / 6 / 19$ \\
\hline Research SAR \& Optical Analysis & Abby & $\square$ & $15 \%$ & $12 / 6 / 18$ & $1 / 7 / 19$ \\
\hline Research Geodatabase Design & Abby & 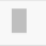 & $10 \%$ & $12 / 6 / 18$ & $1 / 8 / 19$ \\
\hline Choose GDB Template & Abby & & $0 \%$ & $12 / 6 / 18$ & $1 / 9 / 19$ \\
\hline \multicolumn{6}{|l|}{ Geodatabase Building } \\
\hline Identify Components for Input & Abby & F & $50 \%$ & $12 / 7 / 18$ & $12 / 11 / 18$ \\
\hline Identify Analyses to Take Place & Abby & & $50 \%$ & $12 / 9 / 18$ & $12 / 14 / 18$ \\
\hline Automate Analyses & Abby & & $0 \%$ & $12 / 14 / 18$ & $12 / 17 / 18$ \\
\hline Client Approval & Claus & & $50 \%$ & $12 / 14 / 18$ & $12 / 16 / 18$ \\
\hline Run Tests & Abby & & $0 \%$ & $12 / 14 / 18$ & $12 / 17 / 18$ \\
\hline \multicolumn{6}{|l|}{ Analysis } \\
\hline Drone Flights in Panama & Abby \& Dan & - & $10 \%$ & 2/24/19 & $3 / 2 / 19$ \\
\hline Incorporate Drone Imagery Into GDB & Abby & & $0 \%$ & $3 / 2 / 19$ & $3 / 23 / 19$ \\
\hline Use ERDAS Imagine for SAR Land Cover Analysis & Abby & & $0 \%$ & $1 / 14 / 19$ & $3 / 25 / 19$ \\
\hline Compare Drone Imagery to SAR & Abby & & $0 \%$ & $3 / 1 / 19$ & $6 / 12 / 19$ \\
\hline \multicolumn{6}{|l|}{ Deployment \& Documentation } \\
\hline Automate Geodatabase Functions of SAR + Optical & Abby & & $0 \%$ & $1 / 14 / 19$ & $4 / 10 / 19$ \\
\hline Go To Panama with May Term Class & Abby & & $0 \%$ & $5 / 1 / 19$ & $5 / 31 / 19$ \\
\hline Document Successes and Failures & Abby & ॥ & $5 \%$ & $5 / 11 / 19$ & $6 / 5 / 19$ \\
\hline Finish Paper & Abby & 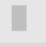 & $10 \%$ & $1 / 30 / 19$ & $7 / 19 / 19$ \\
\hline Finish Story Map & Abby & & $0 \%$ & $4 / 1 / 19$ & $6 / 30 / 19$ \\
\hline
\end{tabular}

Figure 3-2: Original timeline in a Gantt Chart

\subsection{Summary}

The purpose of this chapter was to discuss the reasons for the project and the needs that were presented by the client. The next point was to show how those needs were analyzed and thought out, and how the project design was created. A discussion on how the project design worked, and how it didn't work followed. Ultimately, the project was completed in a way that met the requirements and did so in an efficiently timed manner. The following sections will go into further detail about how the project was carried out and implemented. 


\section{Chapter 4 - Database Design}

This chapter will go into detail about the conceptual and logical models for the design and data of this project. Next it will discuss the data involved in the project including the sources, collection methods, data management and preprocessing necessary for the data to be used in the project.

\subsection{Conceptual Data Model}

A conceptual data model was needed to lay out all entities within the project and to show how they relate to each other. For a diagram of this model, see Figure 4-1.

In designing the model, there first needed to be a clear understanding of the general input entity and the output entity. For this project the main input was Synthetic Aperture Radar (SAR) imagery. The output was a map that depicted areas of change. To get there, two SAR images were needed to provide data from the beginning and ending dates of the time frame that was being analyzed for change. Next, those images were fed to an image analysis algorithm that would detect inconsistencies between the two dates (i.e., the two images). This algorithm created an image that showed the severity of inconsistencies between dates for each pixel. Then thresholds had to be applied to attempt to group neighboring pixels and create areas with clearer boundaries.

A second type of data for this model was drone imagery. Like the SAR imagery, two sets of drone imagery were input to represent two dates, which corresponded to the dates of the SAR imagery. A visual comparison of the two dates would determine areas of definite land cover change and the type of change that occurred. This result was used with the threshold image to give real-world meaning to the image analysis and determine the type of change that may be associated with the various values that came from the output of the image analysis.

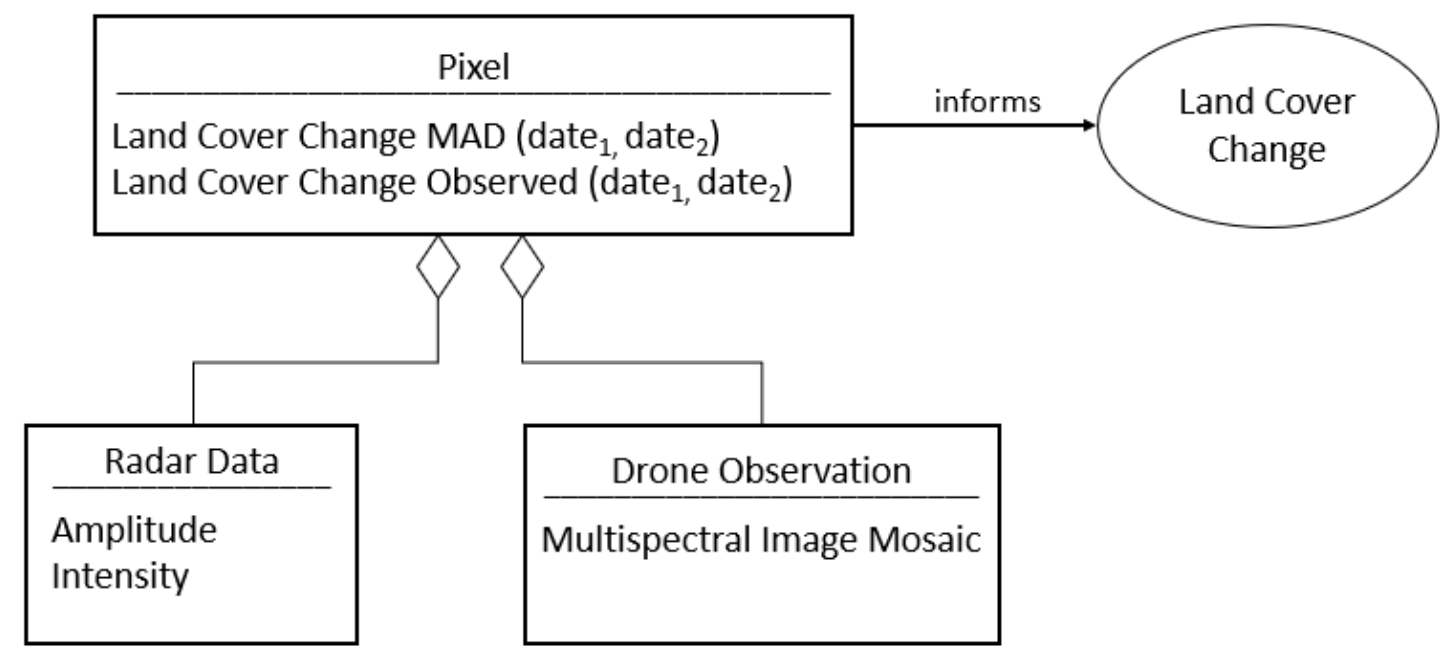

Figure 4-1: Diagram of Conceptual Model 


\subsection{Logical Data Model}

One, completely contained database was not created for this project although all pertinent entities did end in an ArcGIS Pro Project Geodatabase as Feature Classes, shown in Figure 4-2. Initially, image data was managed and stored through Windows File Explorer after it was downloaded from the European Space Agency's (ESA) Open Access Hub. The image data was downloaded in a zipped folder which was brought in directly to the SentiNel Applications (SNAP) interface. At each step of preprocessing in SNAP, a copy of the output image file was saved in the SNAP folder. The files were saved in BEAMDIMAP format, which is a simple input/output format and suitable within chains of data processing. Once preprocessing was completed, the final image was converted and exported through the SNAP interface into a tif file. The file was saved to a folder which also included an Esri ArcGIS Pro 2.4.0 Project, Mamoní Deforestation Tool.

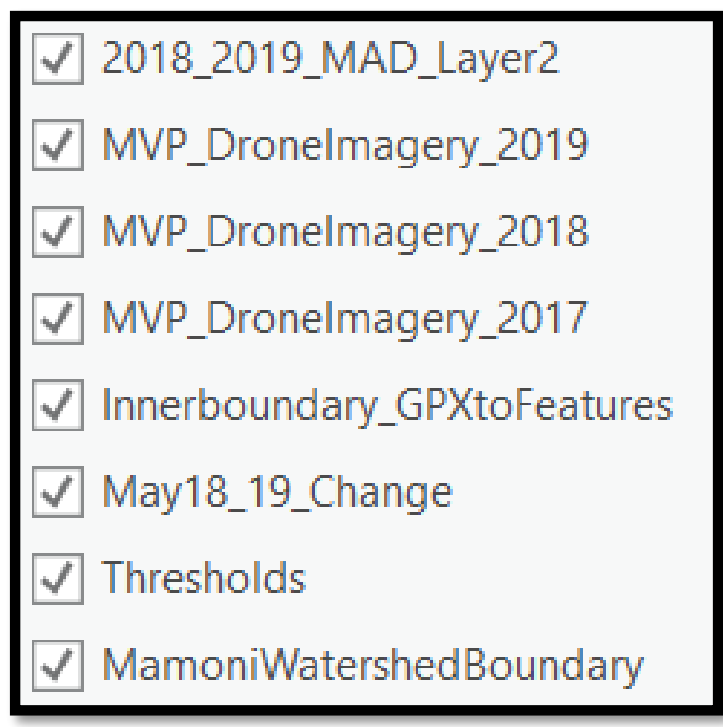

Figure 4-2: List of feature classes created and used for the project.

Drone imagery was acquired from a server controlled by the Center for Spatial Studies (CSS) and transferred to the local hard drive where the ArcGIS Pro Project and Feature Classes were also stored. Each drone image layer included the drone mosaic imagery, boundary and footprints of flights. The drone imagery was added to the Mamoní Deforestation Tool Project Geodatabase.

Additional feature classes included reference data such as the Mamoní watershed boundary which was also acquired from the CSS server and copied into the ArcGIS Pro Project. A separate boundary feature class was created to depict the rectangular study area that was specified by the client in geographic coordinates. This was the boundary that was used to extract pixels of the SAR data for Multivariate Alteration Detection (MAD) change analysis step. A feature class was created to contain changed-area polygons that would be digitized manually from a visual assessment of the differences 
between overlapping drone imagery. This feature class included one domain to specify the type of change, being gain or loss.

Next, threshold values were used to group pixels with similar pixel values. During the process, temporary layers were created and stored in the project geodatabase.

\subsection{Data Sources}

The primary data source for the project was the European Space Agency's Sentinel 1A Satellite within the Copernicus Earth Observation Program. The data is accessed through an Open Access Hub where the user can specify details about the data product such as orbit number, polarization, collection mode, product type and location which are all described in the following section, Section 4.4. Metadata for the Sentinel 1A satellite is available to the public on the program's website and product package zip file (sentinel.esa.int/web/sentinel/user-guides/sentinel-1-sar/data-formats/sar-formats).

Additionally, optical image data was used. This data was acquired with the DJI Phantom 4 Unmanned Aerial Vehicle (UAV). The UAV, also known as drone, flights were conducted by the project manager and the CSS at the University of Redlands. Metadata was recorded during flights to document the time, date, location, flight times and durations as well as any mishaps such as the UAV overheating, rapidly losing battery or straying from the planned flight path. This metadata is stored with the UAV mosaic imagery for ArcGIS Pro as well as in a digital document on the CSS server at the University of Redlands.

As stated in the previous section, a polygon boundary layer was copied from a CSS ArcGIS Pro Project on the CSS server. Geographic coordinates of the study area were collected directly from the client.

\subsection{Data Collection Methods}

The most important data in this project came from the ESA's Sentinel 1A Satellite in the Copernicus Program's Open Access Hub, shown in Figure 4-3. SAR sends a signal and records the returning signal's phase and intensity characteristics. This information is stored in a complex number. Phase refers to the starting point of a periodic wave relative to the origin of the wave. Intensity, also referred to as magnitude, is the strength of the returning radar signal. When acquiring Sentinel 1 data, there were several specifications that needed to be used to get the correct type of SAR data out of the options. 


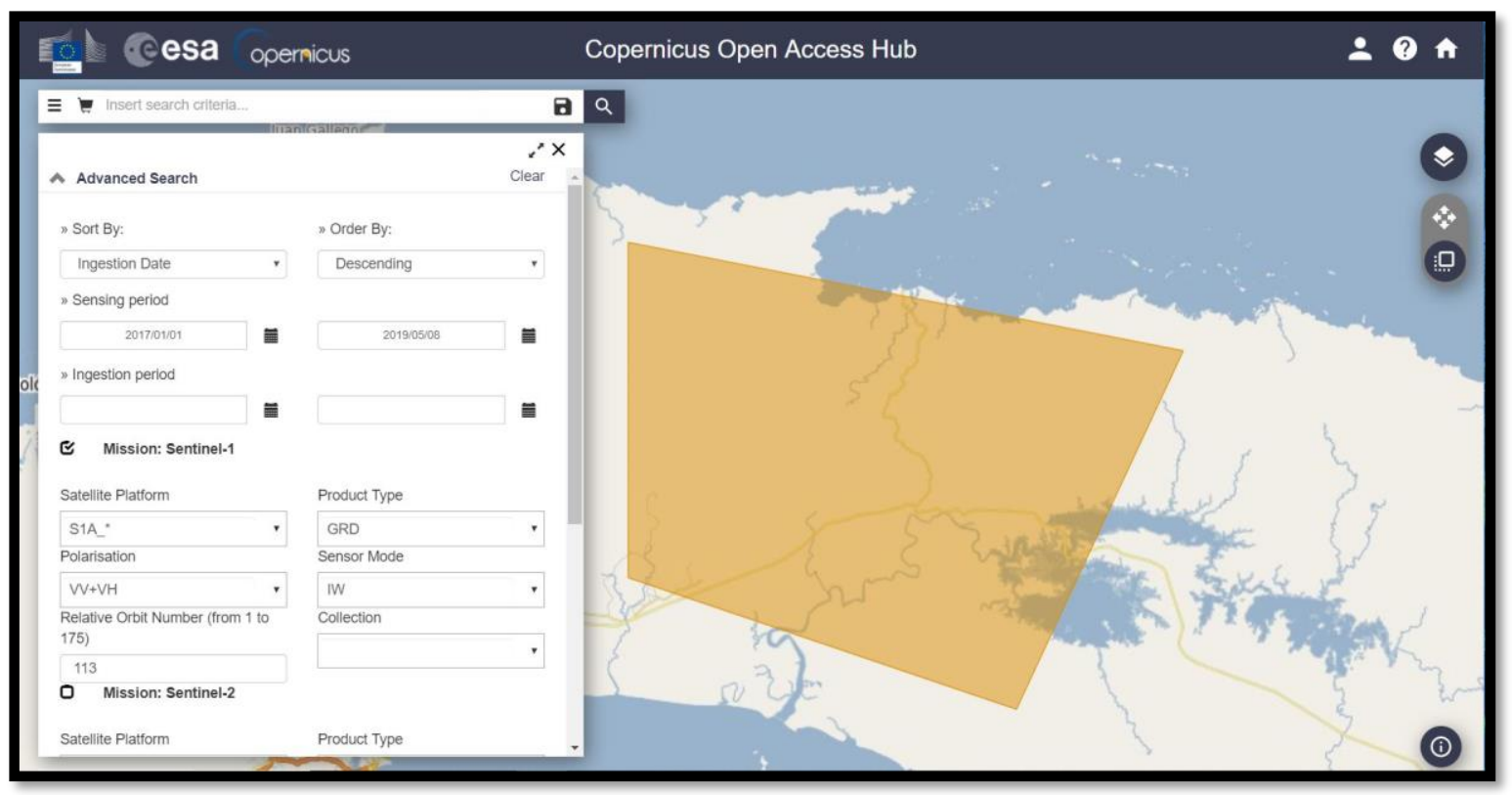

Figure 4-3: A Screenshot of the Copernicus Open Access Hub and filtering options.

The first and most significant specification was the product type. For the project, Ground Range Detected (GRD) products were used. This type of product consists of SAR data that has already been preprocessed through calculations of the complex number (inherent to a raw SAR image) to project the data to the ground using an Earth ellipsoid model (European Space Agency, 2015). This step is called multi-looking, and essentially turns the numbers contained in a raw SAR recording into a recognizable image, rather than noise. At this step, phase information is lost, simplifying the data and resulting in a product with a spatial resolution and pixel spacing that is approximately square (European Space Agency Sentinel Applications Platform, n.d.). This product type is originally a Single-Look Complex (SLC) product before the described preprocessing occurs. An SLC product contains phase information in addition to intensity information, which is stored in a complex number. GRD products were chosen in the data collection step because intensity information would be enough to use in the change detection analysis and would simplify the processing steps of the system, and simplicity was one of the client's desired system characteristics.

The next specifications in data collection were for acquisition mode and polarization. The ESA provides dual-polarity options, including $\mathrm{HH}, \mathrm{HV}, \mathrm{VV}$, and $\mathrm{VH}$ polarizations. The acquisition modes available are Strip Map (SM), Interferometric Wide Swath (IW), Extra-Wide Swath (EW), and Wave (WV) Mode. The mode chosen was IW with a polarity of $\mathrm{VV}+\mathrm{VH}$ because it was recommended by the ESA as the primary conflictfree mode over land. Additionally, IW was the only acquisition mode available for the study area.

Finally, the orbit number was specified. This ensured that both products used for change detection had data that was recorded when the satellite was in the same position. This decreased the need for additional preprocessing to align the two products accurately when stacking the images for analysis. The relative orbit number specified was 121 , 
because it had the most optimal fit for the study area compared to the other available orbit number, which was 113, as seen in Figure 4-4.
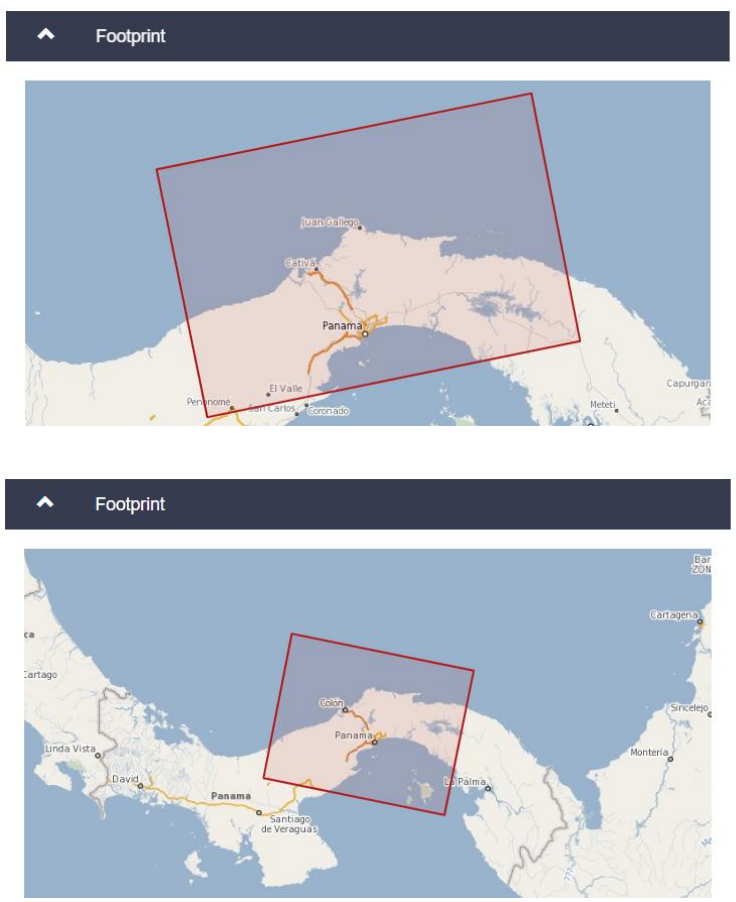

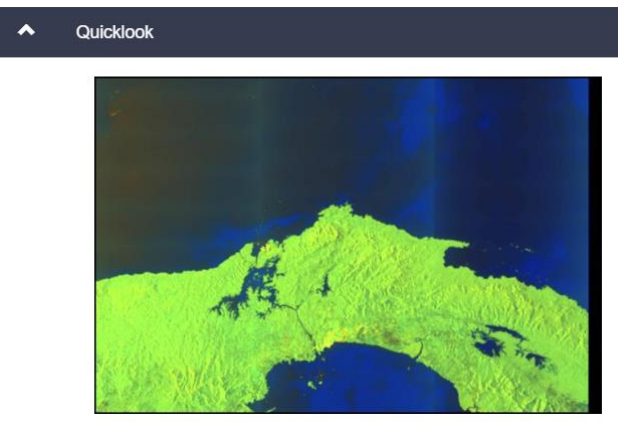

Auicklook

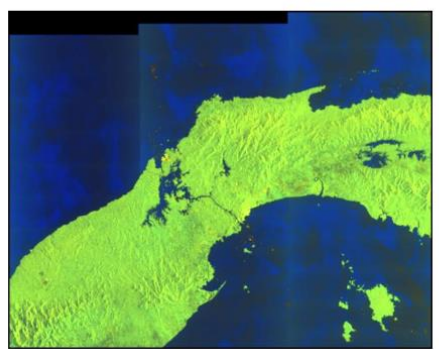

Figure 4-4: Snapshots from the Copernicus Open Access Hub, showing the footprint and Quicklook of SAR products with the orbit number 121 (top) and orbit number 113 (bottom).

\subsection{Summary}

This chapter aimed to provide an overview of the conceptual data model and the entities that were important to this project's functionality and purpose. Next, more detail was provided through describing the logical data model and the characteristics of the database components. Following this, details regarding data sources, collection methods, and cleaning were given in depth to provide a significant understanding of the data that went into the project. 



\section{Chapter 5 - Implementation}

This chapter will discuss the various incremental steps of the project and how they led to project deliverables and completion. Beginning with the first step of data identification, the chapter will then describe the initial steps of processing that would aim to warrant accurate results. Next, the development and implementation of the change analysis will be explained, as well as the way in which drone data was used to validate the results.

\subsection{Identification of Data Sets}

Because Unmanned Aerial Vehicle (UAV) imagery, also known as drone imagery, was the method of validating analysis, it was also a limiting factor to the Synthetic Aperture Radar (SAR) datasets that could be used for the project. This meant that the SAR imagery needed to coincide in the times that drone imagery was gathered. In 2017, 2018 and 2019, multispectral drone imagery was gathered in May. SAR imagery needed to be collected from the same time frames. As shown in Figure 5-1, more drone imagery was collected in 2018 and 2019, so collecting SAR imagery from May 2018 and May 2019 was the best option for comparing two dates and would have a better opportunity for validation. 

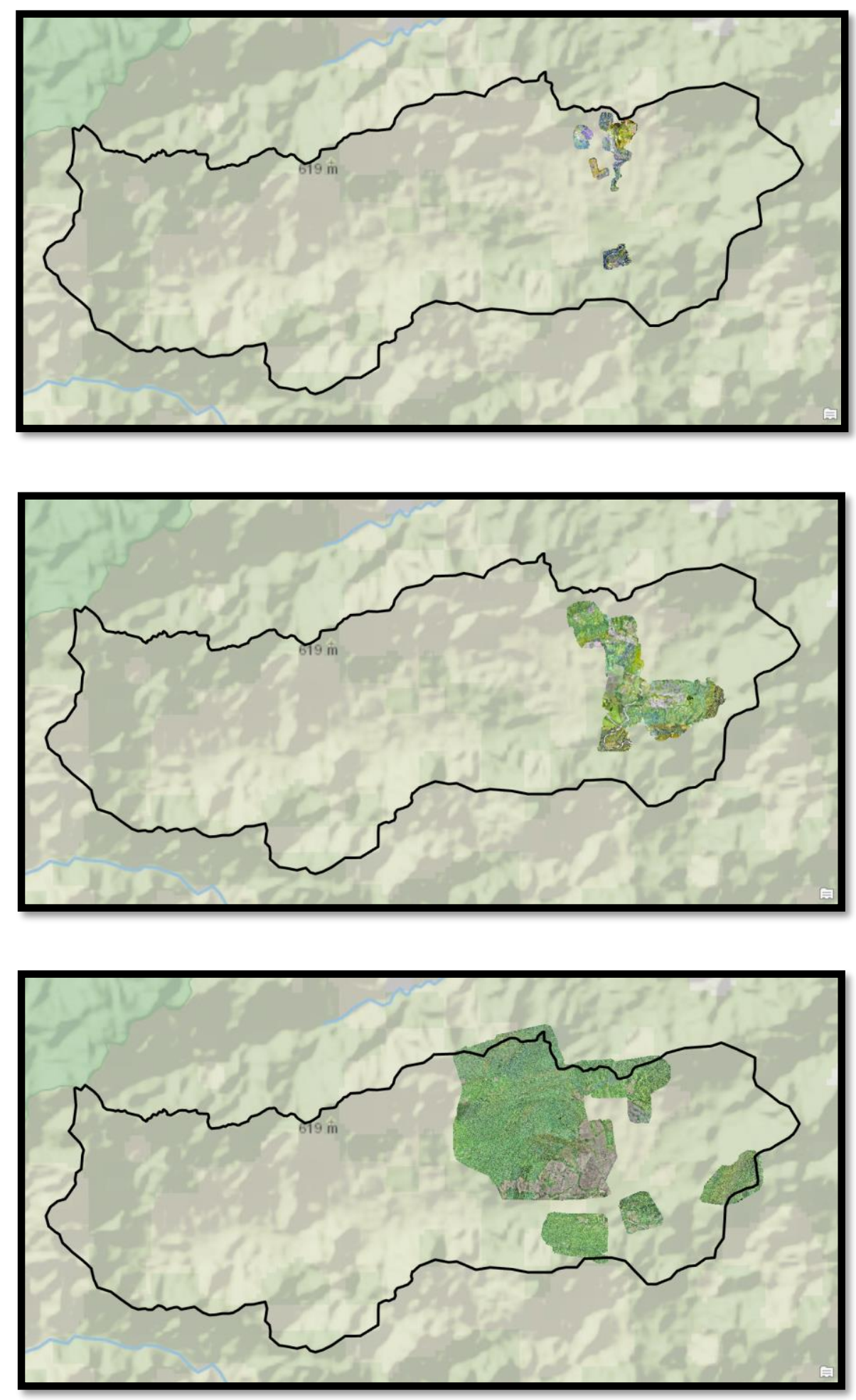

Figure 5-1: Drone imagery acquired from 2017 (top), 2018 (middle), and 2019 (bottom). 
Another reason for choosing this is that between these two years, there was also the greatest amount of geographic overlap between flights, as seen in Figure 5-2.

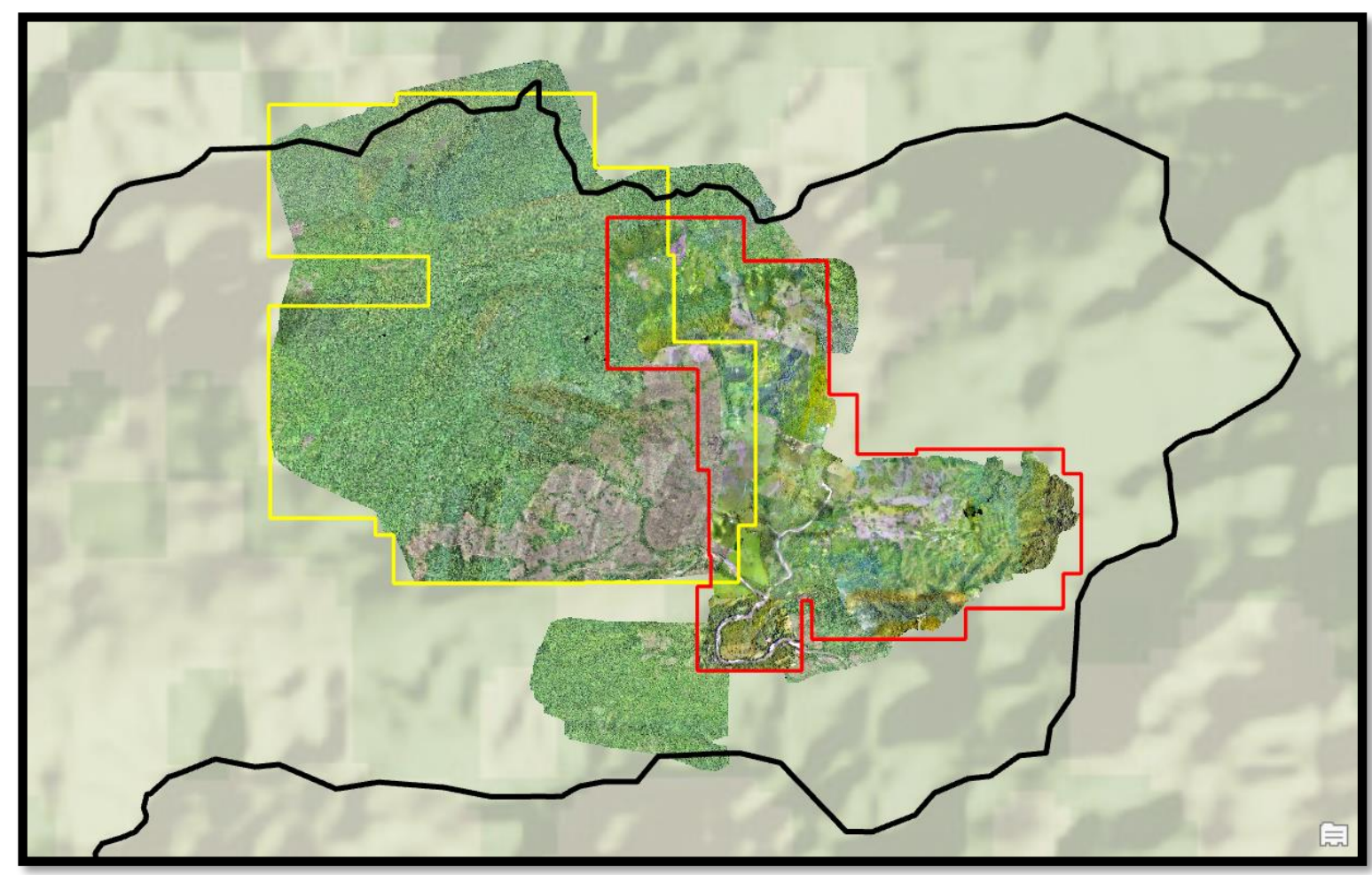

Figure 5-2: The approximate overlap depicted by orthomosaic boundaries of drone imagery from 2018 (red) and 2019 (yellow).

\subsection{Preprocessing SAR Data}

Several steps of SAR preprocessing were necessary. However, some typical processes were bypassed because the two SAR products needed be clean and uniform enough to be accurately compared to identify locations of discrepancies.

The first step of preprocessing was terrain correction. This is like an orthorectification process which transforms and corrects distortions in an image that are caused by topographic variations. This is necessary because the tilt of the satellite sensor, as well as topographical variations of a scene being recorded, will lead to distortions in pixel size, creating a non-uniform scale in a SAR image, represented in Figure 5-3. Relief displacement is another term used for this issue in image processing. In the figure below, the radar signal is sent out from an entity at $\mathbf{S}$. It travels a distance of $\mathbf{R}$ to reach points $\mathbf{B}$. $\mathbf{B}$ represents a point with elevation $\mathbf{h}$ above the ellipsoid model of the earth. It is imaged at position B' in a SAR image, though its real position in B'. $\Delta \mathbf{r}$ represents the offset between B' and B'; the effect of topographic distortions. Terrain correction also allows geometric overlays of image data from different sensors and geometries (European Space Agency Sentinel Applications Platform, n.d.). 


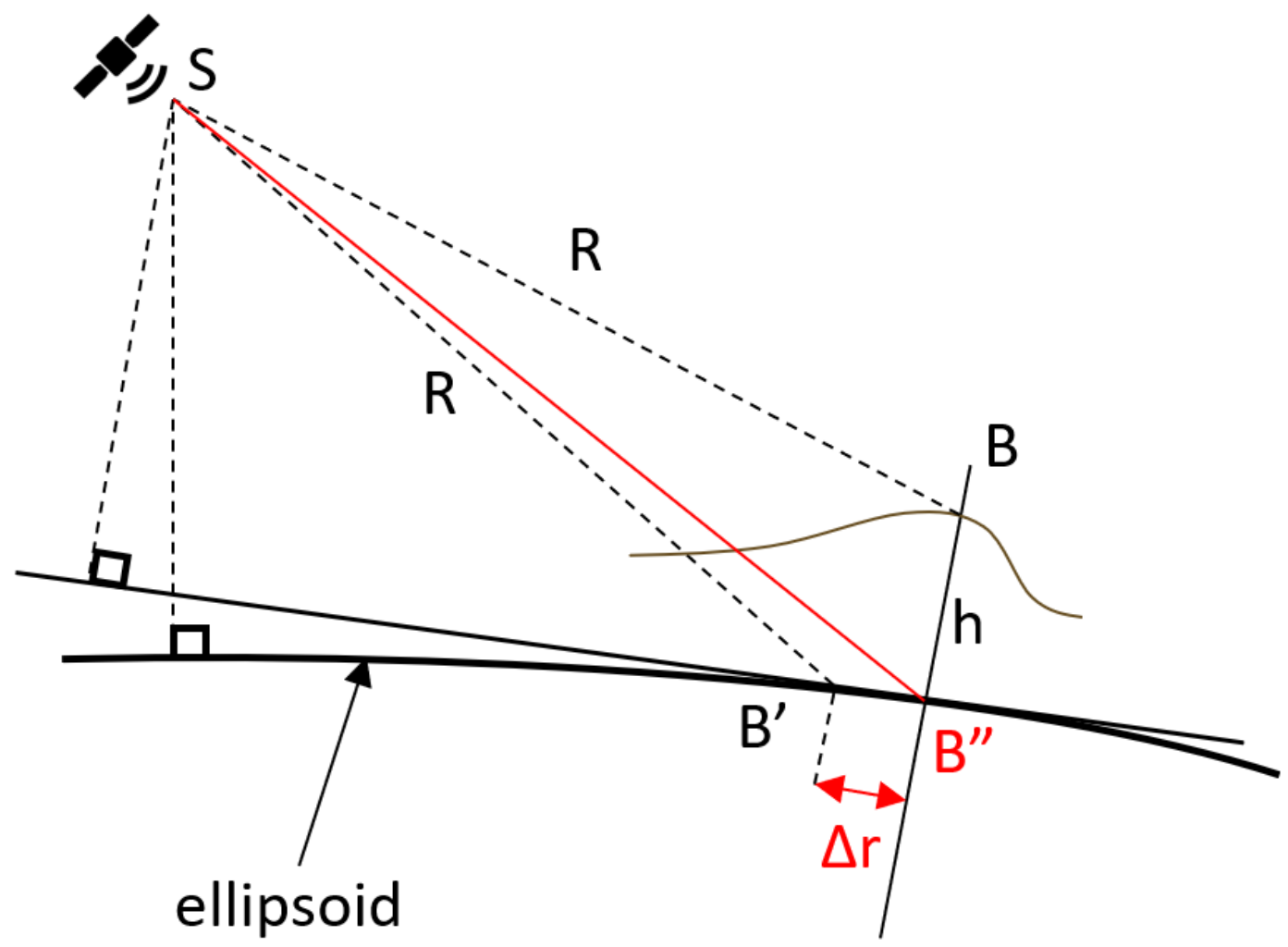

Figure 5-3: Diagram of SAR geometry that leads to distortion (adapted from European Space Agency Sentinel Applications Platform, n.d.)

Terrain correction aims to compensate for the distortions inherent to SAR geometry. It also re-projects the image data into a geographic coordinate system, georeferencing the image so that the image geography appears as close as possible to a uniform scale. This process is similar to optical image orthorectification, removing relief distortion, illustrated in Figure 5-4. 

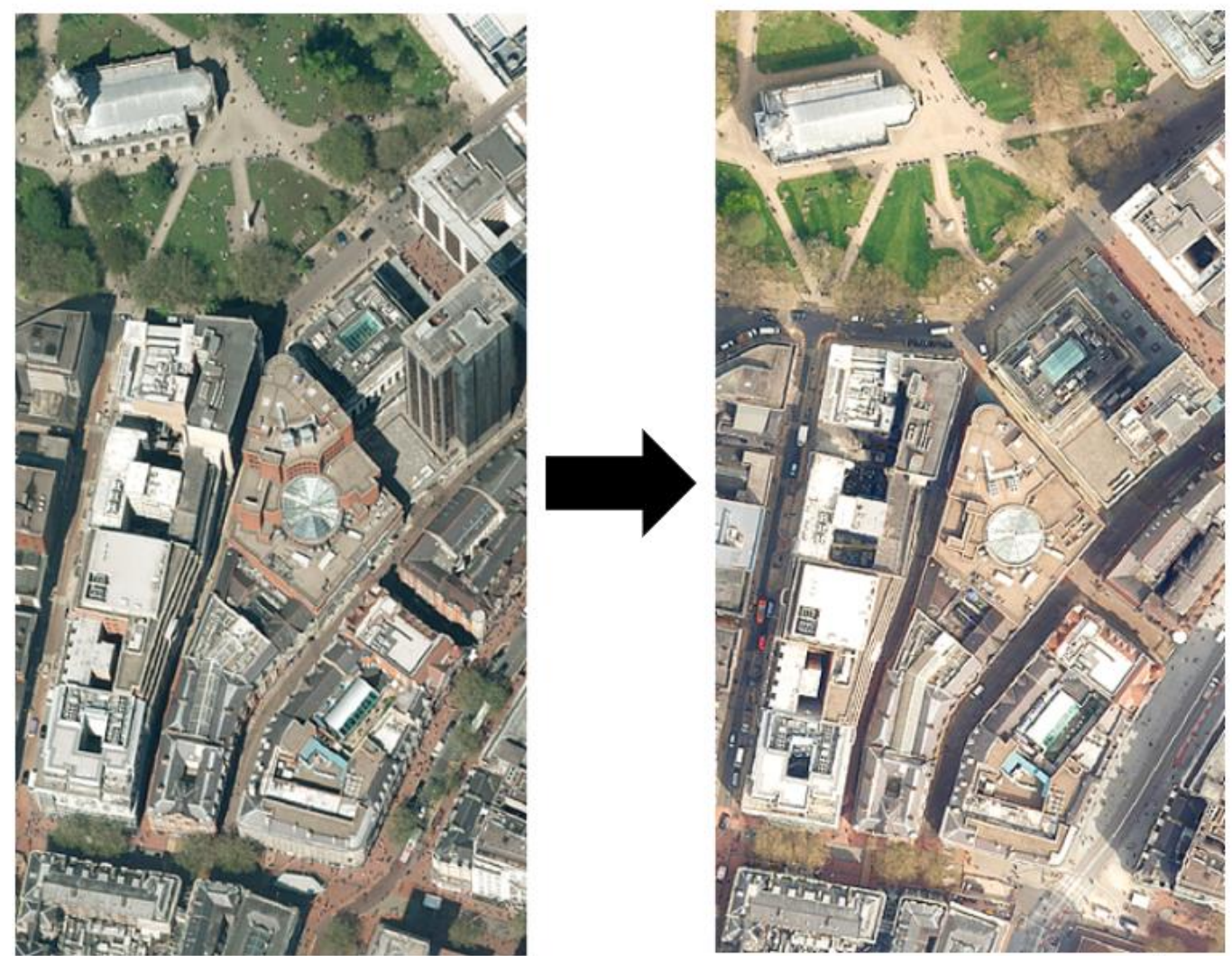

Figure 5-4: Before (left) and after (right) distortion correction in a city scene (Engineer's Journal, 2018).

The SAR product used in the project before and after terrain correction is shown in Figure 5-5.

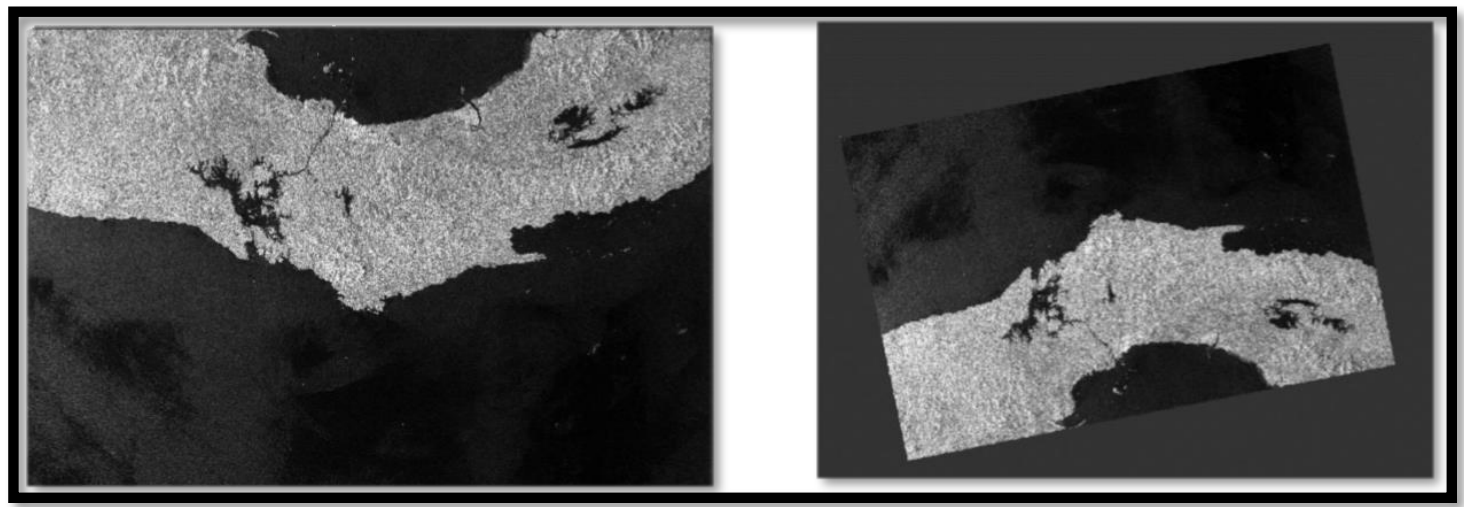

Figure 5-5: Before (left) and after (right) terrain correction. 
After terrain correction, the two SAR image products were stacked. This was necessary for the Multivariate Alteration Detection (MAD) step that is implemented in this project as it takes both dated image products as input at once.

Next, the SAR data was clipped to a region that would be used for analysis. Global Positioning System (GPS) Exchange Format (GPX) points were given by the client. These points were used to mark the study area. The GPS point values were recorded in latitude and longitude, and those values were used in the European Space Agency's (ESA) SentiNel Applications (SNAP) toolbox while using the subset tool. This significantly cut down the size of the product (see Figure 5-6) which made all subsequent processing finish at faster speeds and made the change analysis more accurate.

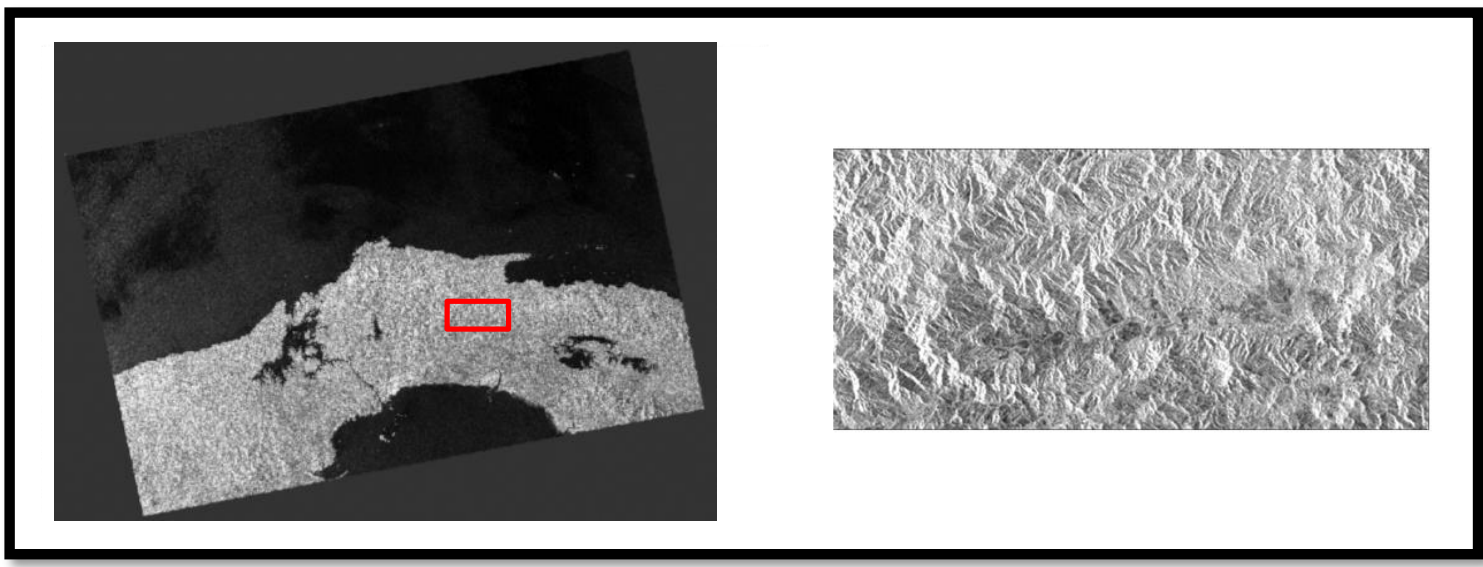

\section{Figure 5-6: Before and after using the subset tool to focus on the Mamoní Valley study area.}

The next step of preprocessing was speckle filtering. This was necessary because all SAR images have a noisy texture. This is caused by interference of return radar waves that are scattered within each resolution cell, and the interference causes degradation of the quality of the image. Of the various speckle filtering procedures, Multi-temporal Lee Sigma was implemented. Multi-temporal speckle filtering was used because this would ensure consistency with each layer of the multi-temporal image stack. The Lee Sigma filter uses the statistical distribution of the digital number values within a window of an image to determine what the pixel of interest should be. It is based on the probability of a normal, Gaussian distribution, and assumes that $95.5 \%$ of random samples within a window are within a standard deviation of 2 (2 sigma). The filter then replaces a pixel of interest with the average of all the digital number values within a window of an image. This is done throughout the image, to smooth the appearance and pixel values. After speckle filtering, the SAR image could be interpreted more clearly as seen in Figure 5-7. 


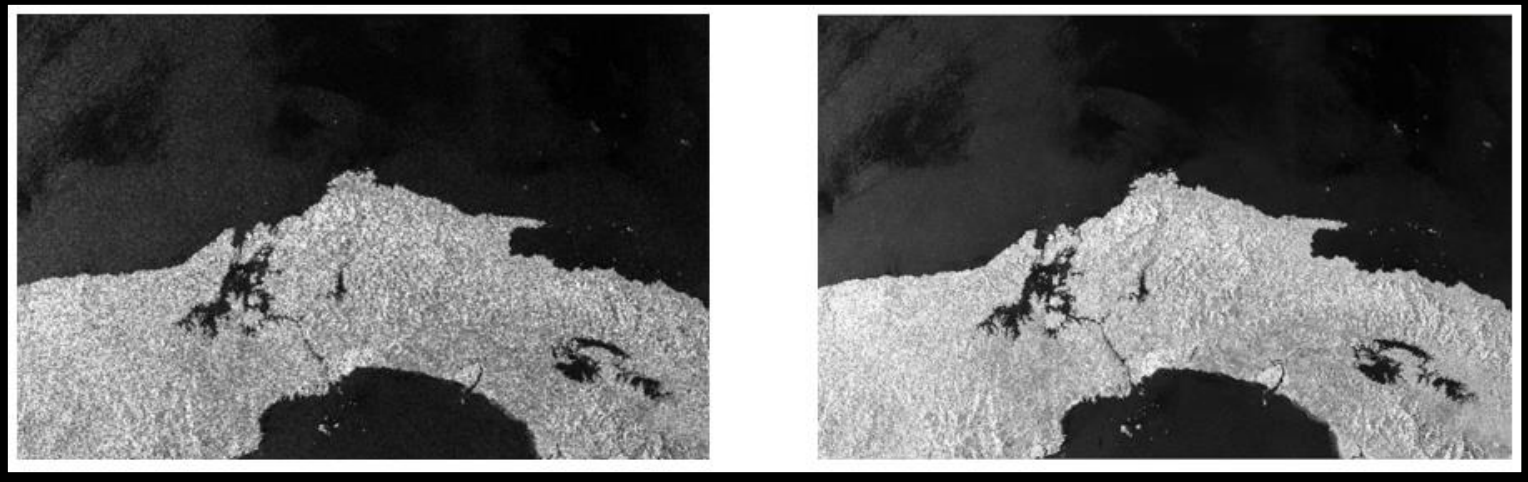

Figure 5-7: Before (left) and after (right) speckle filtering. The full extent of the product is shown here to best display the changes made by speckle filtering.

Finally, the stacked and preprocessed product was exported as a GeoTiff file format into the folder where the ArcGIS Pro Project was also saved, ready for analysis.

\subsection{SAR Surface Change Detection Analysis}

MAD was used to find changes from one year to the next. To understand this method of analysis, refer to Section 2.3.1. This analysis was implemented in the Python Wing Integrated Development Environment (IDE) 101 6.1. This script was written by Professor $\mathrm{Ma}$, and can be seen in Appendix A. Once the script was executed, a resulting image in .tif format was generated and saved in the same file location of the original image. The SAR image before and after analysis can be seen in Figure 5-8. 

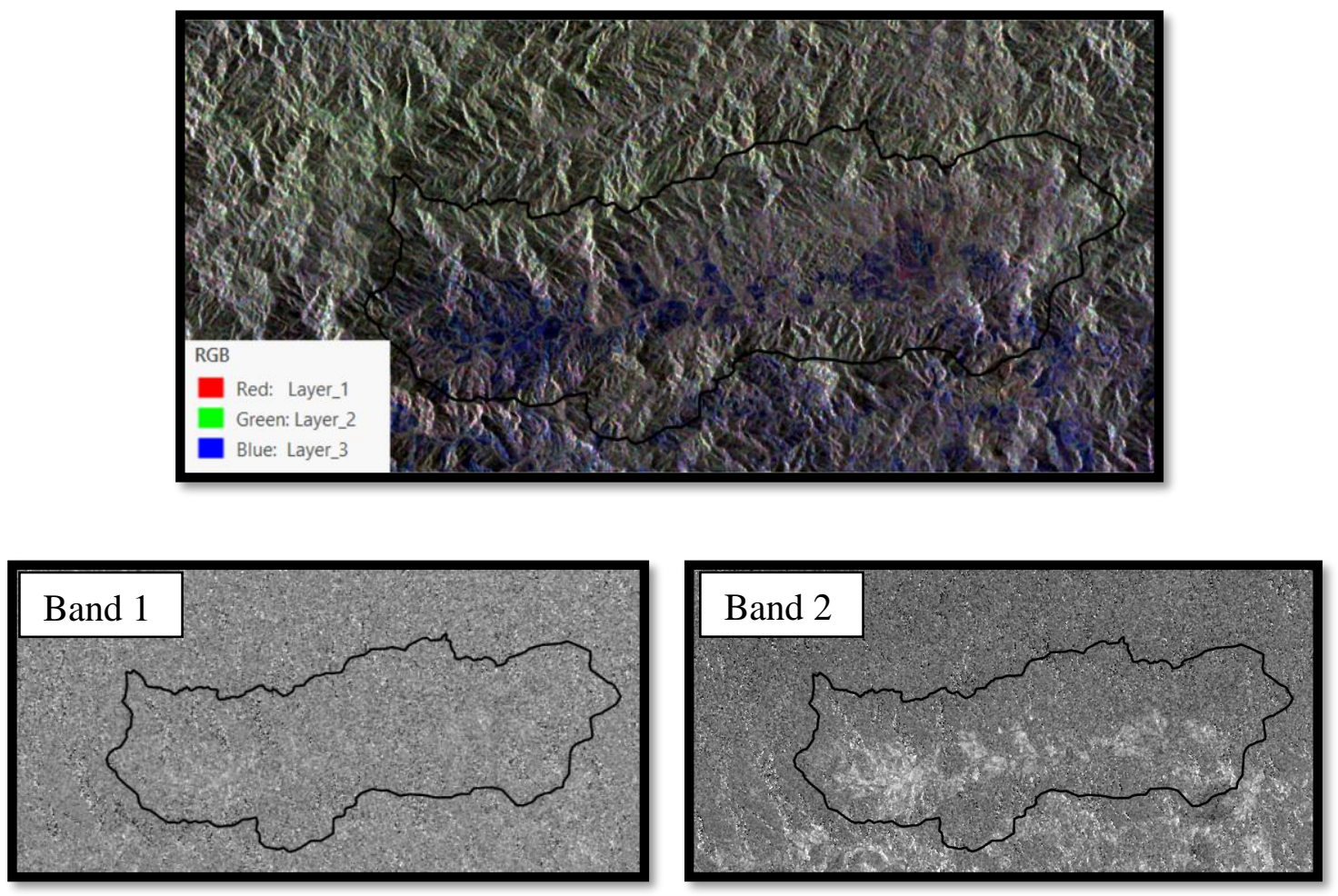

Figure 5-8: The original Ground Range Detected (GRD) images containing VV and VH polarized layers, preprocessed and stacked (top). First and second layers after MAD analysis (bottom).

Inherent to MAD, the listed pairs of canonically correlated pairs show the difference of the ordered outputs. In this case, there were two pairs for the two original bands of each input image. This meant that the first band of the output image was the most correlated and the second band of the output was the least correlated. Therefore, the last band was used to find areas that point to land cover change because this would presumably show the changed areas. For more information on this analysis, reference Section 2.3.1. As seen in Figure 5-8, the second band had more variation in pixel values, and showed more distinct patterns in the image, which may indicate different ways in which land cover had changed between 2018 and 2019. The pixels containing the highest absolute values would show the areas where there was the greatest difference between the two dated images.

\subsection{Multispectral UAV Imagery}

UAV imagery from 2018 and 2019 was used to find locations where there was a visible change in land cover between the two years. With the imagery that was available, there were several clear areas of change which were used to check against the MAD analysis. These areas were digitized in ArcGIS Pro 2.3. Three instances showed where vegetation was lost from 2018 to 2019. One instance showed where vegetation was gained. See these areas in the following Figures 5-9 to 5-11. In Figure 5-10, drone imagery cut off an 
area where local knowledge and typical land use practices led to the inference that the entire burned area shown in 2019 was a full stand of trees as shown in 2018.
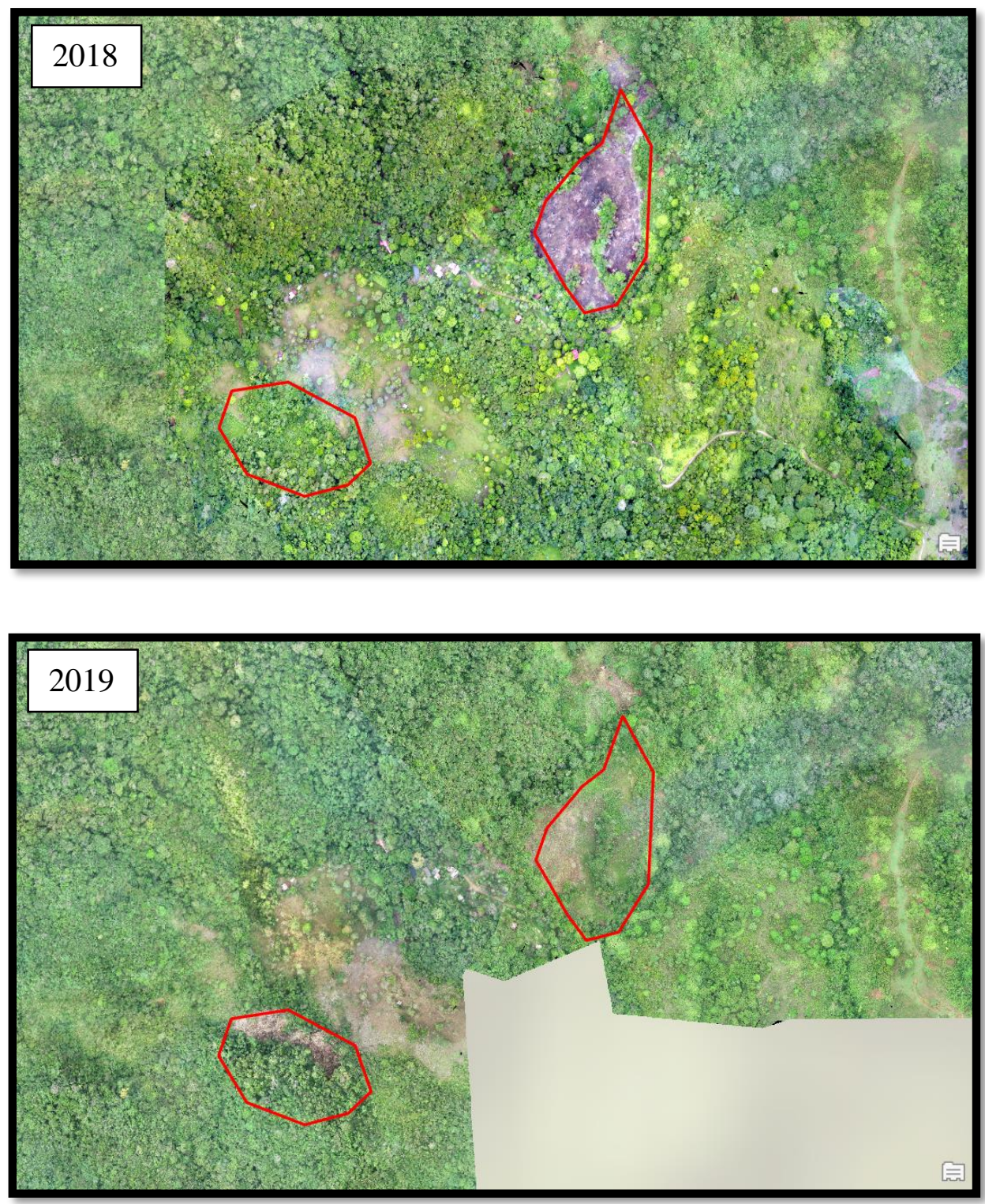

Figure 5-9: Two digitized areas, one (left) experienced vegetation loss from 2018 to 2019. The other (right) experienced vegetation growth from 2018 to 2019. 

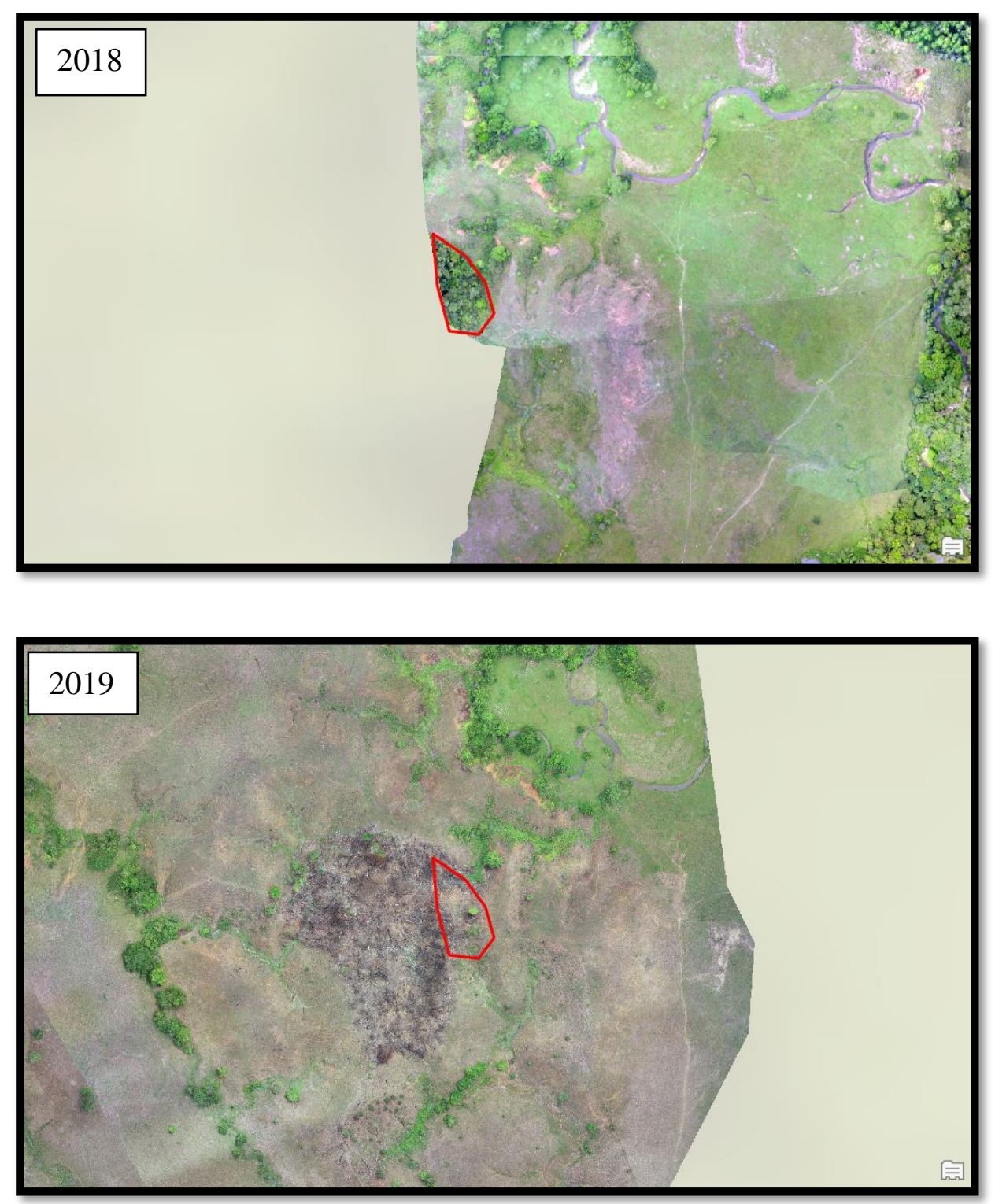

Figure 5-10: An instance of forest loss. 2018 UAV imagery cuts off in this area. 

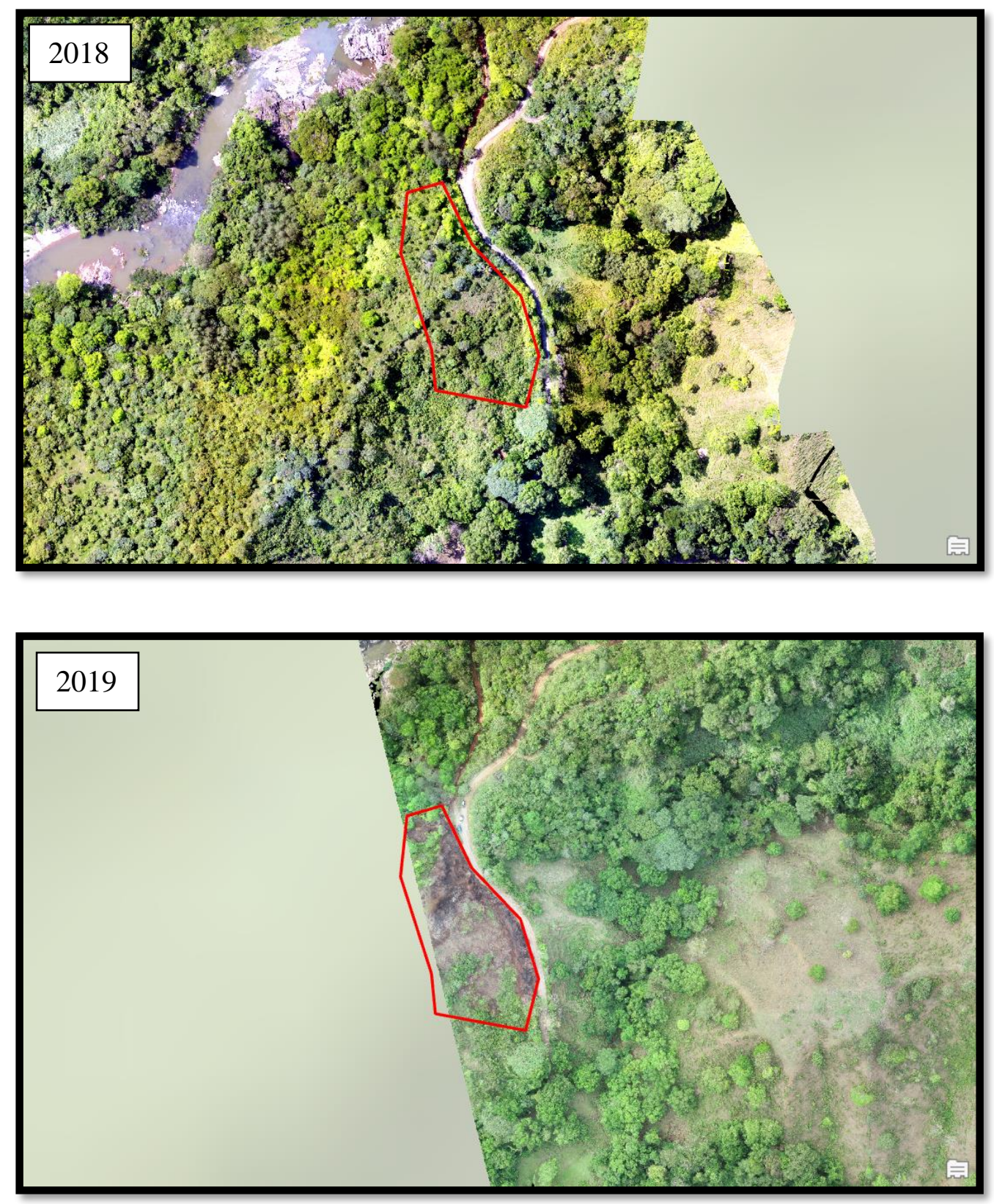

Figure 5-11: Another area of vegetation loss.

These polygons were overlaid onto the MAD result to determine whether patterns existed between changed areas shown by UAV and the changed areas shown by SAR analysis, as seen in Figure 5-12. 


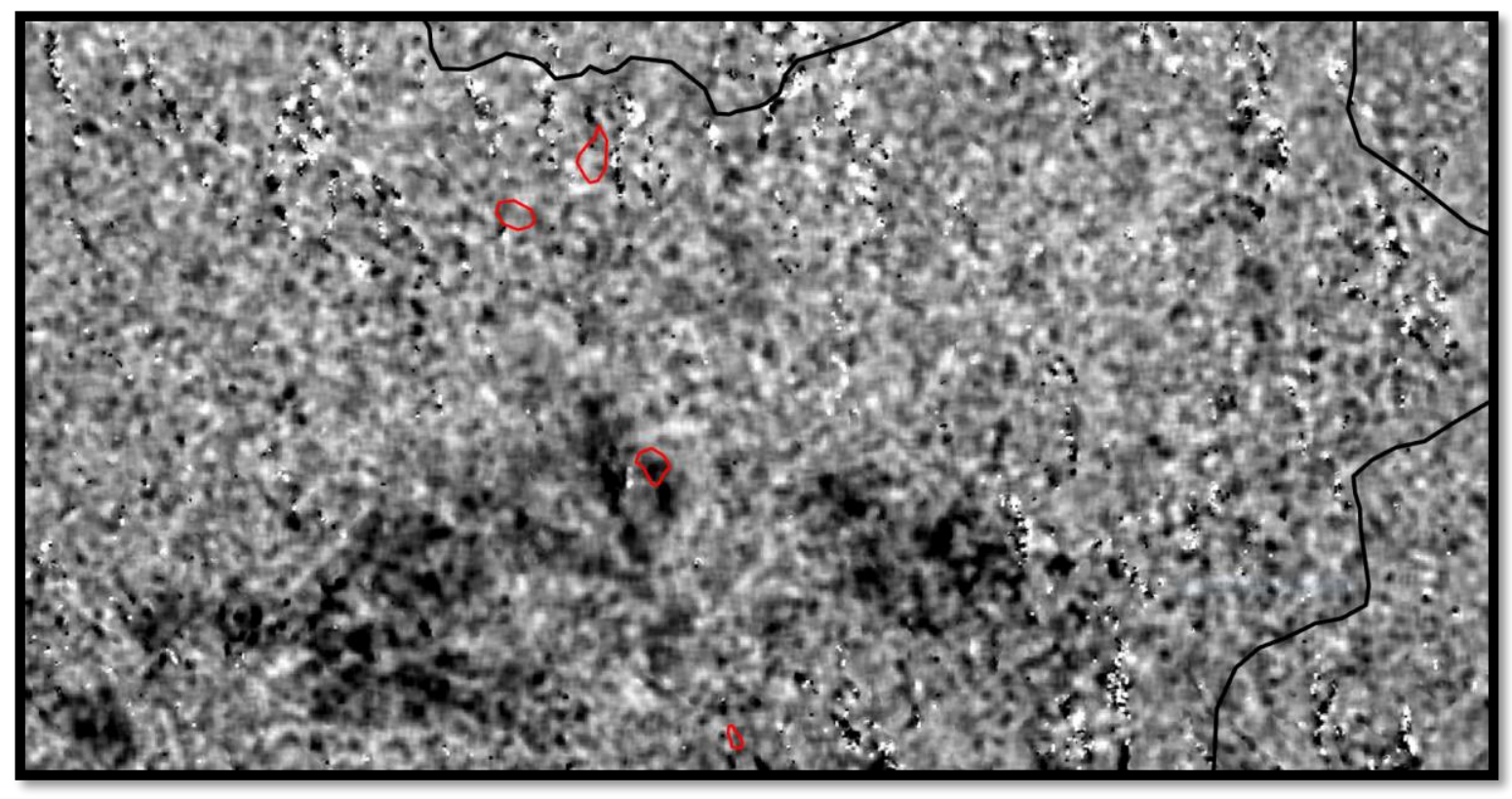

\section{Figure 5-12: UAV land cover change polygons distributed across MAD result in Mamoní Valley.}

Finally, threshold values were applied to group pixels with similar values to try to find discrete areas of land with similar characteristics. A high and low threshold value was applied to the raster, so that all pixels with a value less than or equal to -0.08 were grouped, and all pixels with a value greater than or equal to 0.4 were grouped. These thresholds also made any pixels that fell between the threshold values have a pixel value of 0 . This is because results close to 0 in the MAD analysis indicate no change. Drone imagery was used to choose these threshold values such that high values would be showing one type of forest change (such as degradation) and low values would be showing another type of forest change (such as growth). This allowed a visualization of the MAD results with pixels containing the highest absolute values and pixels containing the lowest absolute values. A diagram of this full workflow is shown in Figure 5-13. 


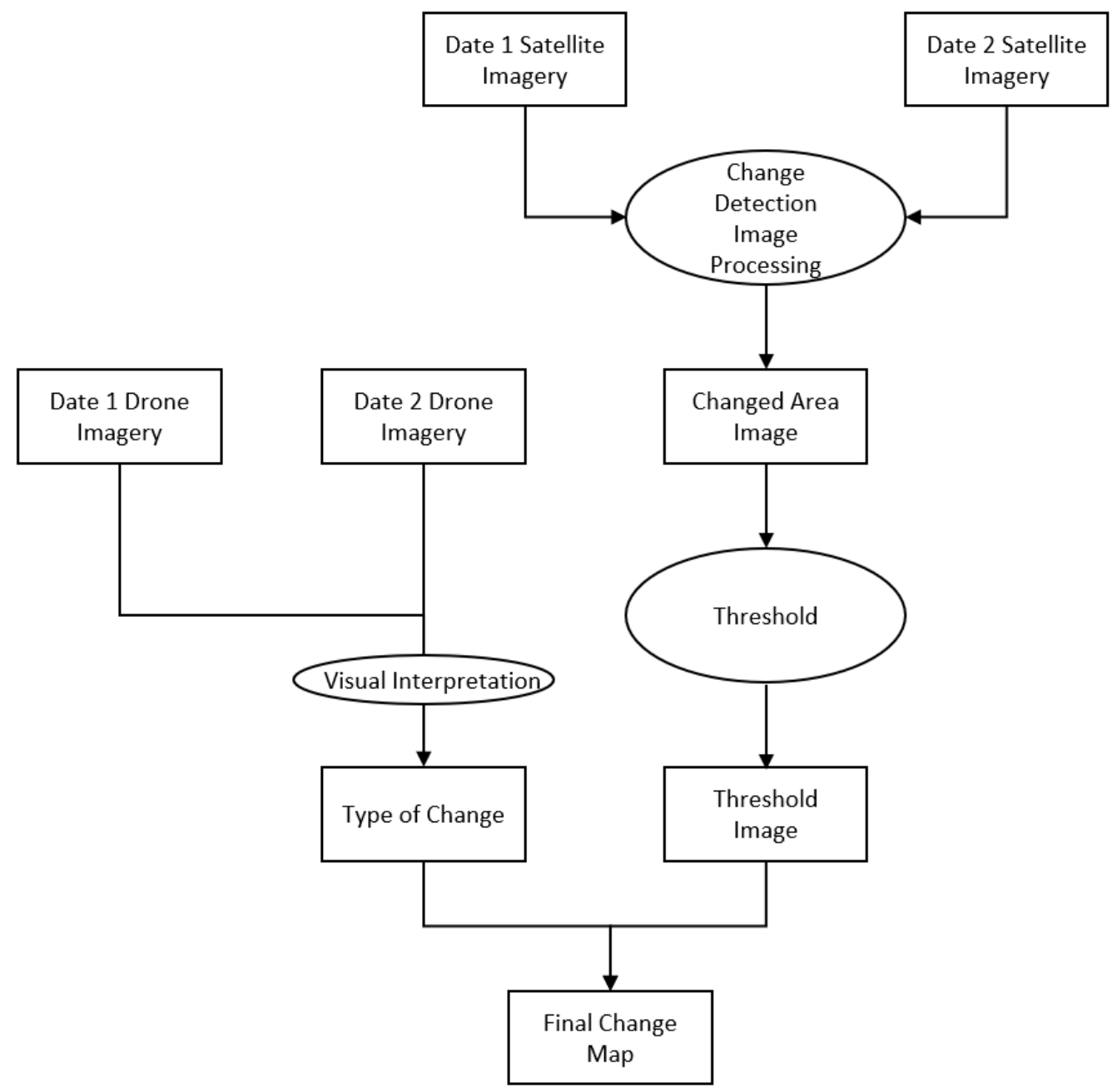

Figure 5-13: A diagram of the workflow used during project implementation.

\subsection{Automating Preprocessing and MAD Analysis}

In the SNAP toolbox, the Graph Builder tool was used to create a workflow that would automate all preprocessing steps. As one deliverable, the graph was saved in a computer file on an external hard drive which was designated for the project. It was saved as an .xml document which can be loaded directly into SNAP for future use. To view this document, see Appendix B. Each step of the workflow, shown in Figure 5-14, takes the input that is temporarily created from the previous step. Specifications for each step are also saved in the workflow, such as the coordinates of latitude and longitude for the subsetting step. 


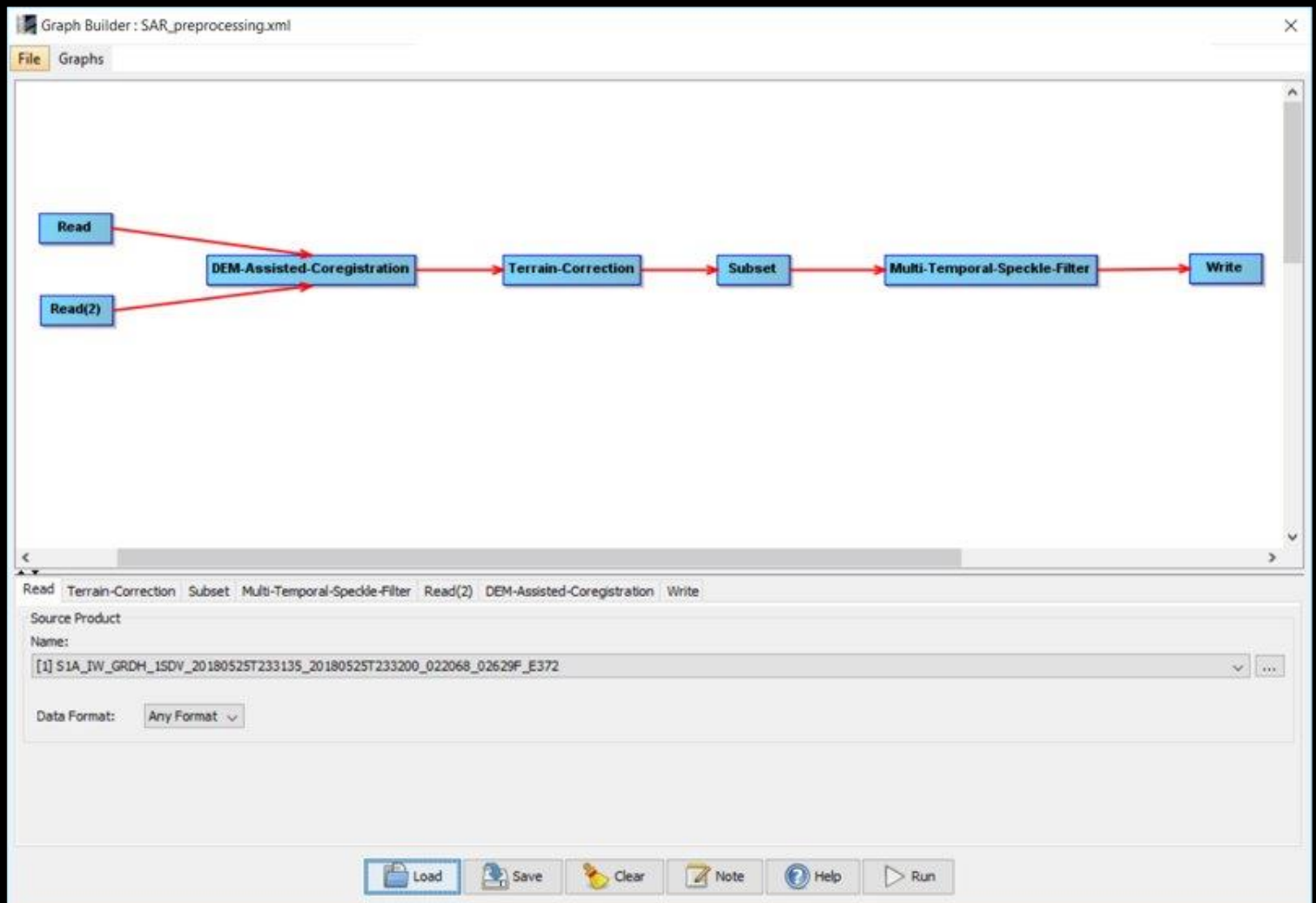

\section{Figure 5-14: Automated workflow for preprocessing.}

The preprocessed image was written out as a tif file into the designated folder on the hard drive. Next, the tif image could be used as input for the image processing workflow implemented in ArcGIS Pro as a Python Script Tool in Model Builder. This model prompts the user for the preprocessed SAR image and to specify the folder in which the output would be stored, shown in Figure 5-15. 
$\oplus \quad$ Multivariate Alteration Detection (Optional MAF Postprocessing)

Parameters Environments

Input SAR Stack (GeoTiff)

Output Folder

Figure 5-15: MAD script tool interface in ArcGIS Pro.

As a second deliverable, the script tool was built to automatically write the result into the ArcGIS Project Geodatabase that was created for the project. It also automatically displays the MAD result in the map viewer, allowing the user to implement future drone imagery and field observations as well as to inform thresholds.

\subsection{Summary}

This chapter discussed the processes that occurred to reach project requirements and develop project deliverables. First, analyzing what data could be used was necessary to achieve optimal results, which meant that the available UAV data needed to be compared to SAR data. Next, the steps and reasoning behind preprocessing SAR data was described as this would be a part of the final automated deliverable for the client. After preprocessing, the implementation of the change detection, MAD, was discussed as well as the way in which the result was analyzed through thresholding and multispectral UAV imagery. Finally, the creation and implementation of automated workflows in the SNAP Toolbox and ArcGIS Pro was described. 



\section{Chapter 6 - Results and Analysis}

This project tested a method of using Multivariate Alteration Detection (MAD) to detect land cover changes between Synthetic Radar (SAR) satellite images from two dates and Unmanned Aerial Vehicle (UAV) imagery, also known as drone imagery, to validate and identify the types of changes. This chapter will explain the results and implications of this project. Limitations of this project will also be addressed, accompanied by discussion of possible reasonings for the challenges that were present.

\subsection{MAD Results with UAV Control Areas}

Raw MAD results showed a change from the original input image, as seen in Figure 6-1. The pixel values varied from -11.63 to 10.86 , with some clustering of high pixel values.

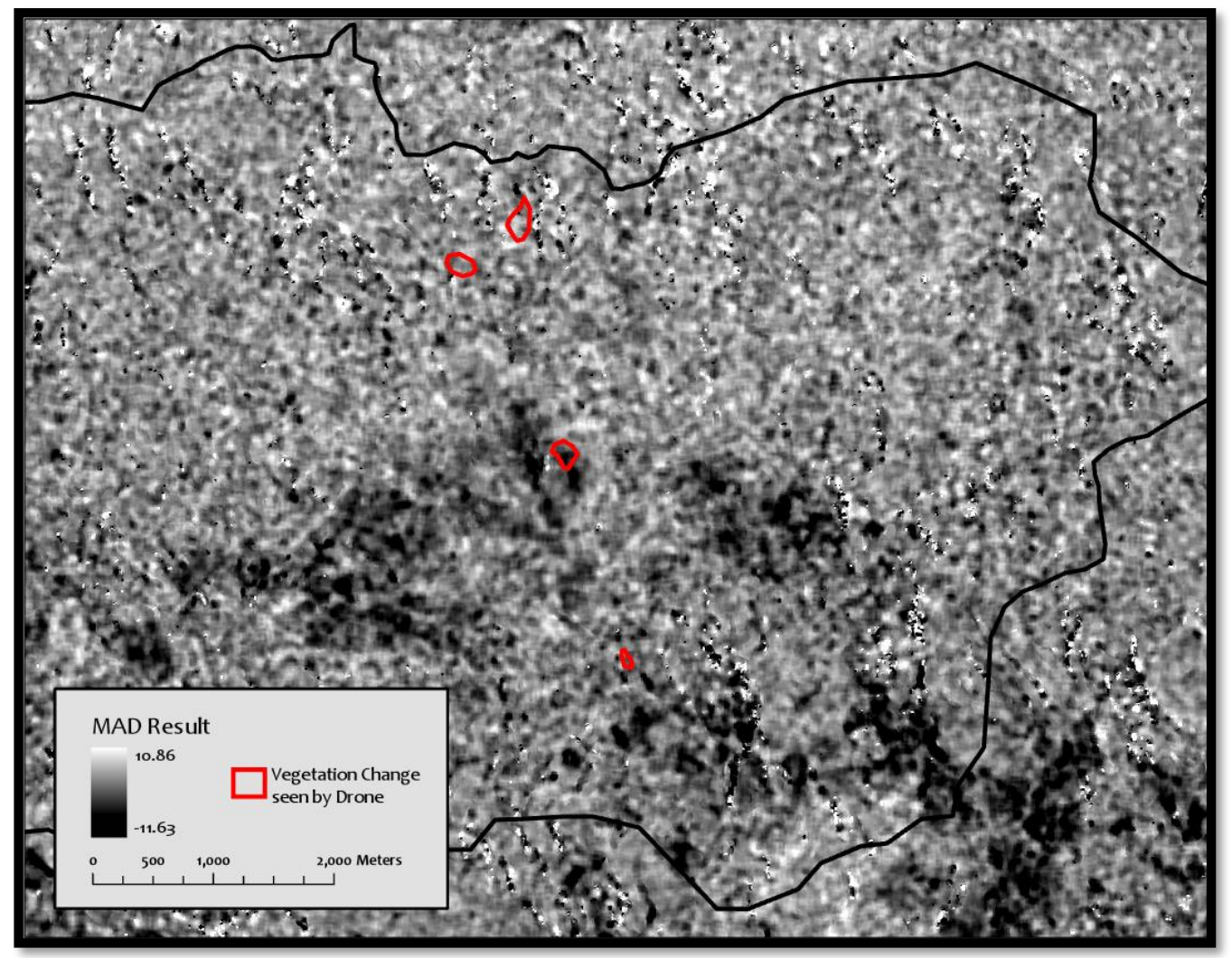

Figure 6-1: Raw result of MAD Analysis.

According to the theory of MAD analysis, values close to zero would indicate areas with no change. Pixels with high absolute values would indicate areas that had experienced change. In accordance with this idea, thresholds were applied to mask out areas with values close to zero, and group pixels to find areas where change had occurred. Figure 6-2 shows the result of one preliminary threshold pair applied to the MAD result. 


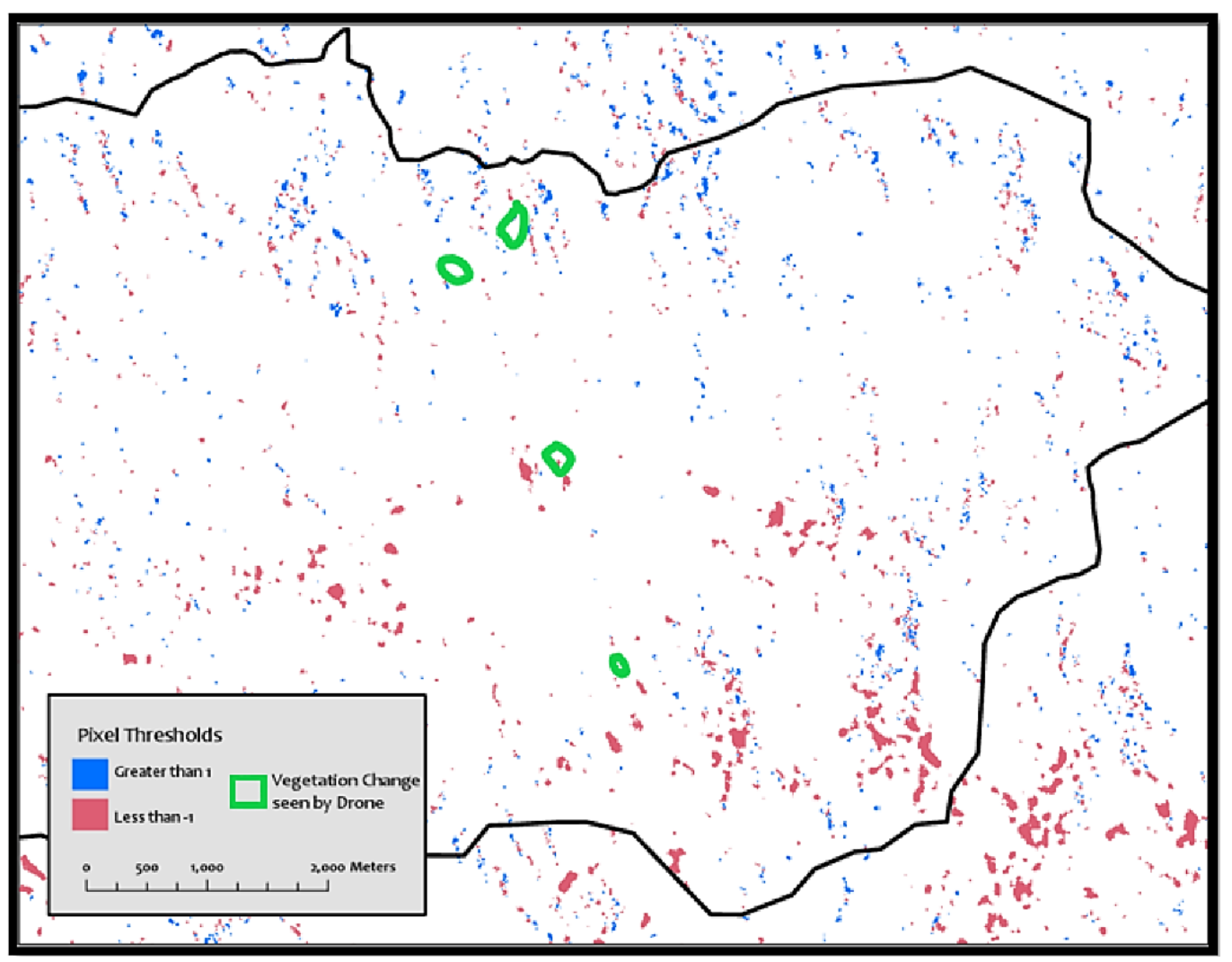

Figure 6-2: Example of threshold applied to MAD result.

Using the drone imagery as a guide, a final threshold pair of -.08 and .4 was created which showed the most discrete patterns that may overlap with the drone imagery polygons, shown in Figure 6-3. With this threshold, it was possible to see patterns and clustering of similar values within the valley. 


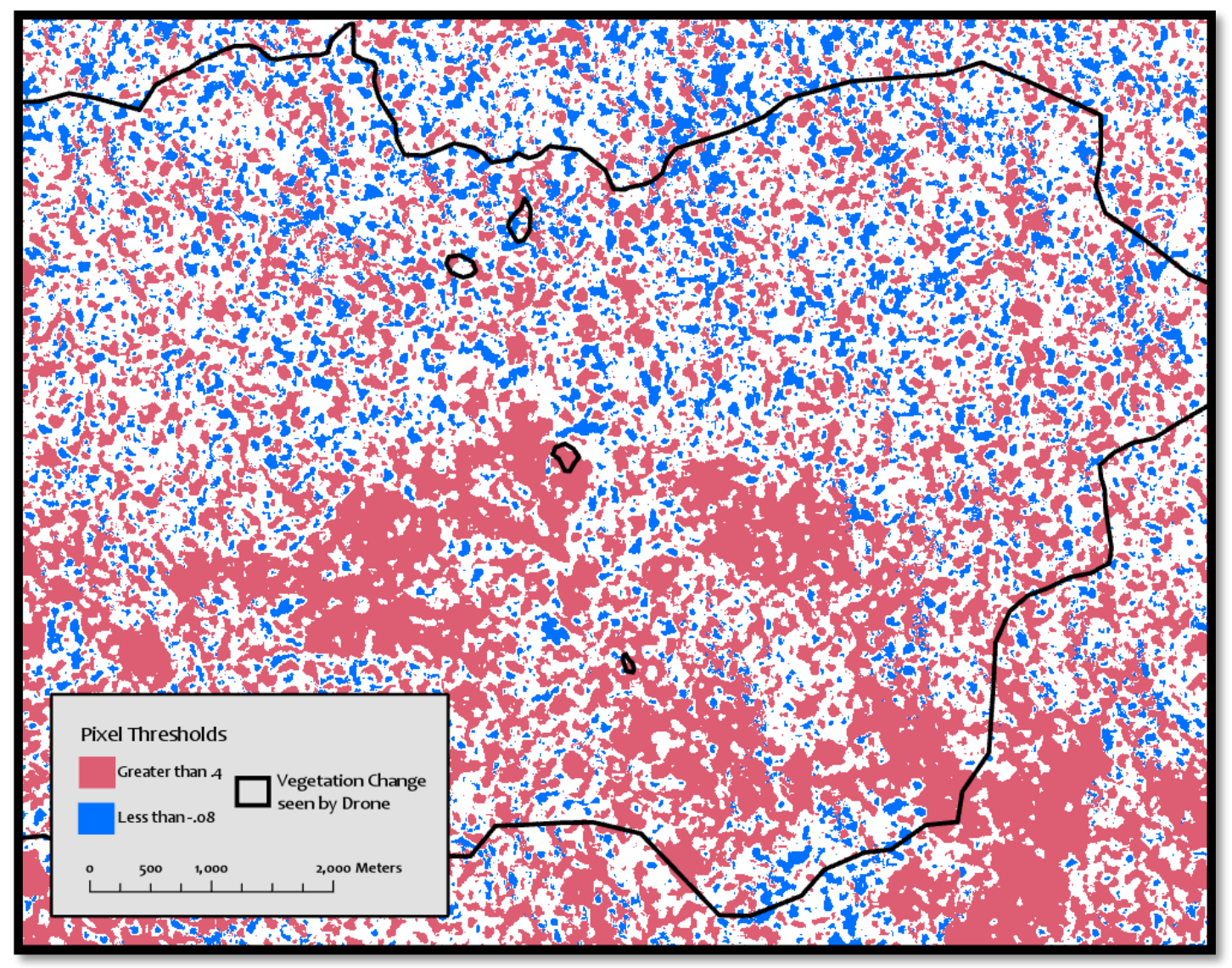

\section{Figure 6-3: Distribution of grouped pixel values based on most interpretable threshold pairs.}

In Figure 6-3, pixels with values greater than or equal to the high threshold are depicted in red, and pixels with values less than or equal to the low threshold are depicted in blue. When examining the polygons derived from drone imagery that denoted areas of vegetation loss, there was a stronger presence of red pixels, showing that there were large positive values in these areas. When examining the polygon which denoted vegetation growth, furthest northeast, red and blue pixels were both displayed, although blue was more prominent. This showed that there were large negative values within this area. However, taking a closer look at the control areas where drone imagery had indicated a definitive change between the study dates, depicted in Figure 6-4, there was not a conclusive pattern shown between changes indicated by drone imagery and changes indicated by SAR analysis.

Additionally, by looking closer at the drone imagery and MAD results, as in Figure 6-4, the MAD thresholds indicated change in areas where drone imagery showed that land cover type was consistent. This is concluded through the splotchy appearance. 

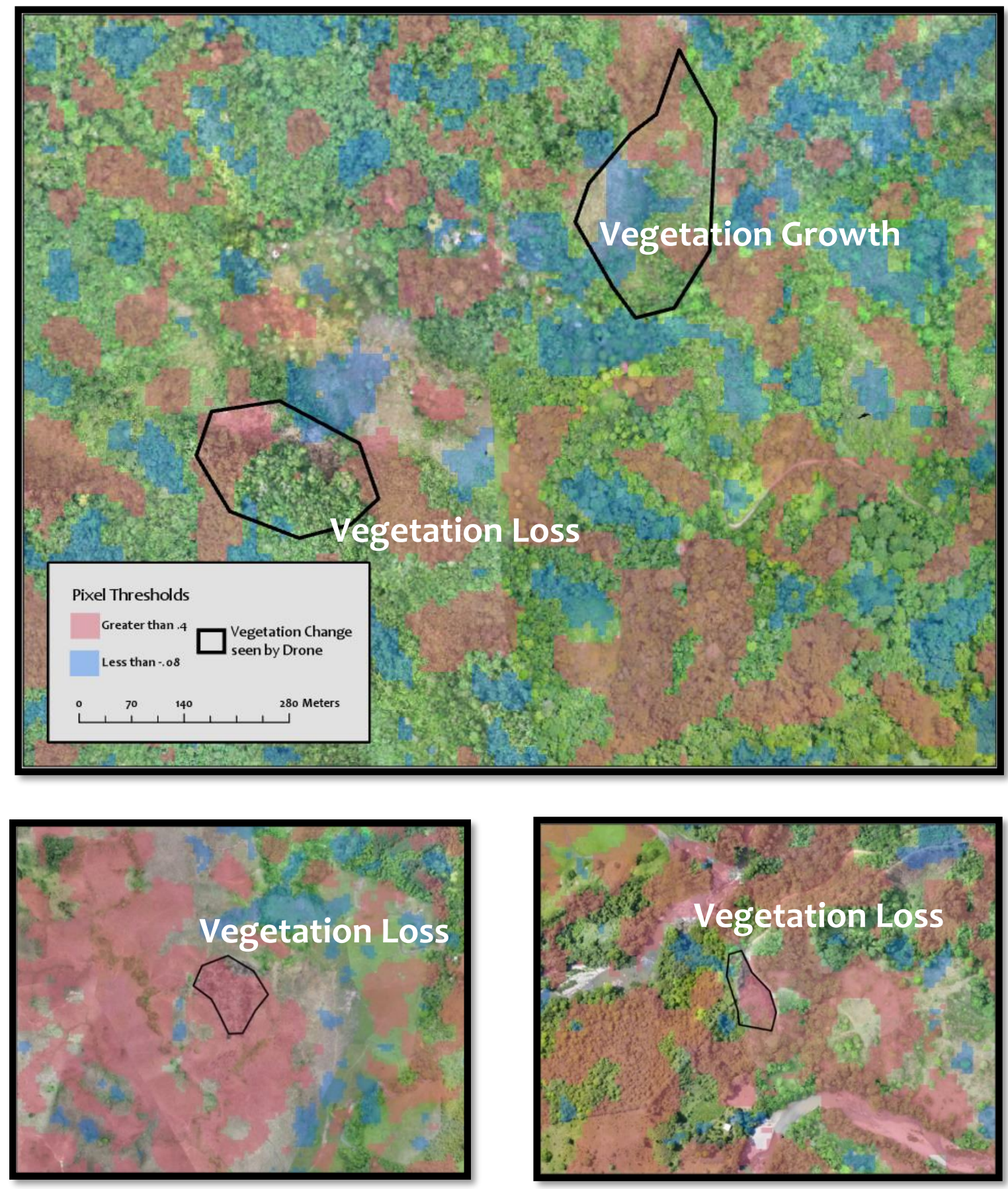

Figure 6-4: A closer look at the thresholded MAD result with 2019 drone imagery. 


\subsection{Change Detection with Principal Component Analysis}

Due to the similarity of MAD and Principal Component Analysis (PCA), the latter was implemented on the same original preprocessed SAR images to evaluate the performance of MAD. PCA can be used for change detection because it isolates the similarity from the difference between two measurements by projecting them into two perpendicular measurements, illustrated in Figure 6-5.

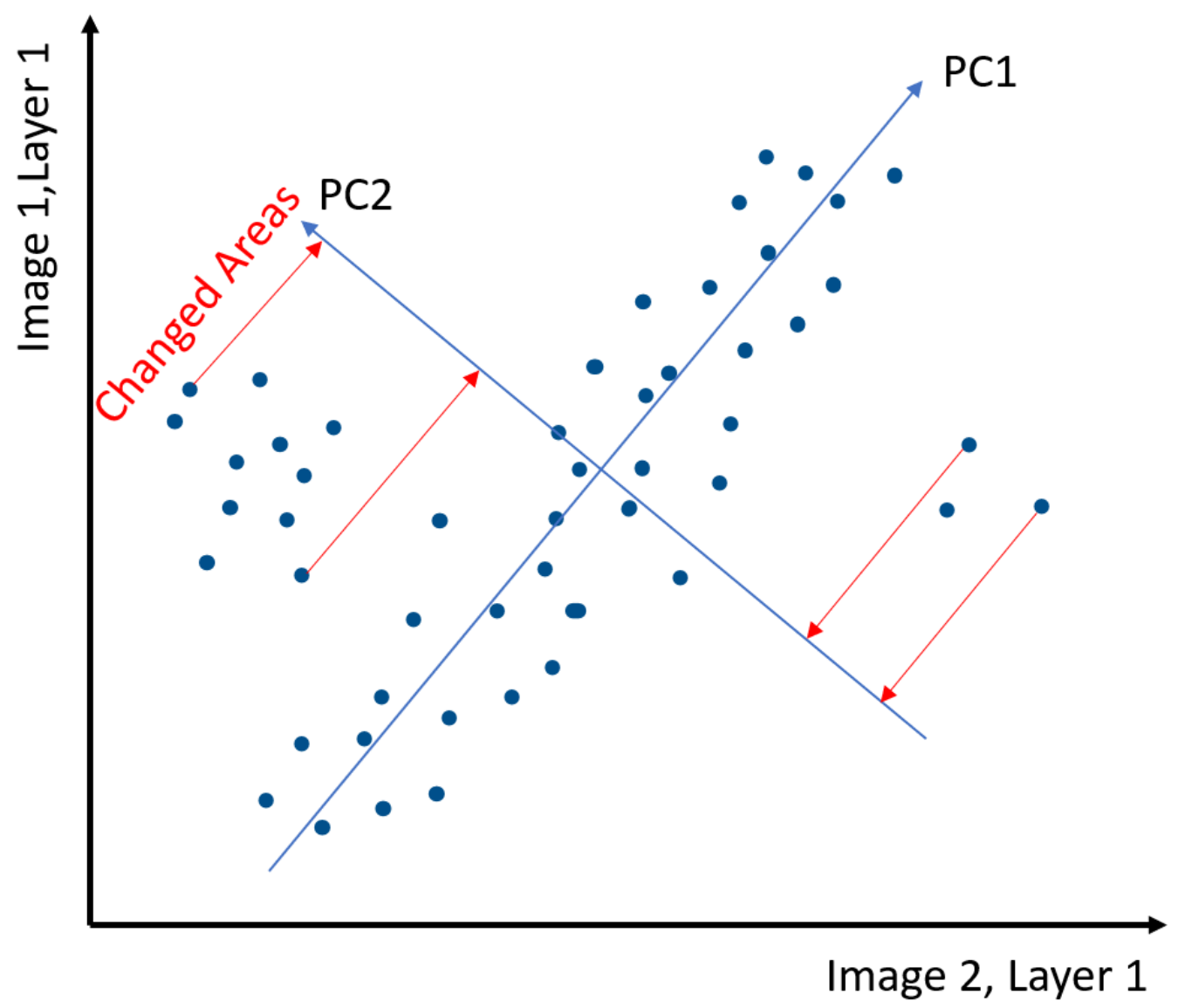

Figure 6-5: Scatter plot depicting principal components.

In Erdas Imagine 16.5, the VV and VH polarization layers from each year of the SAR image data were used to find changes from the Principal Component (PC) transformation. The following sections outline the results of these tests. 


\subsubsection{Principal Component of VV Polarization}

The first PC of the VV layers showed a wide variation in the pixel values, creating an image which looked clear, as shown in Figure 6-6.

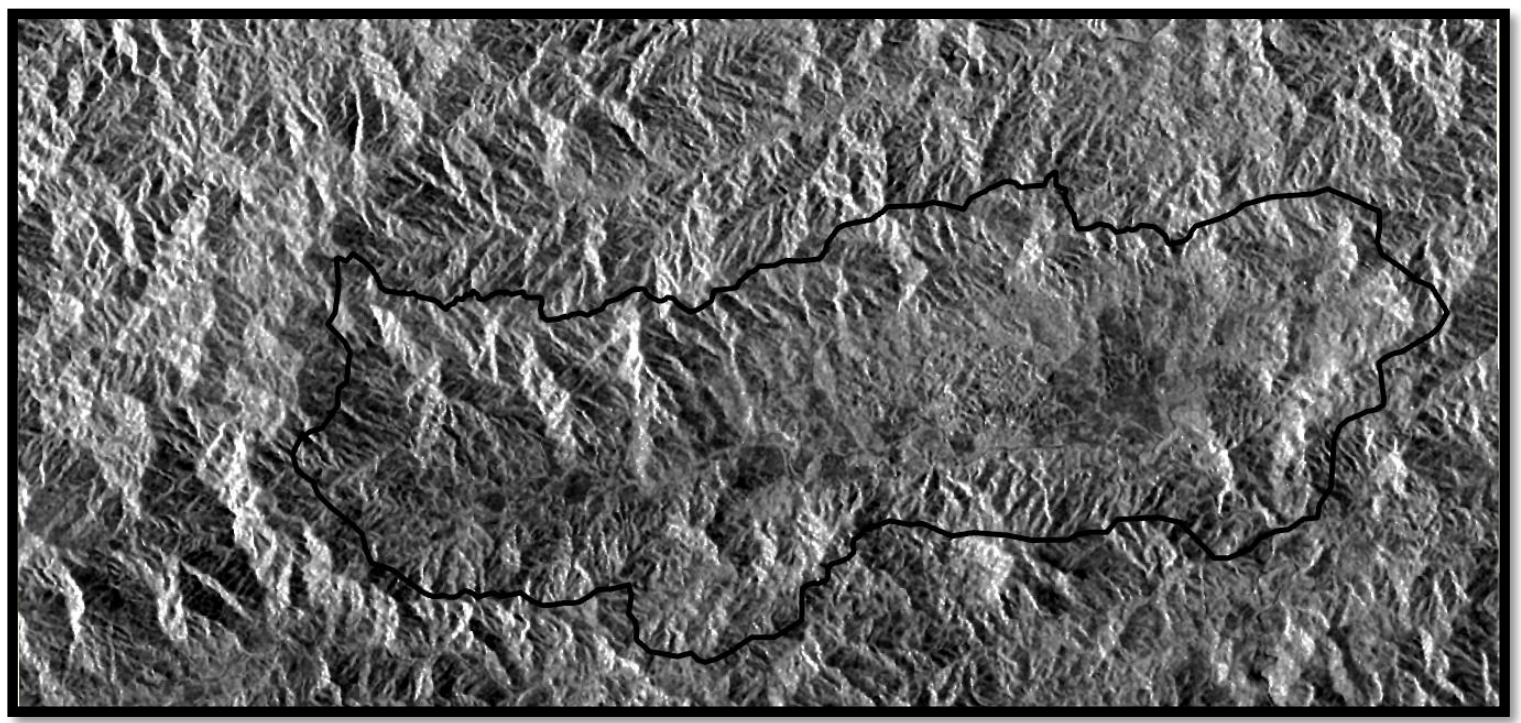

Figure 6-6: First layer of PCA result.

The second PC showed a smaller range of pixel values, pointing to areas where the two images were different. Pixel values of high absolute values in the second layer indicate change, similar to MAD analysis results. These results showed very similar patterns, shown in Figure 6-7. 

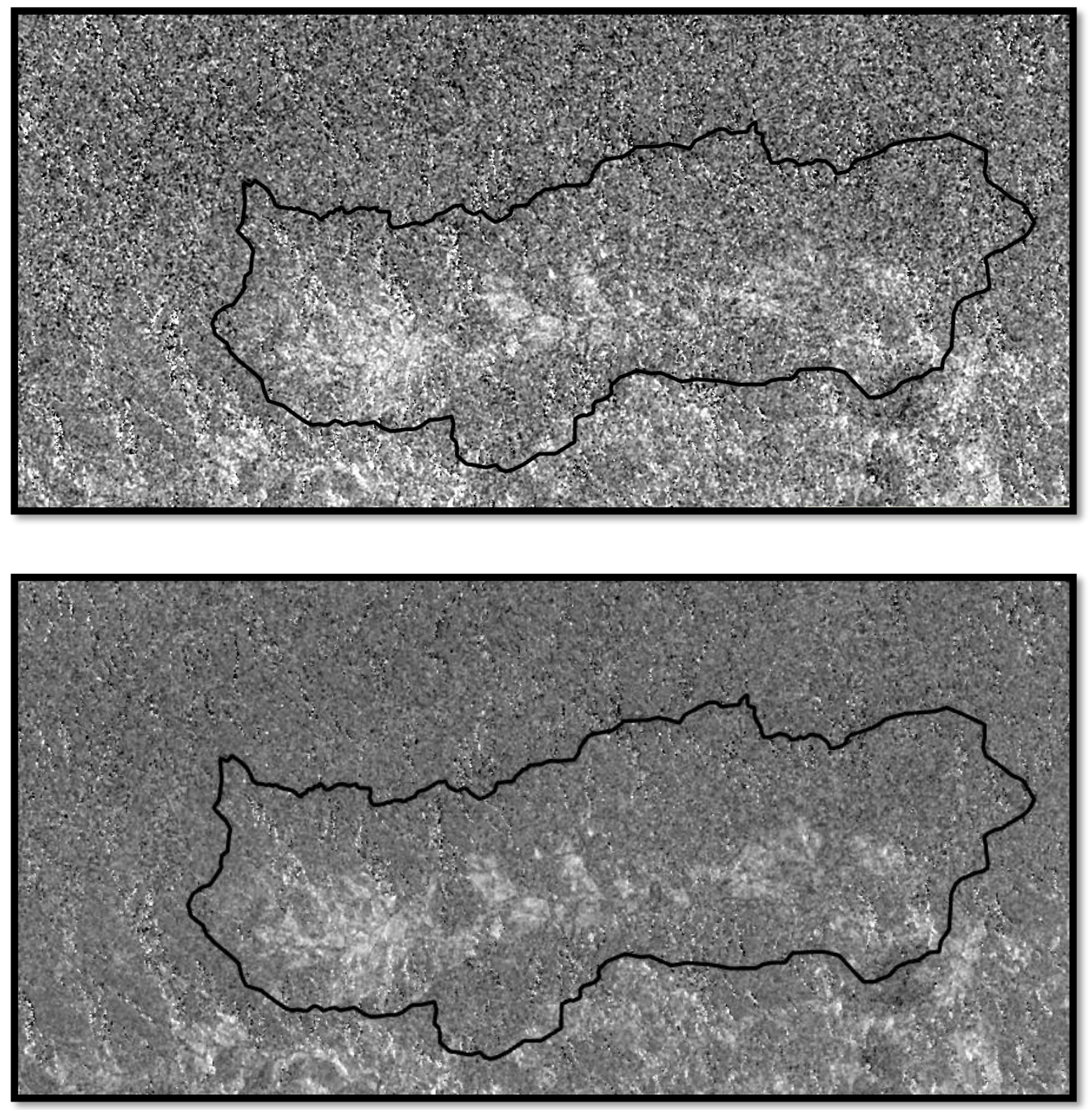

Figure 6-7: A comparison of the second VV PC (top) and MAD (bottom) layers used to detect multitemporal changes. The lightest and darkest colors represent high absolute values and indicate areas of change.

\subsubsection{Principal Component of VH Polarization}

The PCA process was implemented with the original VH layers to check for differences between the VV and VH layers as well as also comparing to the MAD result. In Figure 68 , the second layers of the VV and VH PCA result show minimal differences in the patterns of the resulting images. In these results, there are extreme high values and extreme low values speckled throughout the image, a result which was found after MAD as well. The MAD and PCA showed similar patterns in change detection. 

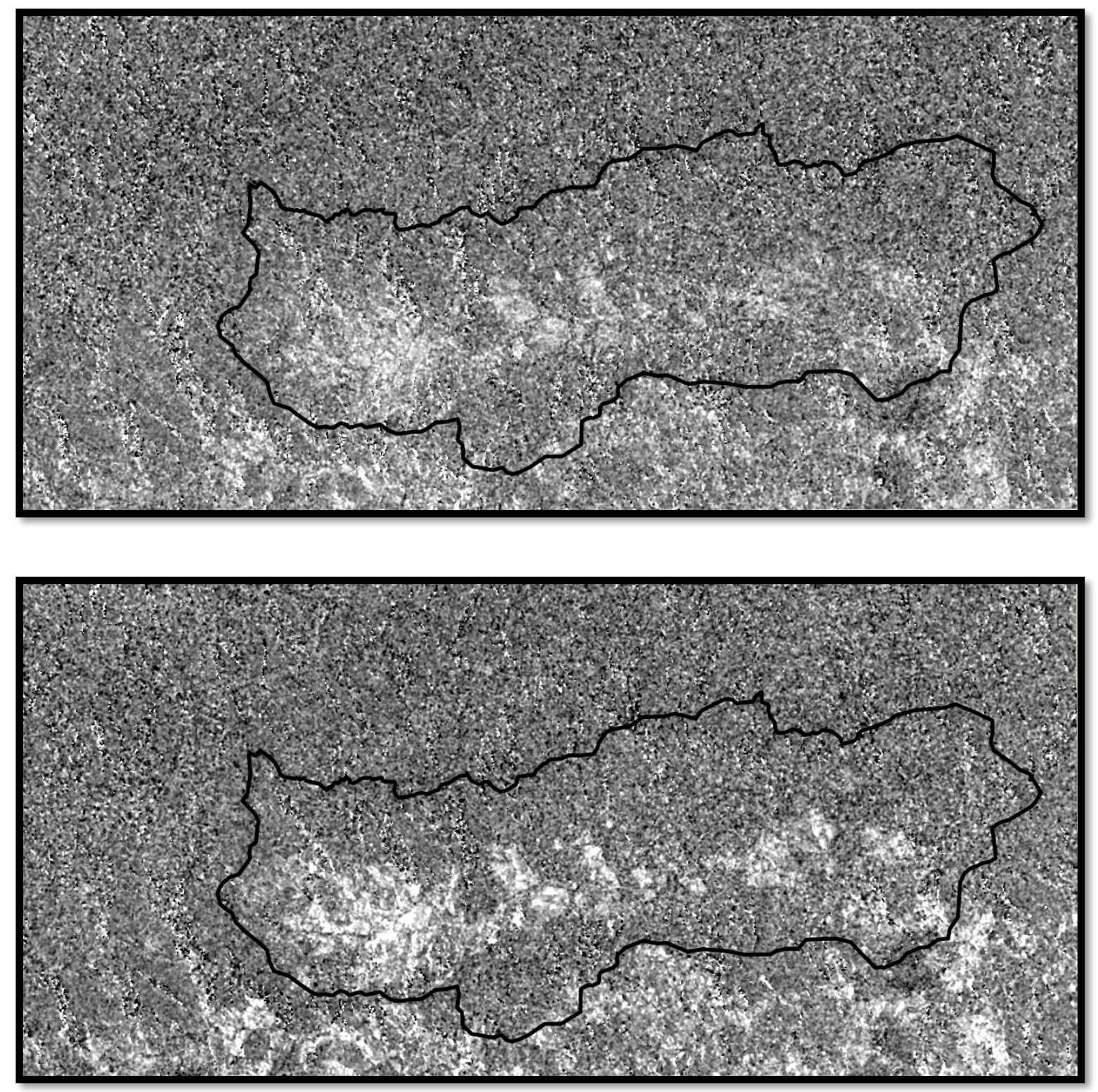

\section{Figure 6-8: Second Principal Components of PCA Analysis of VV layers (top) and VH layers (bottom).}

\subsubsection{Implications}

PCA and MAD showed similar results when implemented with the same SAR images. There was a similar pattern in detecting change, although the change may not reflect true land cover changes, as it does not match up with drone imagery. This may be due to GRD SAR data not reflecting explicitly through the intensity value. This comparison implies that the MAD and PCA work for change detection, though GRD SAR data may not be best suitable for the intended analyses. 


\subsection{Discussion}

By working with multiple datasets from different types of imagery, inconsistencies may have led to results with which distinct conclusions were not possible. These inconsistencies include those surrounding spatial resolution and georeferencing. In addition, the two types of data used, SAR and multispectral, inherently record different attributes about the land surface. While an objective of this project was to test the method by looking for correlation between the two types of data, additional work may be necessary to find conclusive information about the relationship.

\subsubsection{Spatial Resolution and Georeferencing}

After preprocessing and analysis, the spatial resolution of the SAR image data was approximately 9.87 meters. The approximate spatial resolution of the multispectral drone image data was .4 meters. This difference may be a cause of inconsistency between the distributions of results from the multispectral and SAR data. Additionally, pixels may extend across different geographic areas. This causes generalization of parcels, and if there were inaccuracies even after co-registering the two sets of SAR image data, the MAD analysis would find differences between the images where there may not have been in reality.

One example of a georeferencing issue with drone imagery showed how much of a difference there was between where the imagery was located on the map and where the displayed parcel of land was truly located on the earth. Figure 6-9 depicts this at one location. The 2018 image sits below the 2019 image, which can be noticed by looking at the edge of the bare patch northeast of the polygon.
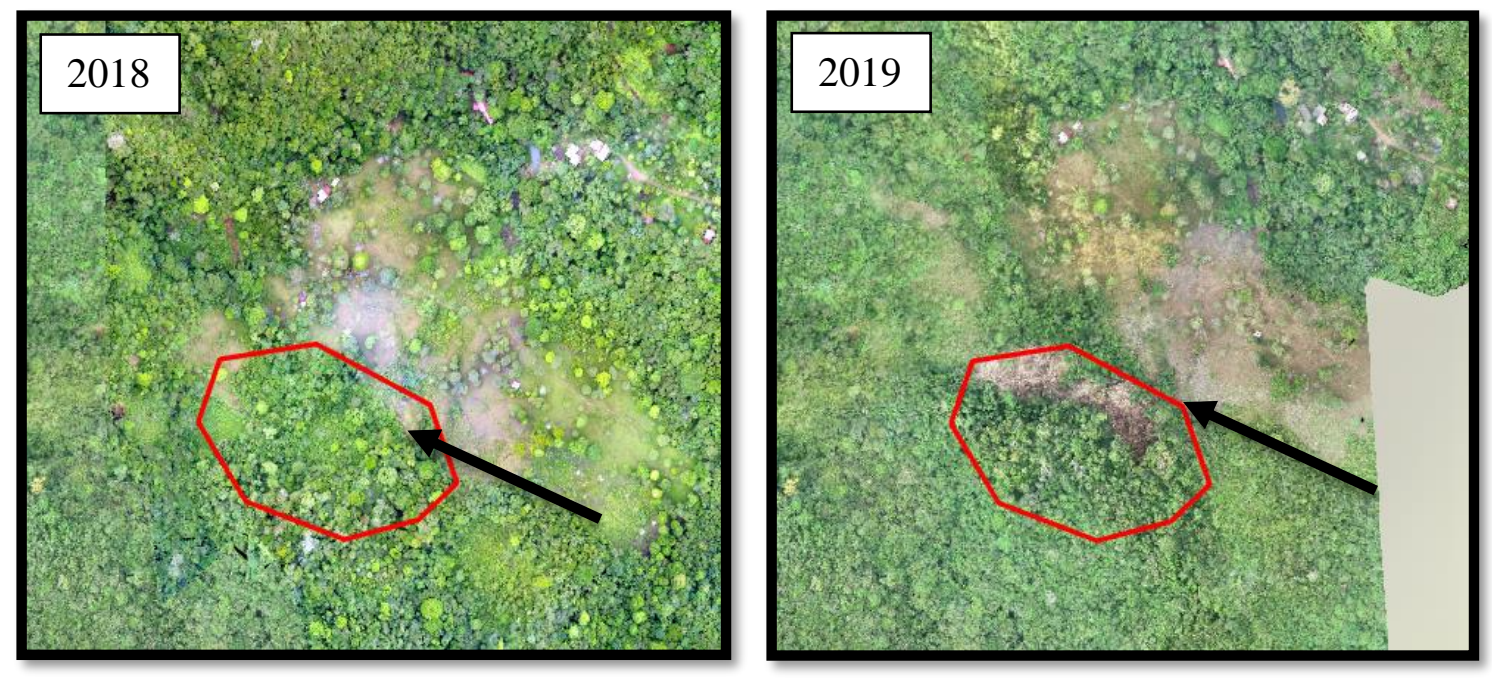

Figure 6-9: Inconsistency of georeferenced drone imagery. 


\subsubsection{Data Limitations}

The biggest data limitation that was faced in this project was concerning drone data. As discussed in Section 6.3.1, issues in georeferencing created some uncertainty. In addition to this issue, a relatively small geographic region had drone data from both years of 2018 and 2019. Furthermore, the areas in which a distinct and significant impact to the land had occurred were even smaller. Despite considerably concise spatial resolutions involved in the system, the result of the analysis showed that broader regions may be necessary to make conclusions about the relationships between multispectral drone imagery and SAR analysis data.

Since the SAR data used in this project only contained backscatter information about the returning radar wave, there was a limitation encountered at this step. This limitation was that there was an unknown regarding how backscatter would be affected by each surface type. The backscatter could be similar even in different vegetation types if the surface texture was relatively the same. Another limitation of SAR which can be present in many scenarios of satellite remote sensing is that changes in atmospheric characteristics can bend or otherwise affect the signal being recorded. While preprocessing aims to decrease the severity of these types of inconsistencies, there may still be issues when attempting to make small scale observations.

\subsection{Other Findings}

During interpretation of the SAR results, the general distribution and pattern of the valley showed extreme negative values heavily clustered in the central part of the valley, as seen in Figure 6-10. This part of the valley is the most developed and consistently managed for agriculture and grazing. This information led to the inference that the land cover of this region was consistent. Drone imagery from 2019 covered one large region, showing it to be pastureland. In Figure 6-10, this area is outlined in red. This region also correlates with high pixel values displayed in the MAD result. Unanticipated outcomes of the MAD analysis due to limitations or unexpected errors previously discussed, may have created an image in which areas of no change were not represented by pixel values close to zero. 


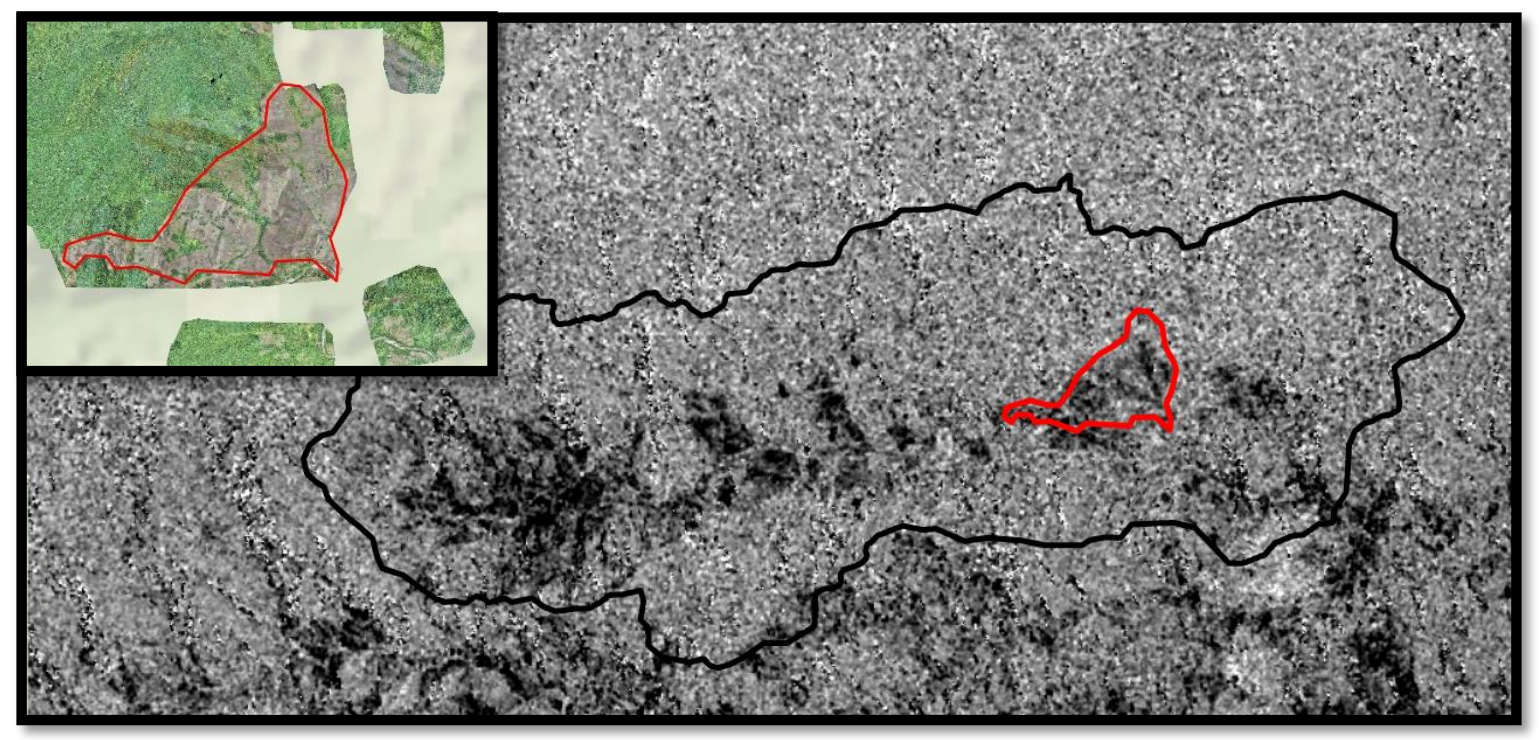

Figure 6-10: Overall pattern of Mamoní Valley and potential correlation between MAD result and developed areas depicted in drone imagery.

This result suggests that there may be a correlation between extreme negative values of the MAD result and developed regions of land.

\subsection{Summary}

This chapter detailed the results of this project in terms of the analysis itself and the successes and setbacks of the various components involved. This included the raw results of the change detection analysis compared to land cover changes seen by drone imagery, followed by interpretation and process of applying context to the results. The limitations that were found throughout the project were discussed, and finally, unanticipated findings of the analysis were addressed. The following chapter will expand upon the points made in this chapter, providing recommendations for future work and a final summary of the project as a whole. 



\section{Chapter 7 - Conclusions and Future Work}

This chapter aims to conclude the project and the overall outcomes of the work put into it and the derived products. In addition to the successes, it will assess the limitations that challenged the success of the various aspects of the project. Finally, there is a discussion about the areas where the project could be improved and built upon.

\subsection{Project Summary}

This project aimed to help Mamoní Valley Preserve by adapting a change detection methodology to ArcGIS Pro so that rapid and repeatable monitoring was possible. This goal of the project meant having three parts.

The first was finding a type of method and analysis that would be suitable for finding changes, specifically, in the study area in Panama. Research and this project showed how Synthetic Aperture Radar (SAR) imagery was the best option for detecting land cover attributes in humid tropical areas, due to its ability to penetrate clouds. Then, Multivariate Alteration Detection (MAD), which uses Canonical Correlation, was implemented because it can find areas that have the biggest difference in correlation from one date to the next. This was implemented as a script tool in ArcGIS Pro. The result showed areas where the difference of the correlation created negative and positive values, and patterns were seen through what was, in some cases, relatively noisy areas.

The second part of this project was to evaluate the accuracy of the MAD results. Drone imagery was used to do this as well as Principal Component Analysis (PCA). Within the area that had been covered by drone flights, there were examples of areas that had changed between the dates of May 2018 and May 2019. These images and areas provided evidence that the MAD analysis produced a depiction of areas that changed in land cover. Additionally, drone imagery provided the qualitative data that specified whether the change that was shown in the MAD result was vegetation loss or vegetation growth. However, when implementing PCA for change detection with the same imagery, similar results were found.

The last part of reaching the goal of this project was making the method accessible to Mamoní Valley Preserve. This meant creating a tool that would automate the analysis and be shown in a user-friendly interface that would require little to no experience or expertise in GIS or remote sensing. Two tools were created in the form of customized workflows. The first was in the SentiNel Applications (SNAP) toolbox, which provides a function to create and save customized data processing chains. The second was a Model Builder workflow in Esri's ArcGIS Pro software, which provided a user-friendly interface for the MAD analysis. This tool creates new layers in the ArcGIS Pro map viewer, which can be viewed simultaneously with future drone imagery.

\subsection{Future Work}

This project provided a groundwork for creating a change detection method with SAR imagery that could allow Mamoní Valley Preserve managers to rapidly and easily find 
where growth is occurring and where loss is occurring. However, further work should be done in order to solidify and narrow down the results from the MAD analysis alone.

This method would be optimized if drone imagery was not needed to confirm results every time the tool runs, and if drone imagery was not needed to specify, with certainty, the type of change that occurred. In the future, SAR MAD analyses should inform project managers on where to focus drone mapping sites so that more can be understood and validated from the MAD analysis.

The first type of areas that should be tested are the locations in the MAD result where negative combination difference values are present. While the inference of this project is that those values indicate areas of growth or close to no change, it must be confirmed before using the tool alone. Additionally, mapping these areas with drones would help to find whether the pixels containing positive values that are scattered throughout mostly negative pixel areas, are a result of noise or inconsistencies in the SAR image, or if they indicate small changes within the forest, such as single trees falling or being cut. Additional tests with preprocessing may also help to find the solution to this.

The second type of areas that should be testing with more drone imagery are the areas that contain positive pixel values. By checking these areas, the correlation between positive pixel values and the type of change (which is loss in the main example), may be validated. Potential locations for further drone mapping for method validation are shown in Figure 7-1. Lighter colors indicate highly positive pixel values and potential deforestation. Darker colors indicate highly negative pixel values and potential growth.

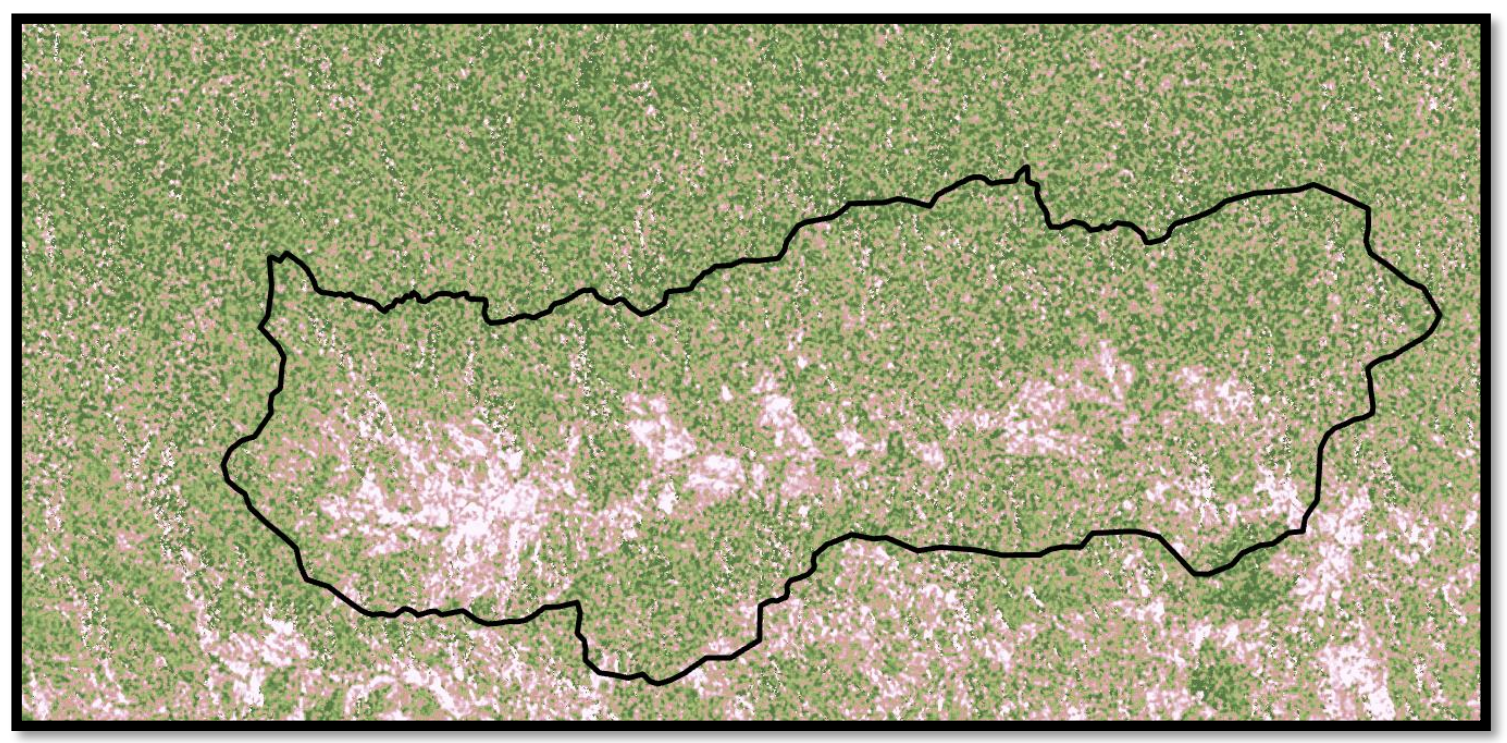

Figure 7-1: MAD result symbolized to show clustering of high and low values; potential future drone mapping sites.

Further research into SAR change detection methods would also be beneficial. Testing the MAD method against other methodologies, including those that use the complex SAR data such as Coherent Change Detection Processing (discussed in section 2.3.3), could speak further to the suitability of the method created in this project. 
On the tool automation part of this project, additional work to integrate preprocessing steps into ArcGIS Pro would be very valuable. By writing scripts to do each step of preprocessing, the preprocessing could be added to the MAD Model Builder and eventually allow the client to simply input two SAR products, run the tool, and get a result. 



\section{Works Cited}

Abdikan, S., Sanli, F. B., Ustuner, M., \& Calò, F. (2016). Land cover mapping using Sentinel-1 SAR data. The International Archives of Photogrammetry, Remote Sensing and Spatial Information Sciences, 41, 757.

Achard, F., Beuchle, R., Mayaux, P., Stibig, H.-J., Bodart, C., Brink, A., Carboni, S., Desclée, B., Donnay, F., Eva, H.D., Lupi, A., Raši, R., Seliger, R., \& Simonetti, D. (2014) "Determination of tropical deforestation rates and related carbon losses from 1990 to 2010." Global Change Biology, 20(8), 2540-2054.

Achard, F., Boschetti, L., Brown, S., Brady, M., DeFries, R., Grassi, G., ... \& Souza, C. (2014). "A sourcebook of methods and procedures for monitoring and reporting anthropogenic greenhouse gas emissions and removals associated with deforestation, gains and losses of carbon stocks in forests remaining forests, and forestation." (No. COP20-1). GOFC-GOLD.

Achard, F., Stibig, H. J., \& Eva, H. D. (2010) "Estimating tropical deforestation from Earth observation data." Carbon Management, 1(2), 271-287.

Adams, J. B., Smith, M. O., \& Gillespie, A. R. (1993) "Imaging Spectroscopy: interpretation based on spectral mixture analysis. In C. M. Pieters \& P. A. J. Englert, eds. Remote Geochemical Analysis Elemental and Mineralogical Composition." Cambridge University Press, 145-166.

Almeida-Filho, R., Rosenqvist, A., Shimabukuro, Y. E., \& Silva-Gomez, R. (2007) "Detecting deforestation with multitemporal L-band SAR imagery: a case study in western Brazilian Amazônia.” International Journal of Remote Sensing, 28(6), $1383-1390$.

Analytics Vidhya Content Team. (2016). Practical Guide to Principal Component Analysis (PCA) in R \& Python. Retrieved from https://www.analyticsvidhya.com/blog/2016/03/practical-guide-principalcomponent-analysis-python/.

Asner, G. P. (2001) "Cloud cover in Landsat observations of the Brazilian Amazon." International Journal of Remote Sensing, 22(18), 3855-3862.

Asner, G. P., Keller, M., Pereira, Jr, R., Zweede, J. C., \& Silva, J. N. M. (2004) "Canopy Damage and Recovery After Selective Logging in Amazonia: Field and Satellite Studies." Ecological Applications, 14(4), 280-298

Attema, E., Bargellini, P., Edwards, P. (2007). "Sentinel-1: The Radar Mission for GMES Operational Land and Sea Services". Bulletin. European Space Agency. 131, 1017.

Coates, A. G., Aubry, M. P., Berggren, W. A., Collins, L. S., \& Kunk, M. (2003). Early Neogene history of the Central American arc from Bocas del Toro, western Panama. Geological Society of America Bulletin, 115(3), 271-287.

Congalton, R. G., Gu, J., Yadav, K., Thenkabail, P., and Ozdogan M. (2014). “Global Land Cover Mapping: A Review and Uncertainty Analysis.” Remote Sensing, 6, 12070-12093.

De Sy, V., Herold, M., Achard, F., Asner, G. P., Held, A., Kellndorfer, J., \& Verbesselt, J. (2012). "Synergies of multiple remote sensing data sources for REDD+ monitoring." Current Opinion in Environmental Sustainability, 4(6), 696-706. 
Dobson, M. C., Pierce, L. E., \& Ulaby, F. T. (1996). Knowledge-based land-cover classification using ERS-1/JERS-1 SAR composites. IEEE Transactions on Geoscience and Remote Sensing, 34(1), 83-99.

Engineer's Journal. (2018). "Let's get things straight in aerial imagery." Retrieved from http://www.engineersjournal.ie/2018/06/26/lets-get-things-straight-aerialimaging/.

Erasmi, S. \& Twele, A. (2009) "Regional land cover mapping in the humid tropics using combined optical and SAR satellite data - a case study from Central Sulawesi, Indonesia." International Journal of Remote Sensing, 30(10), 2465-2478.

Esri. (n.d.) "What is raster data?" Retrieved from http://desktop.arcgis.com/en/arcmap/10.3/manage-data/raster-and-images/what-israster-data.htm.

European Space Agency. (n.d.-A) "Data Products." Retrieved from https://sentinel.esa.int/web/sentinel/missions/sentinel-1/data-products.

European Space Agency. (n.d.-B)."User Guides - Sentinel-1 SAR - Acquisition Modes". Retrieved from https://sentinel.esa.int/web/sentinel/user-guides/sentinel-1-sar.

European Space Agency. (2015). "Sentinel 1 Data Access and Products". Retrieved from https://sentinel.esa.int/web/sentinel/user-guides/sentinel-1-sar.

European Space Agency Sentinel Applications Platform. (n.d.) “Geometric.” Sentinel Applications Platform Toolbox Help Document.

European Space Agency Sentinel Online. (n.d.) "Sentinel-1". Retrieved from https://sentinel.esa.int/web/sentinel/missions/sentinel-1.

FAO (2011) "State of the World's Forests 2011." Rome: Food and Agriculture Organization of the United Nations.

Friedl, M. A., Sulla-Menashe, D., Tan, B., Schneider, A., Ramankutty, N., Sibley, A., Huang, X. (2010). "MODIS Collection 5 global land cover: Algorithm refinements and characterization of new datasets." Remote Sensing of Environment, 114(1),168-182.

Geoversity. (2019). Retrieved from https://geoversity.org/en.

Ghazoul, J. \& Sheil, D. (2010) “Tropical Rain Forest Ecology, Diversity, and Conservation." Oxford: Oxford University Press.

Gullison, R. E., Frumhoff, P. C., Canadell, J.G., Field, C.B., C,D., Hayhoe, K., Avissar, R., Curran, L.M., Friedlingstein, P., \& D,C. (2007) "Tropical Forests and Climate Policy." Science, 316(5827), 985-986.

Hammer, D., Kraft, R., \& Wheeler, D. (2009). Forma: Forest monitoring for action-rapid identification of pan-tropical deforestation using moderate-resolution remotely sensed data. Center for Global Development Working Paper, (192).

Hammer, D., Kraft, R., \& Wheeler, D. (2014) "Alerts of forest disturbance from MODIS imagery." International Journal of Applied Earth Observation and Geoinformation, 33, 1-9.

Herold, M. (2009). "An assessment of national forest monitoring capabilities in tropical non-Annex I countries: Recommendations for capacity building." Retrieved from https://redd.unfccc.int/uploads/2_153_redd_20090710_norway.pdf.

Hoan, N. T., Tateishi, R., Alsaaideh, B., Ngigi, T., Alimuddin, I., \& Johnson, B. (2013) "Tropical forest mapping using a combination of optical and microwave data of ALOS.” International Journal of Remote Sensing, 34(1), 139-153. 
Kuplich, T. M. (2006) "Classifying regenerating forest stages in Amazônia using remotely sensed images and a neural network." Forest Ecology and Management, 234, 1-9.

Lehmann, E., Caccetta, P., Zhou, Z. S., Mitchell, A., Tapley, I., Milne, A., ... \& McNeill, S. (2011, April). Forest discrimination analysis of combined Landsat and ALOSPALSAR data. In Proceedings of International Symposium on Remote Sensing of Environment, Sydney, Australia (Vol. 1015).

Lehmann, E. A., Wallace, J. F., Caccetta, P. A., Furby, S. L., \& Zdunic, K. (2013) "Forest cover trends from time series Landsat data for the Australian continent." International Journal of Applied Earth Observation and Geoinformation, 21, 453- 462.

Lu, D., Li, G., \& Moran, E. (2014) "Current situation and needs of change detection techniques." International Journal of Image and Data Fusion, 5(1), 13-38.

Malenovský, Z., Rott, H., Cihlar, J., Schaepman, M. E., García-Santos, G., Fernandes, R., \& Berger, M. (2012). "Sentinels for science: Potential of Sentinel-1,-2, and-3 missions for scientific observations of ocean, cryosphere, and land." Remote Sensing of Environment, 120, 91-101.

Nielsen, A. A., Conradsen, K., \& Simpson, J. J. (1998). Multivariate alteration detection (MAD) and MAF postprocessing in multispectral, bitemporal image data: New approaches to change detection studies. Remote Sensing of Environment, 64(1), 119.

Oestreicher, J. S., Benessaiah, K., Ruiz-Jaen, M. C., Sloan, S., Turner, K., Pelletier, J., ... \& Potvin, C. (2009). Avoiding deforestation in Panamanian protected areas: an analysis of protection effectiveness and implications for reducing emissions from deforestation and forest degradation. Global Environmental Change, 19(2), 279291.

Pan, Y., Birdsey, R. A., Fang, J., Houghton, R., Kauppi,P. E., A,W., Phillips, O. L., Shvidenko, A., Lewis, S. L., Canadell, J. G., Ciais, P., Jackson, R. B., Pacala, S., Mcguire, A. D., Piao, S., Rautiainen, A., \& Hayes, D. (2011) “A Large and Persistent Carbon Sink in the World's Forests." Science, 333(6045), 988-993.

Preiss, M., \& Stacy, N. J. (2006). Coherent change detection: Theoretical description and experimental results (No. DSTO-TR-1851). DEFENCE SCIENCE AND TECHNOLOGY ORGANISATION EDINBURGH (AUSTRALIA).

Rainforest Trust. (2017). "Climate Change Series Part 1 - Rainforests Absorb, Store Large Quantities of Carbon Dioxide." Retrieved from https://www.rainforesttrust.org/climate-change-series-part-1-rainforests-absorbstore-large-quantities-carbon-dioxide/

Reiche, J., Verbesselt, J., Hoekman, D., \& Herold, M. (2015). "Fusing Landsat and SAR time series to detect deforestation in the tropics." Remote Sensing of Environment, 156, 276-293.

Rignot, E., Salas, W., \& Skole, D. L. (1997) "Mapping deforestation and secondary growth in Rondonia, Brazil, using imaging radar and thematic mapper data." Remote Sensing of Environment, 59(2), 167-179.

Ringnér, M. (2008). "What is principal component analysis?" Nature biotechnology, 26(3), 303. 
Sasaki, N., Asner, G. P., Knorr, W., Durst, P. B., Priyadi, H. R., \& Putz, F. E. (2011) "Approaches to classifying and restoring degraded tropical forests for the anticipated REDD+ climate change mitigation mechanism." Biogeosciences and Forestry, 4(1), 1-6.

Shimabukuro, Y. E., Duarte, V., Anderson, L. O., Valeriano, D. M., Arai, E., Freitas, R. M. De, Rudorff, B. F. T., \& Moreira, A. A. (2006) "Near real-time detection of deforestation in the Brazilian Amazon using MODIS imagery." An Interdisciplinary Journal of Applied Science, 1(1), 37-47.

Smith, B. T., Amei, A., \& Klicka, J. (2012). Evaluating the role of contracting and expanding rainforest in initiating cycles of speciation across the Isthmus of Panama. Proceedings of the Royal Society B: Biological Sciences, 279(1742), 3520-3526.

Souza, C. (2003) "Mapping forest degradation in the Eastern Amazon from SPOT 4 through spectral mixture models." Remote Sensing of Environment, 87(4), 494506.

Souza, C., Roberts, D., \& Cochrane, M. (2005) "Combining spectral and spatial information to map canopy damage from selective logging and forest fires." Remote Sensing of Environment, 98(2-3), 329-343.

Souza, C., Siqueira, J., Sales, M., Fonseca, A., Ribeiro, J., Numata, I., Cochrane, M., Barber, C., Roberts, D., \& Barlow, J. (2013) "Ten-Year Landsat Classification of Deforestation and Forest Degradation in the Brazilian Amazon." Remote Sensing, 5(11), 5493-5513.

Stern, N. (2007) "The economics of climate change." Cambridge, United Kingdom: Cambridge University Press.

Tyukavina, A., Stehman, S. V, Potapov, P. V, Turubanova, S., Baccini, A., Goetz, S. J., Laporte, N. T., Houghton, R. a, \& Hansen, M. C. (2013) "National-scale estimation of gross forest aboveground carbon loss: a case study of the Democratic Republic of the Congo.” Environmental Research Letters, 8(4), 044039.

Vaglio Laurin, G., Liesenberg, V., Chen, Q., Guerriero, L., Del Frate, F., Bartolini, A., Coomes, D., Wilebore, B., Lindsell, J., \& Valentini, R. (2013) “Optical and SAR sensor synergies for forest and land cover mapping in a tropical site in West Africa." International Journal of Applied Earth Observation and Geoinformation, $21,7-16$.

Van der Werf, G. R., Morton, D. C., DeFries, R. S., Olivier, J. G. J., Kasibhatla, P. S., Jackson, R. B., Collatz, G. J., \& Randerson, J. T. (2009) "CO2 emissions from forest loss." Nature Geoscience, 2(11), 737-738.

Walker, W. S., Stickler, C. M., Kellndorfer, J. M., Kirsch, K. M., \& Nepstad, D. C. (2010) "Large-Area Classification and Mapping of Forest and Land Cover in the Brazilian Amazon: A Comparative Analysis of ALOS/PALSAR and Landsat Data Sources." IEEE Journal of Selected Topics in Applied Earth Observations and Remote Sensing, 3(4), 594-604.

Webb, S. D., \& Stehli, F. G. (Eds.). (1985). The great American biotic interchange (No. 56: 57 (7/8) GRE). Plenum Press. 
Wheeler, D., Hammer, D., Kraft, R., \& Steele, A. (2014). Satellite-based forest clearing detection in the Brazilian Amazon: FORMA, DETER, and PRODES. World Resources Institute: Washington, DC, USA.

Zhang,J. (2010) "Multi-source remote sensing data fusion: status and trends." International Journal of Image and Data Fusion, 1(1), 5-24. 



\section{Appendix A. MAD Script in Python}

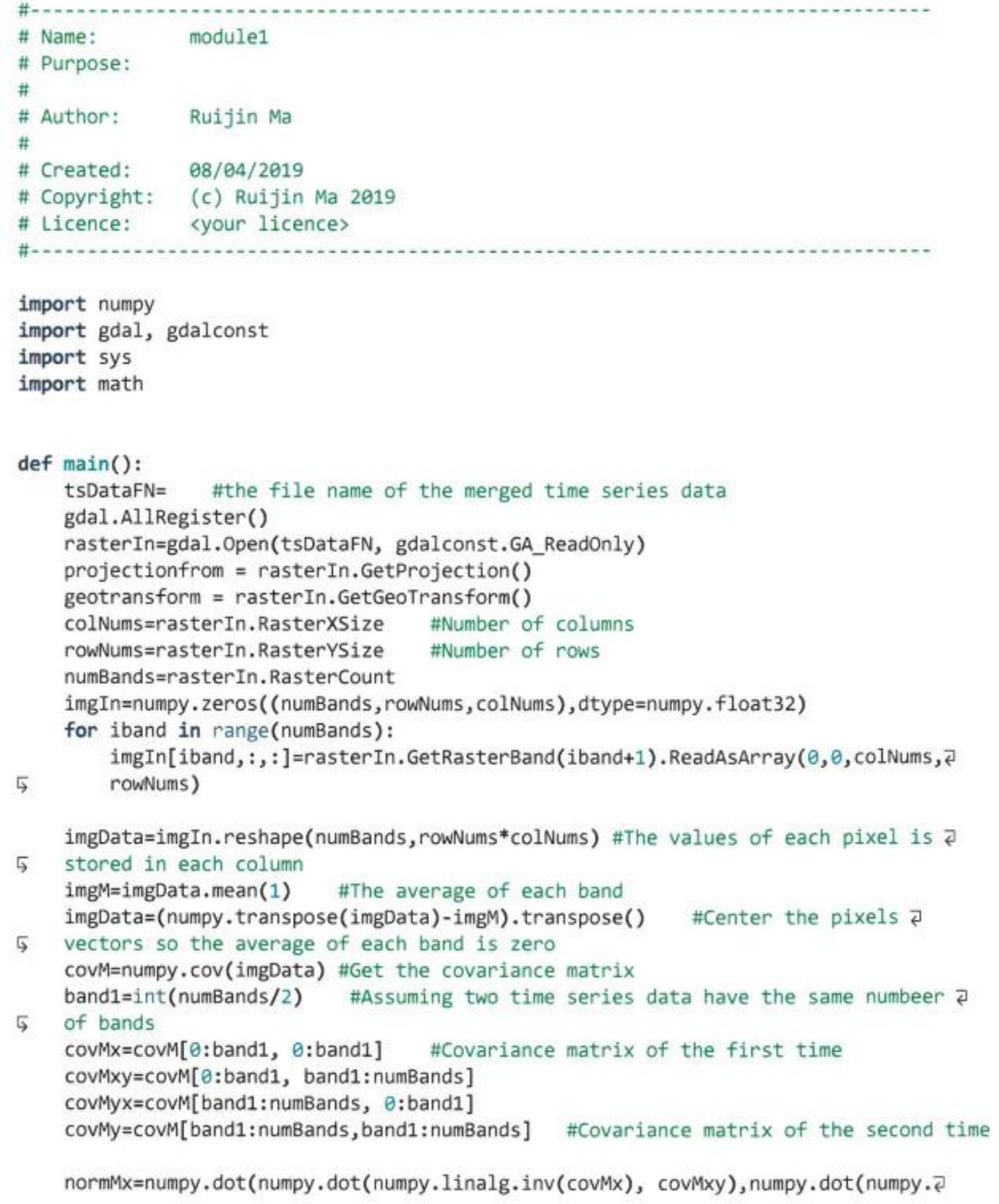


linalg.inv((covMy), $\operatorname{covMyx})$ )

normMy=numpy. $\operatorname{dot}$ (numpy. $\operatorname{dot}$ (numpy. linalg. inv(covMy), covMyx), numpy. $\operatorname{dot}$ (numpy.

5 linalg.inv (covMx), covMxy))

eigVax, eigVecx=numpy. linalg.eig (normMx)

eigVay, eigVecy=numpy.linalg.eig(normMy)

stdMx=numpy. $\operatorname{dot}($ eigVecx.transpose (), numpy. $\operatorname{dot}(\operatorname{cov} M x, e i g V e c x))$

stdMy=numpy. $\operatorname{dot}($ eigVecy.transpose (), numpy.dot (covMy, eigVecy))

$u x, s x, v x=n u m p y$. linalg.svd( $\operatorname{std} M x)$

uy, sy, vy=numpy.linalg.svd(stdMy)

invHX=numpy. linalg.inv (numpy. sqrt (numpy.diag $(s x))$ )

invHy=numpy. linalg.inv (numpy.sqrt (numpy.diag(sy)))

$s t d X=n u m p y \cdot \operatorname{dot}(n u m p y, \operatorname{dot}(u x$, invHx), vx)

stdY=numpy $\cdot \operatorname{dot}$ (numpy. dot (uy, invHy), vy)

$c c X=$ numpy $\cdot \operatorname{dot}($ numpy $\cdot \operatorname{dot}(\operatorname{stdX}$, eigVecx.transpose ()), imgData[ $[0:$ band $1,:]$ )

$c c Y=$ numpy. $\operatorname{dot}$ (numpy. dot (stdY, eigVecy.transpose()), imgData[band1: numBands, : ])

madData $=\mathrm{ccX}-\mathrm{ccY}$

madMat=madData. reshape(band1, rowNums, colNums) \#Re-arrange the pixels into an

5 image format

\#The next step is to perform a MAF process for change analysis

\#The MAD data is already independent, so its covariance matrix is technically $?$

5 a diagnal one

covMad=numpy. $\operatorname{cov}($ madData)

madData1=numpy. zeros (madData. shape, dtype=numpy. float)

for iband in range(band1):

madData1 [iband,: ] =madData[ [iband,: ]/math.sqrt (covMad [iband,iband])

\#madDatal is rescaled from madData to have unit variance per MAF requirement madMat1=madData1. reshape (band1, rowNums, colNums)

lagH=numpy $\cdot$ zeros (madMat 1 . shape, dtype=numpy. float)

lagV=numpy.zeros (madMat1. shape, dtype=numpy. float)

lagH $[:,:, \theta:$ colNums -1$]=$ madMat $1[:,:, 1:$ colNums $]$

$\operatorname{lagH}[:,:, \operatorname{col}$ Nums -1$]=\operatorname{madMat} 1[:,:, \theta]$

$\operatorname{lagV}[:, \theta:$ rowNums $-1,:]=$ madMat $1[:, 1:$ rowNums, : ]

lagH $[:$, rownums -1$]=\operatorname{madMat} 1[:, 0,:]$

difH=(madMat1-lagH) , reshape (band1, rowNums* colNums)

difV $=($ madMat1 1 lagV $)$. reshape (band1, rowNums*colNums)

$\mathrm{covH}=$ numpy. $\operatorname{cov}(\mathrm{difH})$

$\operatorname{covV}=$ numpy. $\operatorname{cov}(\mathrm{difV})$

lagCov= COVH + CovV

eigVL, eigVecL=numpy. linalg.eig(lagCov)

mafData=numpy.dot (eigVecL.transpose(), madData1)

mafMat=mafData. reshape (band1, rowNums, colNums)

outFN=tsDataFN.split( $\left.{ }^{\prime} .{ }^{\prime}\right)[\theta]+"$ mad.tif"

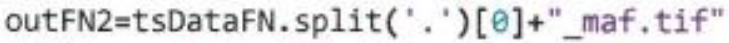

\#Write out the MAD data 
99

100

101

102

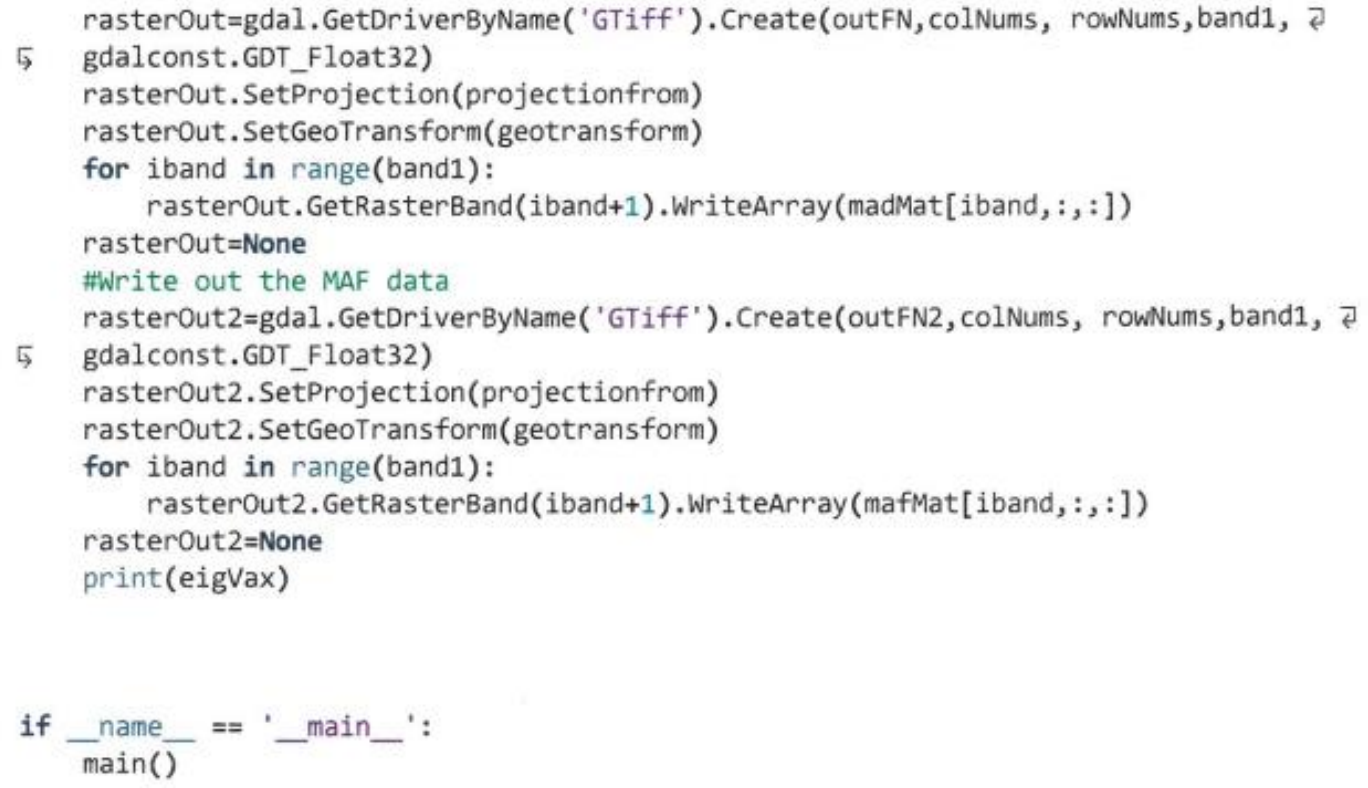




\section{Appendix B. Preprocessing Script in .XML}

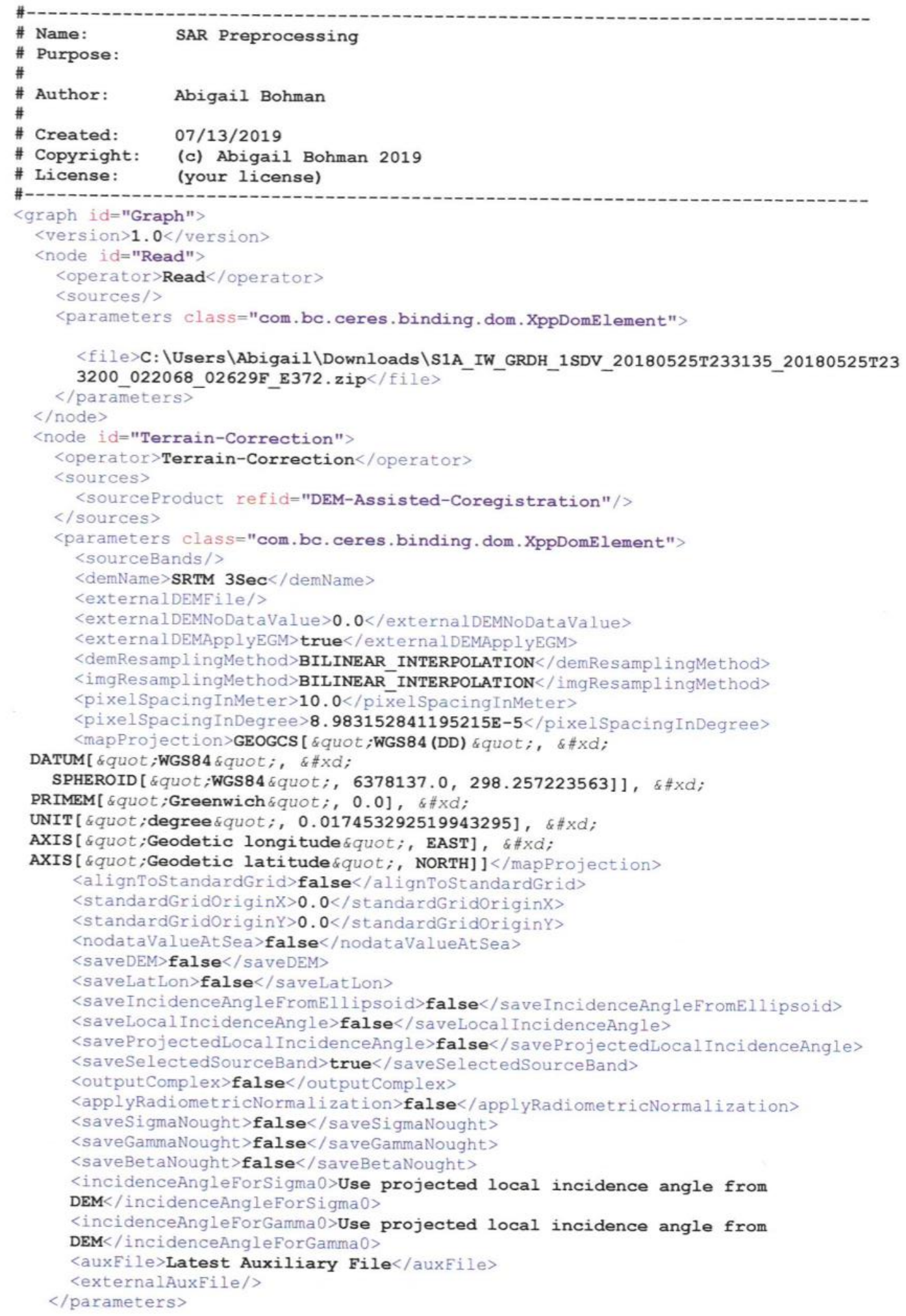




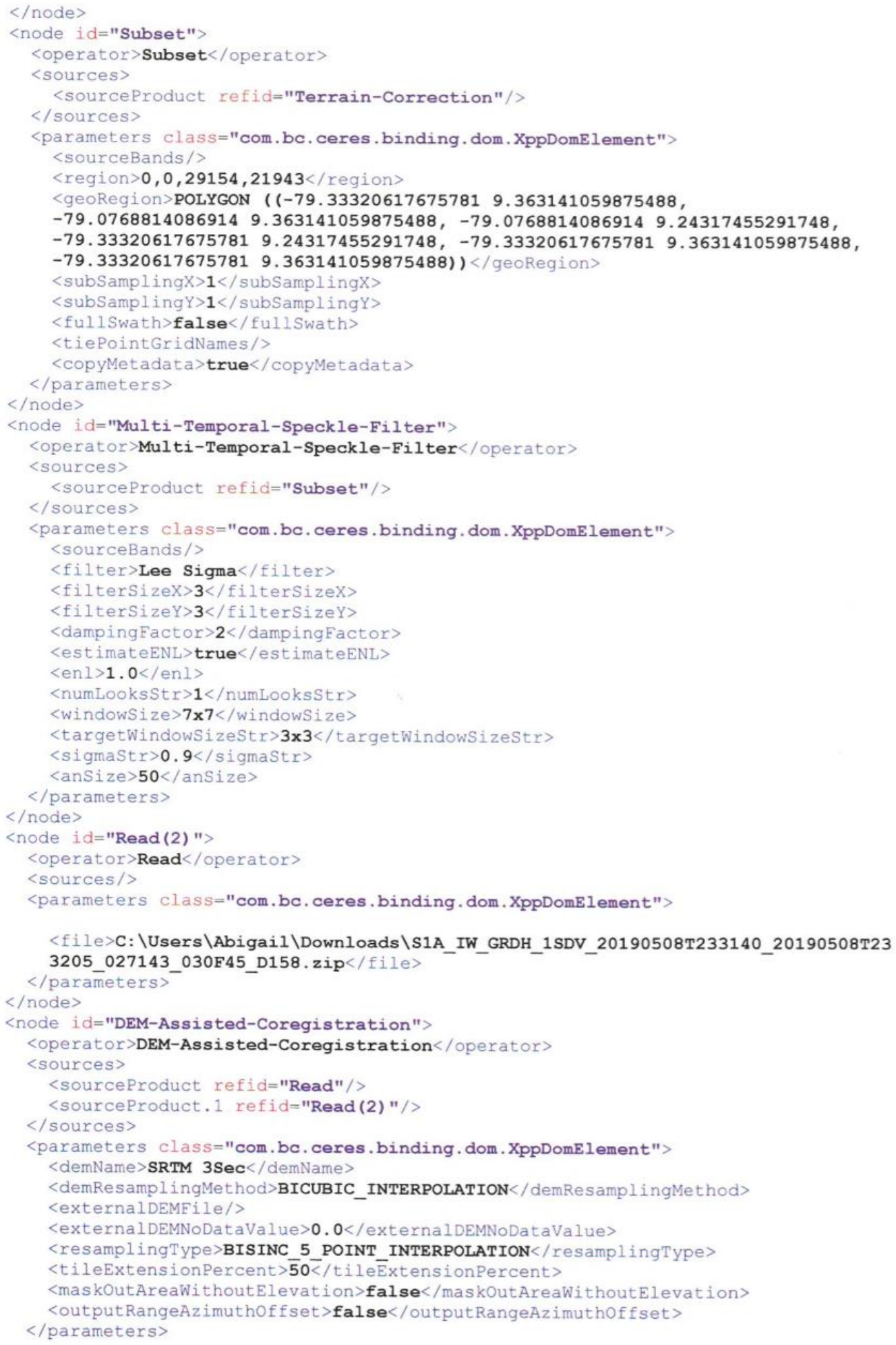




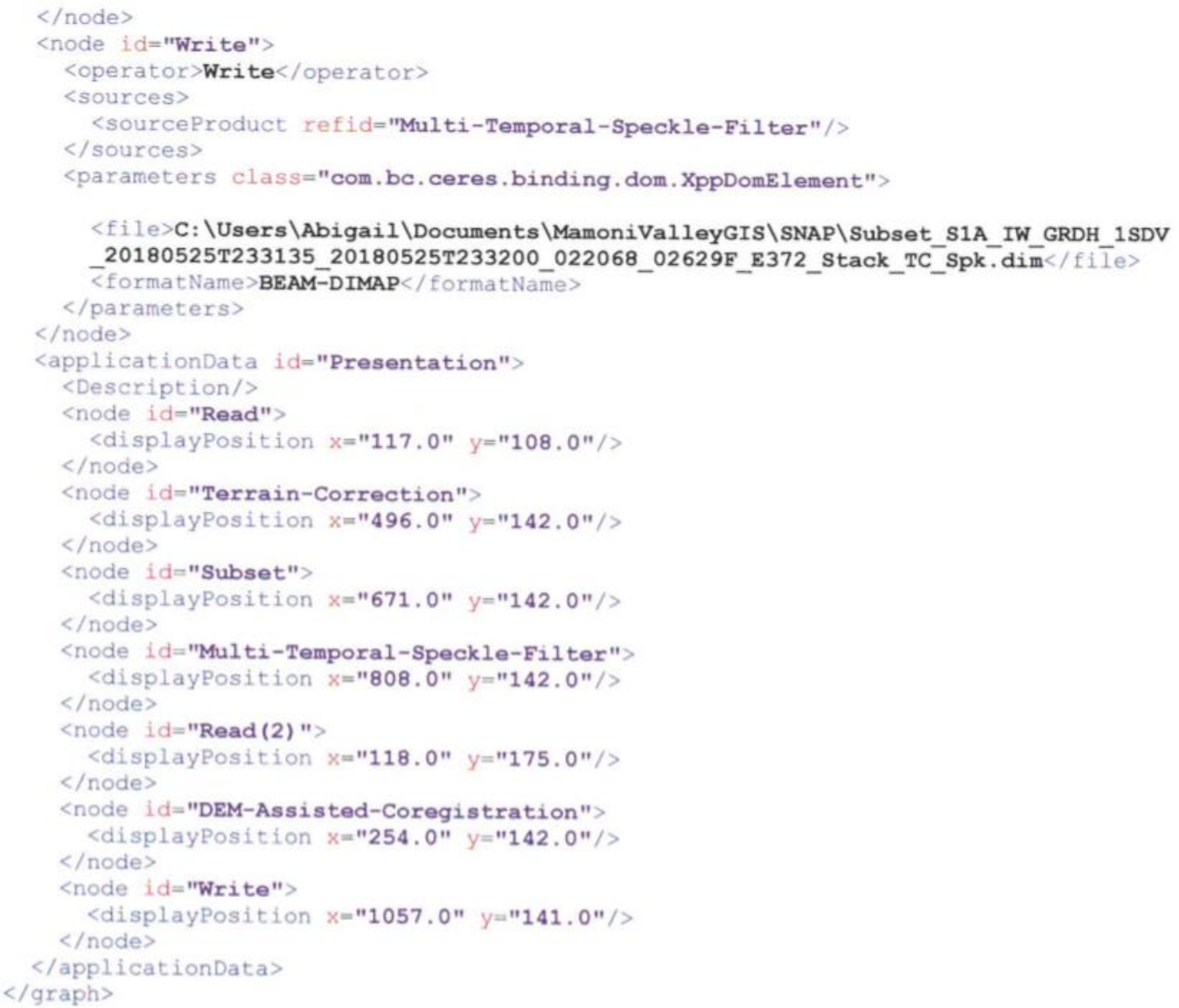

